\begin{tabular}{|c|l|}
\hline Title & A theory of Miyawaki liftings : the Hilbert-Siegel case \\
\hline Author(s) & A tobe, Hiraku \\
\hline Citation & $\begin{array}{l}\text { Mathematische annalen, 376/3-4), 1467-1535 } \\
\text { https://doi.org/40.1007/300208-019-01946-w }\end{array}$ \\
\hline Issue Date & 2020-04 \\
\hline Doc URL & http://hdl.handle.net/2115/80775 \\
\hline Rights & $\begin{array}{l}\text { This is a post-peer-review, pre copyedit version of an article published in Mathematische Annalen. The final } \\
\text { authenticated version is available online at: https:/doi.org/10.1007/s00208-019-01946-w }\end{array}$ \\
\hline Type & article (author version) \\
\hline File Information & Math. A nn. 376-3-4_1467-1535.pdf \\
\hline
\end{tabular}

Instructions for use 


\title{
A THEORY OF MIYAWAKI LIFTINGS: THE HILBERT-SIEGEL CASE
}

\author{
HIRAKU ATOBE
}

\begin{abstract}
The Miyawaki liftings are defined by the pullbacks of Ikeda liftings. Recently, Ikeda and Yamana extended the theory of Ikeda liftings to Hilbert-Siegel modular forms. In this paper, using their results, we establish a theory of Miyawaki liftings, both locally and globally. In the local theory, we describe the Miyawaki liftings for almost tempered unitary representations explicitly. In the global theory, we discuss the non-vanishing of the Miyawaki liftings using seesaw identities and the global Gan-Gross-Prasad conjecture. As an application of local Miyawaki liftings, we prove a new case of the local Gan-Gross-Prasad conjecture.
\end{abstract}

\section{CONTENTS}

1. Introduction

2. Ikeda liftings and their Fourier-Jacobi coefficients $\quad 7$

3. Local Miyawaki liftings $\quad 17$

4. Global Miyawaki liftings 31

5. A relation between Miyawaki liftings and the Gan-Gross-Prasad conjecture 37

Appendix A. Jacquet modules of representations of metaplectic groups 40

Appendix B. Local Langlands correspondence and Arthur's multiplicity formula $\quad 42$

Appendix C. Gan-Gross-Prasad conjectures 49

References $\quad 52$

\section{INTRODUCTION}

In 1992, Miyawaki [39] predicted the existence of certain Siegel modular forms. Let $S_{k}\left(\operatorname{Sp}_{n}(\mathbb{Z})\right)$ be the space of Siegel cusp forms of degree $n$, weight $k$, and level one.

Conjecture 1.1 (Miyawaki [39]). For normalized Hecke eigenforms $f \in S_{2 k-4}\left(\mathrm{SL}_{2}(\mathbb{Z})\right)$ and $g \in$ $S_{k}\left(\mathrm{SL}_{2}(\mathbb{Z})\right)$, there should exist a Hecke eigenform $F_{f, g} \in S_{k}\left(\operatorname{Sp}_{3}(\mathbb{Z})\right)$ whose standard L-function is given by

$$
L\left(s, F_{f, g}, \mathrm{st}\right)=L(s, g, \mathrm{Ad}) L(s+k-2, f) L(s+k-3, f) .
$$

In 2006, to approach Miyawaki's conjecture, Ikeda [28] constructed certain liftings, which are now called the Miyawaki liftings, as follows: For positive even $k$, a normalized Hecke eigenform $f \in S_{2 k-2(n+r)}\left(\mathrm{SL}_{2}(\mathbb{Z})\right)$ gives the Ikeda lift $F^{(2 n+2 r)} \in S_{k}\left(\operatorname{Sp}_{2 n+2 r}(\mathbb{Z})\right)$ (defined up to a constant). For the (classical) Ikeda lifting, see [27]. For a Hecke eigenform $g \in S_{k}\left(\operatorname{Sp}_{r}(\mathbb{Z})\right)$, Ikeda [28] defined the Miyawaki lift $\mathcal{M}^{(2 n+r)}\left(g, F^{(2 n+2 r)}\right)$ by the integral

$$
\mathcal{M}^{(2 n+r)}\left(g, F^{(2 n+2 r)}\right)\left(Z_{2 n+r}\right)
$$

2010 Mathematics Subject Classification. Primary 11F70; Secondary 11F46.

Key words and phrases. Automorphic representations; Miyawaki liftings; Gan-Gross-Prasad conjecture. 


$$
=\int_{\mathrm{Sp}_{r}(\mathbb{Z}) \backslash \mathfrak{H}_{r}} F^{(2 n+2 r)}\left(\left(\begin{array}{cc}
Z_{2 n+r} & 0 \\
0 & Z_{r}
\end{array}\right)\right) \overline{g^{c}\left(Z_{r}\right)}\left(\operatorname{det} \operatorname{Im} Z_{r}\right)^{k-r-1} d Z_{r},
$$

where $\mathfrak{H}_{r}$ is the Siegel upper half space of genus $r$ and we set $g^{c}\left(Z_{r}\right)=\overline{g\left(-\overline{Z_{r}}\right)}$. It is easy to see that $\mathcal{M}^{(2 n+r)}\left(g, F^{(2 n+2 r)}\right) \in S_{k}\left(\operatorname{Sp}_{2 n+r}(\mathbb{Z})\right)$. Ikeda proved the following:

Theorem 1.2 (Ikeda [28, Theorem 1.1]). If $\mathcal{M}^{(2 n+r)}\left(g, F^{(2 n+2 r)}\right)$ is not identically zero, then it is a Hecke eigenform with standard L-function

$$
L\left(s, \mathcal{M}^{(2 n+r)}\left(g, F^{(2 n+2 r)}\right), \mathrm{st}\right)=L(s, g, \mathrm{st}) \prod_{i=1}^{2 n} L(s+k-r-i, f) .
$$

Therefore Miyawaki's conjecture (Conjecture 1.1) was reduced to the non-vanishing of $\mathcal{M}^{(3)}\left(g, F^{(4)}\right)$. Ikeda gave a conjectural formula for the Petersson norm of $\mathcal{M}^{(2 n+r)}\left(g, F^{(2 n+2 r)}\right)$, and proposed the following conjecture.

Conjecture 1.3 (Ikeda [28, Conjecture 5.1]). (1) When $n=0$, the Miyawaki lift $\mathcal{M}^{(r)}\left(g, F^{(2 r)}\right)$ is nonzero if and only if the central value of the tensor product $L$-function $L(s, \operatorname{st}(g) \otimes f)$ is nonzero.

(2) When $n>0$, the Miyawaki lift $\mathcal{M}^{(2 n+r)}\left(g, F^{(2 n+2 r)}\right)$ is always nonzero.

Ichino [25] and Xue [57] proved Conjecture 1.3 (1) for the case where $r=1$ independently. Garrett-Heim [17] established a Hecke duality of Ikeda liftings and gave a preliminary answer to Conjecture $1.3(1)$.

Nowadays Miyawaki's conjecture (Conjecture 1.1) follows from Arthur's multiplicity formula ([3, Theorem 1.5.2]), which was established in 2013. However, this formula tells us only the existence of modular forms (or automorphic representations). The non-vanishing of Miyawaki liftings (Conjecture 1.3) is still open and interesting. The integral representations of Miyawaki liftings would imply several properties, which do not follow from Arthur's multiplicity formula.

Recently, Ikeda-Yamana [29] extended the theory of Ikeda liftings to Hilbert-Siegel modular forms using representation theory. The purpose of this paper is to establish a theory of Miyawaki liftings using the extended Ikeda liftings. We will define the Miyawaki liftings more generally, and give their several properties. Moreover, we will approach the non-vanishing problem of Miyawaki liftings using the Gan-Gross-Prasad conjecture.

To describe our results, let $F$ be a totally real number field, and $\mathbb{A}$ be the ring of adeles of $F$. The ring of finite adeles of $F$ is denoted by $\mathbb{A}_{\text {fin }}$. For a place $v$ of $F$, we write $v<\infty$ (resp. $v \mid \infty$ ) if $v$ is a finite place (resp. if $v$ is an infinite place). Fix a non-trivial unitary character $\psi$ of $\mathbb{A} / F$ such that for $v \mid \infty$, the local component $\psi_{v}$ is of the form $\psi_{v}\left(x_{v}\right)=\exp \left(2 \pi a_{v} \sqrt{-1} x_{v}\right)$ for $x_{v} \in F_{v} \cong \mathbb{R}$ with fixed $a_{v}>0$. Let $\tau=\otimes_{v}^{\prime} \tau_{v}$ be an irreducible cuspidal automorphic representation of $\mathrm{PGL}_{2}(\mathbb{A})$ satisfying the following conditions:

(A1) For $v<\infty, \tau_{v}$ is a principal series $\mu_{v} \times \mu_{v}^{-1}:=\operatorname{Ind}_{P_{(1,1)}\left(F_{v}\right)}^{\mathrm{GL}_{2}\left(F_{v}\right)}\left(\mu_{v} \otimes \mu_{v}^{-1}\right)$, where $P_{(1,1)}$ is the Borel subgroup of $\mathrm{GL}_{2}$ consisting of upper triangular matrices. (Note that $\mu_{v} \times \mu_{v}^{-1}$ has the trivial central character so that $\mathrm{PGL}_{2}\left(F_{v}\right)$ acts on it.)

(A2) For $v \mid \infty, \tau_{v}$ is a discrete series representation with lowest weight $\pm 2 k_{v}$, where $k_{v}>0$.

(A3) The root number $\varepsilon(1 / 2, \tau)$ is equal to 1 .

Let $\mathrm{Sp}_{n}$ be the symplectic group of rank $n$. For each place $v$, we denote the metaplectic double cover of $\operatorname{Sp}_{n}\left(F_{v}\right)$ by $\widetilde{\operatorname{Sp}_{n}}\left(F_{v}\right)$. Identify $\widetilde{\operatorname{Sp}_{n}}\left(F_{v}\right)=\operatorname{Sp}_{n}\left(F_{v}\right) \times\{ \pm 1\}$ as sets. Let $P_{n}$ be the Siegel 
parabolic subgroup of $\mathrm{Sp}_{n}$, and $\widetilde{P}_{n}\left(F_{v}\right)$ be the inverse image of $P_{n}(F)$ in $\widetilde{\operatorname{Sp}}_{n}\left(F_{v}\right)$. When $v<\infty$, we set

$$
I_{\psi_{v}}^{(n)}\left(\tau_{v}\right)=\operatorname{Ind}_{\widetilde{P}_{n}\left(F_{v}\right)}^{\widetilde{\mathrm{Sp}}_{n}\left(F_{v}\right)}\left(\mu_{v}^{(n)}\right)
$$

to be a degenerate principal series. Here,

$$
\mu_{v}^{(n)}\left(\left(\begin{array}{cc}
A & 0 \\
0 & { }^{t} A^{-1}
\end{array}\right), \zeta\right)=\zeta^{n}\left(\frac{\alpha_{\psi_{v}}(1)}{\alpha_{\psi_{v}}(\operatorname{det} A)}\right)^{n} \mu_{v}(\operatorname{det} A)
$$

for $A \in \mathrm{GL}_{n}\left(F_{v}\right)$ and $\zeta \in\{ \pm 1\}$, where $\alpha_{\psi_{v}}(a)$ is the Weil constant (see $\left.\S 2.2\right)$. It is known that $I_{\psi_{v}}^{(n)}\left(\tau_{v}\right)$ is irreducible $([35,50])$. Set $k+(n / 2)=\left(k_{v}+(n / 2)\right)_{v} \in \prod_{v \mid \infty} \mathbb{Z}$. Let $\widetilde{\operatorname{Sp}_{n}}(\mathbb{A})$ be the metaplectic double cover of the adelic group $\operatorname{Sp}_{n}(\mathbb{A})$. We denote the set of holomorphic cusp forms of weight $k+(n / 2)$ by $\mathcal{S}_{k+(n / 2)}\left(\operatorname{Sp}_{n}(F) \backslash \widetilde{\operatorname{Sp}}_{n}(\mathbb{A})\right)$. For the definition, see $\S 2.5$. IkedaYamana [29] showed that the irreducible representation $I_{\psi}^{(n)}(\tau)=\otimes_{v<\infty}^{\prime} I_{\psi_{v}}^{(n)}\left(\tau_{v}\right)$ of $\widetilde{\mathrm{Sp}_{n}}\left(\mathbb{A}_{\text {fin }}\right)$ appears in $\mathcal{S}_{k+(n / 2)}\left(\operatorname{Sp}_{n}(F) \backslash \widetilde{\operatorname{Sp}}_{n}(\mathbb{A})\right)$ with multiplicity one. We denote the unique subrepresentation of $\mathcal{S}_{k+(n / 2)}\left(\operatorname{Sp}_{n}(F) \backslash \widetilde{\operatorname{Sp}}_{n}(\mathbb{A})\right)$ which is isomorphic to $I_{\psi}^{(n)}(\tau)$ by $\operatorname{Ik}_{\psi}^{(n)}(\tau)$, and call it the Ikeda lift of $\tau$. by

Now let $n, r$ be non-negative integers. Then we have an embedding $\iota: \operatorname{Sp}_{n} \times \operatorname{Sp}_{r} \rightarrow \operatorname{Sp}_{n+r}$ given

$$
\iota\left(\left(\begin{array}{ll}
A_{1} & B_{1} \\
C_{1} & D_{1}
\end{array}\right),\left(\begin{array}{ll}
A_{2} & B_{2} \\
C_{2} & D_{2}
\end{array}\right)\right)=\left(\begin{array}{cc|cc}
A_{1} & 0 & B_{1} & 0 \\
0 & A_{2} & 0 & B_{2} \\
\hline C_{1} & 0 & D_{1} & 0 \\
0 & C_{2} & 0 & D_{2}
\end{array}\right) .
$$

For an admissible (not necessarily irreducible) representation $\pi$ of $\widetilde{\operatorname{Sp}_{r}}\left(\mathbb{A}_{\text {fin }}\right)$ occurring in the space $\mathcal{S}_{k+(n+r) / 2}\left(\operatorname{Sp}_{r}(F) \backslash \widetilde{\operatorname{Sp}_{r}}(\mathbb{A})\right)$, we define the (global) Miyawaki lift $\mathcal{M}_{\psi, \tau}^{(n)}(\pi)$ of $\pi$ by the representation of $\widetilde{\mathrm{Sp}}_{n}\left(\mathbb{A}_{\mathrm{fin}}\right)$ generated by the integrals

$$
\mathcal{M}^{(n)}\left(\left(g_{n}, \zeta_{n}\right) ; \varphi, \mathcal{F}\right)=\int_{\operatorname{Sp}_{r}(F) \backslash \operatorname{Sp}_{r}(\mathbb{A})} \mathcal{F}\left(\iota\left(g_{n}, g_{r}\right), \zeta_{n} \zeta_{r}\right) \overline{\varphi\left(g_{r}, \zeta_{r}\right)} d g_{r}
$$

for $\varphi \in \pi, \mathcal{F} \in \operatorname{Ik}_{\psi}^{(n+r)}(\tau)$ and $\left(g_{n}, \zeta_{n}\right) \in \widetilde{\operatorname{Sp}}_{n}(\mathbb{A})$. This is a subrepresentation of $\mathcal{S}_{k+(n+r) / 2}\left(\operatorname{Sp}_{n}(F) \backslash \widetilde{\operatorname{Sp}_{n}}(\mathbb{A})\right)$. We summarize several properties of Miyawaki liftings.

Theorem 1.4. Let $\pi$ be an irreducible representation of $\widetilde{\operatorname{Sp}_{r}}\left(\mathbb{A}_{\text {fin }}\right)$ occurring in $\mathcal{S}_{k+(n+r) / 2}\left(\operatorname{Sp}_{r}(F) \backslash \widetilde{\operatorname{Sp}_{r}}(\mathbb{A})\right)$, and $\mathcal{M}_{\psi, \tau}^{(n)}(\pi)$ be its Miyawaki lift. Suppose that $\mathcal{M}_{\psi, \tau}^{(n)}(\pi) \neq 0$ and $n \geq r$.

(1) If $\pi$ has an A-parameter $\Psi$, then $\mathcal{M}_{\psi, \tau}^{(n)}(\pi)$ has an A-parameter

$$
\Psi \boxplus \tau \chi_{-1}^{[(n+r) / 2]}[n-r],
$$

where $\chi_{-1}$ is the quadratic character of $\mathbb{A}^{\times} / F^{\times}$corresponding to $F(\sqrt{-1}) / F$. For the notation of A-parameters, see §4.2, or Appendix B.4 for a more detailed explanation.

(2) Suppose that $\pi$ has a tempered A-parameter. Then $\mathcal{M}_{\psi, \tau}^{(n)}(\pi)$ is irreducible, and $\mathcal{M}_{\psi, \tau}^{(n)}(\pi) \cong$ $\otimes_{v<\infty}^{\prime} \mathcal{M}_{\psi_{v}, \tau_{v}}^{(n)}\left(\pi_{v}\right)$. Here, $\mathcal{M}_{\psi_{v}, \tau_{v}}^{(n)}\left(\pi_{v}\right)$ is the local Miyawaki lift of $\pi_{v}$ described below.

(3) We have

$$
\pi \subset \mathcal{M}_{\psi, \tau}^{(r)}\left(\mathcal{M}_{\psi, \tau}^{(n)}(\pi)\right)
$$


If $\pi$ has a tempered A-parameter and if $r \leq n \leq r+1$ or $n>2 r$, then the inclusion is in fact an equality.

The statements in Theorem 1.4 are proven in Proposition 4.3, Proposition 4.4, and Theorem 4.5. Remark that Arthur's multiplicity formula ([3, Theorem 1.5.2]) should imply the existence of an irreducible subrepresentation of $\mathcal{S}_{k+(n+r) / 2}\left(\operatorname{Sp}_{r}(F) \backslash \operatorname{Sp}_{r}(\mathbb{A})\right)$ satisfying Theorem 1.4 (1) at least when $n+r$ is even.

The definition of Miyawaki liftings is similar to the one of theta liftings. As Miyawaki liftings are given by the pullbacks of Ikeda liftings, theta liftings are defined by the pullbacks of theta functions. One of the most important properties of theta liftings is the seesaw identities, which have several applications. For instance, by using seesaw identities, Ichino [25] computed the pullbacks of Saito-Kurokawa liftings, which is regarded as a special case of Ikeda's conjectural formula for the Petersson norm of a certain Miyawaki lift. For another example, Xue [56] reduced the refined version of the Gan-Gross-Prasad conjecture for the symplectic-metaplectic case to the one for the special orthogonal case.

Miyawaki liftings also satisfy certain seesaw identities. To state these identities, we introduce the Fourier-Jacobi periods. Fix a totally positive element $\xi \in F^{\times}$and set $\psi_{\xi}(x)=\psi(\xi x)$ for $x \in \mathbb{A}$. Let $n^{\prime}=n$ or $n^{\prime}=n-1$. For $\varphi \in \mathcal{S}_{l / 2}\left(\operatorname{Sp}_{n}(F) \backslash \widetilde{\operatorname{Sp}_{n}}(\mathbb{A})\right), \varphi^{\prime} \in \mathcal{S}_{l^{\prime} / 2}\left(\operatorname{Sp}_{n^{\prime}}(F) \backslash{\widetilde{\operatorname{Sp}_{n^{\prime}}}}(\mathbb{A})\right)$, and $\phi \in \mathcal{S}\left(\mathbb{A}^{n^{\prime}}\right)$, we define the Fourier-Jacobi period $\mathcal{P}_{n, n^{\prime}, \psi_{\xi}}\left(\varphi, \overline{\varphi^{\prime}}, \phi\right)$ by the integral

$$
\begin{cases}\int_{\operatorname{Sp}_{n}(F) \backslash \operatorname{Sp}_{n}(\mathbb{A})} \varphi(g, \zeta) \overline{\varphi^{\prime}(g, \zeta)} \overline{\Theta_{\psi_{\xi}}^{\phi}(g, \zeta)} d g, & \text { if } n^{\prime}=n, \\ \int_{V_{n-1}(F) \backslash V_{n-1}(\mathbb{A})} \int_{\operatorname{Sp}_{n-1}(F) \backslash \operatorname{Sp}_{n-1}(\mathbb{A})} \varphi(v(g, \zeta)) \overline{\varphi^{\prime}(g, \zeta)} \overline{\Theta_{\psi_{\xi}}^{\phi}(v(g, \zeta))} d g d v, & \text { if } n^{\prime}=n-1 .\end{cases}
$$

Here, $\Theta_{\psi_{\xi}}^{\phi}$ is the theta function associated to $\phi \in \mathcal{S}\left(\mathbb{A}^{n^{\prime}}\right)$, which is a genuine automorphic form on a Jacobi group $\widetilde{J}_{n^{\prime}}(\mathbb{A})={\widetilde{\mathrm{Sp}_{n^{\prime}}}}(\mathbb{A}) \ltimes V_{n^{\prime}}(\mathbb{A})$, where $V_{n^{\prime}} \cong F^{2 n^{\prime}} \oplus F$ is a Heisenberg group. Let $\mathcal{S}\left(\mathbb{A}^{n^{\prime}}\right)_{\xi}$ be the subspace of $\mathcal{S}\left(\mathbb{A}^{n^{\prime}}\right)$ consisting of lowest weight vectors of the Weil representation of $\widetilde{J}_{n^{\prime}}(\mathbb{A})$ with respect to $\psi_{\xi}$.

Proposition 1.5 (Seesaw identity (Proposition 4.7)). Let $\pi$ and $\pi^{\prime}$ be irreducible representations of $\widetilde{\operatorname{Sp}_{r}}\left(\mathbb{A}_{\text {fin }}\right)$ and $\widetilde{\operatorname{Sp}_{n-1}}\left(\mathbb{A}_{\text {fin }}\right)$ occurring in $\mathcal{S}_{k+(n+r) / 2}\left(\operatorname{Sp}_{r}(F) \backslash \widetilde{\operatorname{Sp}_{r}}(\mathbb{A})\right)$ and $\mathcal{S}_{k+(n-1+r) / 2}\left(\operatorname{Sp}_{n-1}(F) \backslash \widetilde{\operatorname{Sp}_{n-1}}(\mathbb{A})\right)$, respectively.

(1) If there exist $\mathcal{M}^{(n)}\left(\varphi, \mathcal{F}_{1}\right) \in \mathcal{M}_{\psi, \tau}^{(n)}(\pi)$ with $\varphi \in \pi, \varphi^{\prime} \in \pi^{\prime}$, and $\phi_{1} \in \mathcal{S}\left(\mathbb{A}^{n-1}\right)_{\xi}$ such that

$$
\mathcal{P}_{n, n-1, \psi_{\xi}}\left(\mathcal{M}^{(n)}\left(\varphi, \mathcal{F}_{1}\right), \overline{\varphi^{\prime}}, \phi_{1}\right) \neq 0,
$$

then there exist $\mathcal{M}^{(r)}\left(\varphi^{\prime}, \mathcal{F}_{2}^{\prime}\right) \in \mathcal{M}_{\psi, \tau \chi_{\xi}}^{(r)}\left(\pi^{\prime}\right)$ and $\phi_{2} \in \mathcal{S}\left(\mathbb{A}^{r}\right)_{\xi}$ such that

$$
\mathcal{P}_{r, r, \psi_{\xi}}\left(\varphi, \overline{\mathcal{M}^{(r)}\left(\varphi^{\prime}, \mathcal{F}_{2}^{\prime}\right)}, \phi_{2}\right) \neq 0
$$

(2) Assume that $n+r \geq 2$. If there exist $\varphi \in \pi, \mathcal{M}^{(r)}\left(\varphi^{\prime}, \mathcal{F}_{2}^{\prime}\right) \in \mathcal{M}_{\psi, \tau \chi \xi}^{(r)}\left(\pi^{\prime}\right)$ with $\varphi^{\prime} \in \pi^{\prime}$, and $\phi_{2} \in \mathcal{S}\left(\mathbb{A}^{r}\right)_{\xi}$ such that

$$
\mathcal{P}_{r, r, \psi_{\xi}}\left(\varphi, \overline{\mathcal{M}^{(r)}\left(\varphi^{\prime}, \mathcal{F}_{2}^{\prime}\right)}, \phi_{2}\right) \neq 0
$$


then there exist $\mathcal{M}^{(n)}\left(\varphi, \mathcal{F}_{1}\right) \in \mathcal{M}_{\psi, \tau}^{(n)}(\pi)$ and $\phi_{1} \in \mathcal{S}\left(\mathbb{A}^{n-1}\right)_{\xi}$ such that

$$
\mathcal{P}_{n, n-1, \psi_{\xi}}\left(\mathcal{M}^{(n)}\left(\varphi, \mathcal{F}_{1}\right), \overline{\varphi^{\prime}}, \phi_{1}\right) \neq 0 .
$$

We shall write these properties as the following seesaw diagram:

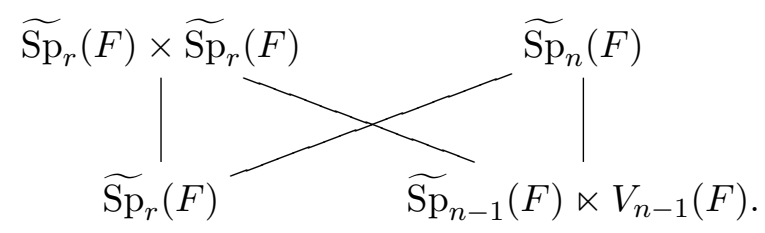

Remark that the proof of Proposition 1.5 uses the non-vanishing of Fourier-Jacobi coefficients of Ikeda liftings (Proposition $2.7(3)$ ). In particular, the seesaw identities would not follow from Arthur's multiplicity formula.

Finally, following Ikeda's conjecture (Conjecture 1.3), we formulate a conjecture on the nonvanishing of Miyawaki liftings.

Conjecture 1.6 (Conjecture 5.1). Let $\pi$ be an irreducible representation of $\widetilde{\operatorname{Sp}_{r}}\left(\mathbb{A}_{\text {fin }}\right)$ occurring in $\mathcal{S}_{k+(n+r) / 2}\left(\operatorname{Sp}_{r}(F) \backslash \widetilde{\operatorname{Sp}}_{r}(\mathbb{A})\right)$.

(1) When $n=r$, the Miyawaki lift $\mathcal{M}_{\psi, \tau}^{(r)}(\pi)$ is nonzero if and only if the central value of the Rankin-Selberg L-function $L\left(s, \pi \times \tau \chi_{-1}^{r}\right)$ is nonzero.

(2) When $n>r$, the Miyawaki lift $\mathcal{M}_{\psi, \tau}^{(n)}(\pi)$ is always nonzero.

Using the seesaw identities (Proposition 1.5), one can prove the easiest case.

Proposition 1.7 (Corollary 5.6). Suppose that $n=r=1$. Let $\pi$ be an irreducible representation of $\mathrm{SL}_{2}\left(\mathbb{A}_{\text {fin }}\right)$ occurring in $\mathcal{S}_{k+1}\left(\mathrm{SL}_{2}(F) \backslash \mathrm{SL}_{2}(\mathbb{A})\right)$. Then $\mathcal{M}_{\psi, \tau}^{(1)}(\pi) \neq 0$ if and only if $L(1 / 2, \pi \times \tau \chi-1) \neq$ 0 .

Outline of the proof. The Miyawaki lift $\mathcal{M}_{\psi, \tau}^{(1)}(\pi)$ is nonzero if and only if there exists a vector in $\mathcal{M}_{\psi, \tau}^{(1)}(\pi)$ whose $\xi$-th Fourier coefficient is nonzero for some totally positive element $\xi \in F^{\times}$. This condition means that $\mathcal{P}_{1,0, \psi_{\xi}}$ is nonzero on $\mathcal{M}_{\psi, \tau}^{(1)}(\pi) \times \bar{\eta} \times \mathcal{S}\left(\mathbb{A}^{0}\right)_{\xi}$, where $\eta$ is the nontrivial character of $\widetilde{\mathrm{Sp}}_{0}(\mathbb{A})=\{ \pm 1\}$. By the seesaw identity (Proposition 1.5), it is equivalent that $\mathcal{P}_{1,1, \psi_{\xi}}$ is nonzero on $\pi \times \overline{\mathcal{M}_{\psi, \tau \chi_{\xi}}^{(1)}(\eta)} \times \mathcal{S}\left(\mathbb{A}^{1}\right)_{\xi}$. Note that $\mathcal{M}_{\psi, \tau \chi \xi}^{(1)}(\eta)$ is the Ikeda lift $I_{\psi}^{(1)}\left(\tau \chi_{\xi}\right)$, i.e., the Shimura correspondence of $\tau \chi_{\xi}$. By the global Gan-Gross-Prasad conjecture for $\mathrm{SL}_{2} \times \widetilde{\mathrm{SL}}_{2}$ proven by GanGurevich [12], Qiu [44], and Xue [56], the non-vanishing of the Fourier-Jacobi period $\mathcal{P}_{1,1, \psi_{\xi}}$ on $\pi \times \overline{I_{\psi}^{(1)}\left(\tau \chi_{\xi}\right)} \times \mathcal{S}\left(\mathbb{A}^{1}\right)_{\xi}$ is equivalent to the non-vanishing of the central value of $L(s, \pi \times \tau \chi-1)$.

By a similar argument, we can relate Conjecture 1.6 to the Gan-Gross-Prasad conjecture (see Conjecture 5.2 below). The following theorem summarizes Theorems 5.5 and 5.7.

Theorem 1.8. (1) Assume the Gan-Gross-Prasad conjecture (Conjecture 5.2) and Hypothesis 5.3 (A) below. Then Conjecture 1.6 for $n=r, r+1$ holds when $\pi$ has a tempered $A$ parameter.

(2) Assume Hypothesis $5.3(B)$ or $(C)$ below. Then Conjecture 1.6 for $n>r$ is reduced to the case where $n-r=1$. 
Hypothesis 5.3 is an expectation of the existence of certain (tempered) automorphic representations. This was also considered in the theory of twisted automorphic descents introduced by Jiang-Zhang [32, Conjecture 2.3]. For more comments, see Remark 5.4 below.

There is a local analogue of Miyawaki liftings. Now let $F$ be a non-archimedean local field of characteristic zero, and $\psi$ be a non-trivial additive character of $F$. Let $\chi_{-1}$ be the quadratic character of $F^{\times}$corresponding to $F(\sqrt{-1}) / F$. A local analogue of the Ikeda lifting is the degenerate principal series $I_{\psi}^{(n+r)}(\tau)=\operatorname{Ind}_{\widetilde{P}_{n+r}(F)} \widetilde{\mathrm{Sp}}_{n+r}(F)\left(\mu^{(n+r)}\right)$, where $\tau=\mu \times \mu^{-1}$ is a principal series of $\mathrm{GL}_{2}(F)$ with $\mu$ being a unitary character of $F^{\times}$. Recall that there is an embedding $\iota: \operatorname{Sp}_{n} \times \operatorname{Sp}_{r} \hookrightarrow \operatorname{Sp}_{n+r}$. For an irreducible representation $\pi$ of $\widetilde{\mathrm{Sp}_{r}}(F)$, on which the kernel $\{ \pm 1\}$ of the covering map $\widetilde{\operatorname{Sp}}_{r}(F) \rightarrow \operatorname{Sp}_{r}(F)$ acts by $( \pm 1)^{n+r}$, the maximal $\pi$-isotypic quotient of $I_{\psi}^{(n+r)}(\tau)$ is of the form

$$
\mathcal{M}_{\psi, \tau}^{(n)}(\pi) \otimes \pi
$$

for some smooth representation $\mathcal{M}_{\psi, \tau}^{(n)}(\pi)$ of $\widetilde{\operatorname{Sp}}_{n}(F)$, on which the kernel $\{ \pm 1\}$ of the covering map $\widetilde{\operatorname{Sp}_{n}}(F) \rightarrow \operatorname{Sp}_{n}(F)$ acts by $( \pm 1)^{n+r}$. We call $\mathcal{M}_{\psi, \tau}^{(n)}(\pi)$ the local Miyawaki lift of $\pi$. The following is the main local theorem.

Theorem 1.9 (Theorem 3.1). Let $\mu$ be a unitary character of $F^{\times}$, and $\pi$ be an irreducible representation of $\widetilde{\operatorname{Sp}}_{r}(F)$ on which $\{ \pm 1\}$ acts by $( \pm 1)^{n+r}$. Suppose that $n \geq r$.

(1) The local Miyawaki lift $\mathcal{M}_{\psi, \tau}^{(n)}(\pi)$ is nonzero and of finite length.

(2) If $\pi$ is almost tempered (see $§ 3.2$ below) and unitary, then

- $\mathcal{M}_{\psi, \tau}^{(n)}(\pi)$ is irreducible;

- $\mathcal{M}_{\psi, \tau}^{(n)}(\pi) \cong\left(\mu^{\prime} \circ \operatorname{det}_{n-r}\right) \rtimes \pi:=\operatorname{Ind}_{\widetilde{P}_{n-r}(F)}^{\widetilde{\mathrm{Sp}}_{n}(F)}\left(\left(\mu^{\prime} \circ \operatorname{det}_{n-r}\right) \otimes \pi\right)$ with $\mu^{\prime}=\mu \chi_{-1}^{[(n+r) / 2]}$;

- $\mathcal{M}_{\psi, \tau}^{(n)}(\pi)$ is isomorphic to the unique irreducible quotient of the induced representation

$$
\begin{cases}\tau^{\prime}|\cdot|^{\frac{n-r-1}{2}} \times \tau^{\prime}|\cdot|^{\frac{n-r-3}{2}} \times \cdots \times \tau^{\prime}|\cdot|^{\frac{1}{2}} \rtimes \pi & \text { if } n+r \equiv 0 \bmod 2, \\ \tau^{\prime}|\cdot|^{\frac{n-r-1}{2}} \times \tau^{\prime}|\cdot|^{\frac{n-r-3}{2}} \times \cdots \times \tau^{\prime}|\cdot|^{1} \times \mu^{\prime} \rtimes \pi & \text { if } n+r \equiv 1 \bmod 2,\end{cases}
$$

where $\tau^{\prime}=\tau \otimes \chi_{-1}^{[(n+r) / 2]}=\mu^{\prime} \times \mu^{\prime-1}$.

For the notation of parabolic inductions, see §3.2 below.

(3) For any irreducible almost tempered unitary representations $\pi_{1}$ and $\pi_{2}$, we have

$$
\mathcal{M}_{\psi, \tau}^{(n)}\left(\pi_{1}\right) \cong \mathcal{M}_{\psi, \tau}^{(n)}\left(\pi_{2}\right) \Longrightarrow \pi_{1} \cong \pi_{2} .
$$

(4) Assume one of the following:

- $\pi$ is almost tempered and unitary, and $r \leq n \leq r+1$ or $n>2 r$;

- $\pi$ is discrete series so that $\mathcal{M}_{\psi, \tau}^{(n)}(\pi)$ is irreducible. Set

$$
\pi^{\prime}=\mathcal{M}_{\psi, \tau}^{(r)}\left(\mathcal{M}_{\psi, \tau}^{(n)}(\pi)\right) .
$$

Then all irreducible subquotients of $\pi^{\prime}$ are isomorphic to $\pi$, and the maximal semisimple quotient of $\pi^{\prime}$ is irreducible.

(5) Suppose that $\mu$ is unramified, and set $\alpha=\left(\mu \chi_{-1}^{[(n+r) / 2]}\right)(\varpi)$, where $\varpi$ is a uniformizer of $F$. If $\pi$ is an irreducible unramified representation of $\widetilde{\operatorname{Sp}}_{r}(F)$ with the Satake parameter 
$\left\{\beta_{1}^{ \pm 1}, \ldots, \beta_{r}^{ \pm 1}\right\}$, then $\mathcal{M}_{\psi, \tau}^{(n)}(\pi)$ has a unique irreducible unramified quotient. Its Satake parameter is equal to

$$
\left\{\beta_{1}^{ \pm 1}, \ldots, \beta_{r}^{ \pm 1}\right\} \cup\left\{\alpha^{ \pm 1} q^{\frac{n-r-1}{2}}, \alpha^{ \pm 1} q^{\frac{n-r-3}{2}}, \ldots, \alpha^{ \pm 1} q^{-\frac{n-r-1}{2}}\right\}
$$

as multisets, where $q$ is the cardinality of the residue field of $F$.

There is also a local analogue of seesaw identities (Proposition 1.5). For $\xi \in F^{\times}$, we denote the Weil representations of the Jacobi group $\widetilde{J}_{n-1}(F)={\widetilde{\mathrm{Sp}_{n-1}}}_{n}(F) \ltimes V_{n-1}(F)$ and the metaplectic group $\widetilde{\mathrm{Sp}_{r}}(F)$ with respect to $\psi_{\xi}$ by $\omega_{\psi_{\xi}}^{(n-1)}$ and $\omega_{\psi_{\xi}}^{(r)}$, respectively.

Proposition 1.10 (Seesaw identity (Proposition 3.10)). Let $\pi$ and $\pi^{\prime}$ be irreducible representations of ${\widetilde{\mathrm{Sp}_{r}}}_{r}(F)$ and $\widetilde{\mathrm{Sp}_{n-1}}(F)$, on which $\{ \pm 1\}$ acts by $( \pm 1)^{r+n}$ and $( \pm 1)^{r+n-1}$, respectively. Then

$$
\operatorname{Hom}_{\widetilde{J}_{n-1}(F)}\left(\left.\mathcal{M}_{\psi, \tau}^{(n)}(\pi)\right|_{\widetilde{J}_{n-1}(F)}, \pi^{\prime} \otimes \omega_{\psi_{\xi}}^{(n-1)}\right) \cong \operatorname{Hom}_{\widetilde{\mathrm{Sp}}_{r}(F)}\left(\mathcal{M}_{\psi, \tau \chi_{\xi}}^{(r)}\left(\pi^{\prime}\right) \otimes \omega_{\psi_{\xi}}^{(r)}, \pi\right) .
$$

As an application, the seesaw identity gives a quite new example of the local Gan-Gross-Prasad conjecture in a non-generic case (Theorem C.5).

This paper is organized as follows. In $\S 2$, we recall the theory of Ikeda liftings extended by Ikeda-Yamana [29]. The local and global theories of Miyawaki liftings are explained in $\S 3$ and $\S 4$, respectively. In $\S 5$, we discuss Conjecture 1.6 and its relation with the Gan-Gross-Prasad conjecture. In Appendices A, B and C, we recall results on Jacquet modules of representations of metaplectic groups, the local and global Langlands program, and the Gan-Gross-Prasad conjecture, respectively.

Acknowledgments. The author is grateful to Shunsuke Yamana, Atsushi Ichino, and Tamotsu Ikeda for their helpful comments. Thanks are also due to the referees for helpful comments. This work was supported by the Foundation for Research Fellowships of Japan Society for the Promotion of Science for Young Scientists (PD) Grant 29-193.

\section{IKEDA LIFTINGS AND THEIR FouRIER-JACOBI COEFFICIENTS}

In this section, we recall the theory of Ikeda liftings along with [29].

2.1. Metaplectic group and its representations. Let $F$ be a totally real number field. The symplectic group $\mathrm{Sp}_{n}$ is an algebraic group defined over $F$ given by

$$
\operatorname{Sp}_{n}(F)=\left\{g \in \mathrm{GL}_{2 n}(F) \mid{ }^{t} g\left(\begin{array}{cc}
0 & -\mathbf{1}_{n} \\
\mathbf{1}_{n} & 0
\end{array}\right) g=\left(\begin{array}{cc}
0 & -\mathbf{1}_{n} \\
\mathbf{1}_{n} & 0
\end{array}\right)\right\} .
$$

The set of symmetric matrices of size $n$ with coefficients in $F$ is denoted by $\operatorname{Sym}_{n}(F)$. For $A \in$ $\mathrm{GL}_{n}(F)$ and $B \in \operatorname{Sym}_{n}(F)$, set

$$
\mathbf{m}(A)=\left(\begin{array}{cc}
A & 0 \\
0 & { }^{t} A^{-1}
\end{array}\right), \quad \mathbf{n}(B)=\left(\begin{array}{cc}
\mathbf{1}_{n} & B \\
0 & \mathbf{1}_{n}
\end{array}\right) \in \operatorname{Sp}_{n}(F)
$$

For each $k=1, \ldots, n$, we define a standard maximal parabolic subgroup $P_{k}(F)$ of $\operatorname{Sp}_{n}(F)$ by

$$
P_{k}(F)=\left\{\left(\begin{array}{cc|cc}
a & * & * & * \\
0 & A & * & B \\
\hline 0 & 0 & { }^{t} a^{-1} & 0 \\
0 & C & * & D
\end{array}\right) \mid a \in \mathrm{GL}_{k}(F),\left(\begin{array}{cc}
A & B \\
C & D
\end{array}\right) \in \operatorname{Sp}_{n-k}(F)\right\} .
$$


Let $P_{k}(F)=M_{k}(F) N_{k}(F)$ be the standard Levi decomposition, so that the Levi $M_{k}(F)$ is isomorphic to $\mathrm{GL}_{k}(F) \times \operatorname{Sp}_{n-k}(F)$. In particular, $P_{n}(F)=M_{n}(F) N_{n}(F)$ is the Siegel parabolic subgroup with $M_{n}(F)=\left\{\mathbf{m}(A) \mid A \in \mathrm{GL}_{n}(F)\right\}$ and $N_{n}(F)=\left\{\mathbf{n}(B) \mid B \in \operatorname{Sym}_{n}(F)\right\}$. Let $B_{n}=\cap_{k=1}^{n} P_{k}$ be a Borel subgroup of $\mathrm{Sp}_{n}$. A parabolic subgroup $P$ of $\mathrm{Sp}_{n}$ is called standard if $P$ contains $B_{n}$.

For each place $v$ of $F$, we denote by $\widetilde{\operatorname{Sp}_{n}}\left(F_{v}\right)$ the metaplectic group, i.e., the topological double cover of the symplectic group $\operatorname{Sp}_{n}\left(F_{v}\right)$. As sets, we identify $\widetilde{\operatorname{Sp}_{n}}\left(F_{v}\right)$ with $\operatorname{Sp}_{n}\left(F_{v}\right) \times\{ \pm 1\}$. Then the group law of $\widetilde{\operatorname{Sp}}_{n}\left(F_{v}\right)$ is given by

$$
\left(g_{1}, \zeta_{1}\right)\left(g_{2}, \zeta_{2}\right)=\left(g_{1} g_{2}, c_{v}\left(g_{1}, g_{2}\right) \zeta_{1} \zeta_{2}\right)
$$

for $g_{1}, g_{2} \in \operatorname{Sp}_{n}\left(F_{v}\right)$ and $\zeta_{1}, \zeta_{2} \in\{ \pm 1\}$, where $c_{v}\left(g_{1}, g_{2}\right)$ is Rao's 2-cocycle of $\operatorname{Sp}_{n}\left(F_{v}\right)$ with values in $\{ \pm 1\}$. The double cover $\widetilde{\operatorname{Sp}}_{n}\left(F_{v}\right) \rightarrow \operatorname{Sp}_{n}\left(F_{v}\right)$ splits over the subgroup $N_{n}\left(F_{v}\right)$ by $\mathbf{n}(B) \mapsto(\mathbf{n}(B), 1)$. If $F_{v}$ is a non-archimedean local field whose residue characteristic is not 2 , there is a unique splitting

$$
\operatorname{Sp}_{n}\left(\mathfrak{o}_{v}\right) \rightarrow \widetilde{\operatorname{Sp}_{n}}\left(F_{v}\right), g \mapsto(g, s(g)) .
$$

Here, we denote by $\mathfrak{o}_{v}$ the ring of integers of $F_{v}$. We identify $N_{n}\left(F_{v}\right)$ and $\operatorname{Sp}_{n}\left(\mathfrak{o}_{v}\right)$ with the images of these splittings. If $H$ is a subgroup of $\operatorname{Sp}_{n}(F)$, the inverse image of $H$ in $\widetilde{\operatorname{Sp}}_{n}(F)$ is denoted by $\widetilde{H}$.

Next, we define the global metaplectic group. We denote the adele ring of $F$ by $\mathbb{A}$. Let $\mathfrak{S}$ be a finite set of places of $F$, which contains all places above 2 and $\infty$. Put

$$
\operatorname{Sp}_{n}(\mathbb{A})_{\mathfrak{S}}=\prod_{v \in \mathfrak{S}} \operatorname{Sp}_{n}\left(F_{v}\right) \times \prod_{v \notin \mathfrak{S}} \operatorname{Sp}\left(\mathfrak{o}_{v}\right)
$$

Then the double cover $\widetilde{\operatorname{Sp}_{n}}(\mathbb{A})_{\mathfrak{S}} \rightarrow \operatorname{Sp}_{n}(\mathbb{A})_{\mathfrak{S}}$ is defined by the 2-cocycle $\prod_{v \in \mathfrak{S}} c_{v}\left(g_{1, v}, g_{2, v}\right)$. For $\mathfrak{S}_{1} \subset \mathfrak{S}_{2}$, there exists an embedding $\widetilde{\operatorname{Sp}}_{n}(\mathbb{A})_{\mathfrak{S}_{1}} \hookrightarrow \widetilde{\operatorname{Sp}}_{n}(\mathbb{A})_{\mathfrak{S}_{2}}$ given by

$$
\left(\left(g_{v}\right)_{v}, \zeta\right) \mapsto\left(\left(g_{v}\right)_{v}, \zeta \prod_{v \in \mathfrak{S}_{2} \backslash \mathfrak{S}_{1}} s_{v}\left(g_{v}\right)\right) .
$$

The global metaplectic group $\widetilde{\operatorname{Sp}_{n}}(\mathbb{A})$ is defined by the inductive limit

$$
\widetilde{\operatorname{Sp}}_{n}(\mathbb{A})=\underset{\mathfrak{S}}{\lim } \widetilde{\operatorname{Sp}}_{n}(\mathbb{A})_{\mathfrak{S}}
$$

where $\mathfrak{S}$ runs over all finite sets of places of $F$ containing all places above 2 and $\infty$. The covering $\widetilde{\operatorname{Sp}}_{n}(\mathbb{A}) \rightarrow \operatorname{Sp}_{n}(\mathbb{A})$ splits over $\operatorname{Sp}_{n}(F)$ uniquely. We identify $\operatorname{Sp}_{n}(F)$ with the image of the splitting.

2.2. Weil representations of Jacobi groups. We recall the Weil representation on a Jacobi group in the local setting. Let $F$ be a local field of characteristic zero. Fix a non-trivial unitary character $\psi$ of $F$. For $\xi \in F^{\times}$, we define a new non-trivial unitary character $\psi_{\xi}$ by

$$
\psi_{\xi}(x)=\psi(\xi x)
$$

for $x \in F$. Let $\langle$,$\rangle be the quadratic Hilbert symbol. For x, \xi \in F^{\times}$, we set $\chi_{\xi}(x)=\langle x, \xi\rangle$. For each Schwartz function $f \in \mathcal{S}(F)$, the Fourier transform $\hat{f}$ (with respect to $\psi_{\xi}$ ) is defined by

$$
\hat{f}(x)=\int_{F} f(y) \psi_{\xi}(x y) d y
$$


where $d y$ is the self-dual Haar measure on $F$ with respect to $\psi_{\xi}$. For $a \in F^{\times}$, there exists an 8-th root of unity $\alpha_{\psi_{\xi}}(a)$ such that

$$
\int_{F} f(x) \psi_{\xi}\left(a x^{2}\right) d x=\alpha_{\psi_{\xi}}(a)|2 a|^{-\frac{1}{2}} \int_{F} \hat{f}(x) \psi_{\xi}\left(-\frac{x^{2}}{4 a}\right) d x
$$

for any $f \in \mathcal{S}(F)$. The constant $\alpha_{\psi_{\xi}}(a)$ is called the Weil constant. It satisfies that $\alpha_{\psi_{\xi}}\left(a b^{2}\right)=$ $\alpha_{\psi_{\xi}}(a)$ and

$$
\frac{\alpha_{\psi_{\xi}}(a) \alpha_{\psi_{\xi}}(b)}{\alpha_{\psi_{\xi}}(1) \alpha_{\psi_{\xi}}(a b)}=\langle a, b\rangle
$$

for $a, b \in F^{\times}$. In particular,

$$
\left(\frac{\alpha_{\psi_{\xi}}(1)}{\alpha_{\psi_{\xi}}(a)}\right)^{2}=\chi_{-1}(a),
$$

where $\chi_{-1}=\langle\cdot,-1\rangle$ is the quadratic character associated to $F(\sqrt{-1}) / F$.

Put

$$
\mathbf{v}(x, y, z)=\left(\begin{array}{cc|cc}
1 & x & z & y \\
0 & \mathbf{1}_{n-1} & { }^{t} y & 0 \\
\hline 0 & 0 & 1 & 0 \\
0 & 0 & -{ }^{t} x & \mathbf{1}_{n-1}
\end{array}\right) \in \operatorname{Sp}_{n}(F),
$$

where $x, y \in F^{n-1}$ are row vectors and $z \in F$. We set

$$
\begin{aligned}
& V(F)=V_{n-1}(F)=\left\{\mathbf{v}(x, y, z) \mid x, y \in F^{n-1}, z \in F\right\}, \\
& X(F)=X_{n-1}(F)=\left\{\mathbf{v}(x, 0,0) \mid x \in F^{n-1}\right\}, \\
& Y(F)=Y_{n-1}(F)=\left\{\mathbf{v}(0, y, 0) \mid y \in F^{n-1}\right\}, \\
& Z(F)=Z_{n-1}(F)=\{\mathbf{v}(0,0, z) \mid z \in F\} .
\end{aligned}
$$

Note that $V(F)$ is a Heisenberg group. We regard $\widetilde{\operatorname{Sp}}_{n-1}(F)$ as a subgroup of $\widetilde{\mathrm{Sp}}_{n}(F)$ by the embedding

$$
\left(\left(\begin{array}{cc}
A & B \\
C & D
\end{array}\right), \zeta\right) \mapsto\left(\left(\begin{array}{cc|cc}
1 & 0 & 0 & 0 \\
0 & A & 0 & B \\
\hline 0 & 0 & 1 & 0 \\
0 & C & 0 & D
\end{array}\right), \zeta\right) .
$$

By the Stone-von Neumann theorem, there is a unique irreducible admissible representation $\omega_{\psi_{\xi}}$ of $V(F)$ on which the center $Z(F)$ acts by $\psi_{\xi}$. This representation $\omega_{\psi_{\xi}}$ extends to the Weil representation of the group $\widetilde{J}_{n-1}(F)=V(F) \rtimes \widetilde{\mathrm{Sp}}_{n-1}(F)$. We call $J_{n-1}(F)=V(F) \rtimes \operatorname{Sp}_{n-1}(F)$ a Jacobi group. The representation $\omega_{\psi_{\xi}}$ is realized on the Schwartz space $\mathcal{S}(X(F))$ explicitly as follows:

$$
\begin{aligned}
\omega_{\psi_{\xi}}(\mathbf{v}(x, y, z)) \phi(t) & =\psi_{\xi}\left(z+2 t \cdot{ }^{t} y+x \cdot{ }^{t} y\right) \phi(t+x), \\
\omega_{\psi_{\xi}}(\mathbf{m}(A), \zeta) \phi(t) & =\zeta \frac{\alpha_{\psi_{\xi}}(1)}{\alpha_{\psi_{\xi}}(\operatorname{det} A)}|\operatorname{det} A|^{\frac{1}{2}} \phi(t A), \\
\omega_{\psi_{\xi}}(\mathbf{n}(B), \zeta) \phi(t) & =\zeta \psi_{\xi}\left(t \cdot B \cdot{ }^{t} t\right) \phi(t), \\
\omega_{\psi_{\xi}}\left(\left(\begin{array}{cc}
0 & -\mathbf{1}_{n-1} \\
\mathbf{1}_{n-1} & 0
\end{array}\right), \zeta\right) \phi(t) & =\zeta \alpha_{\psi_{\xi}}(1)^{-n+1}|2|^{\frac{n-1}{2}} \int_{X(F)} \phi(u) \overline{\psi_{\xi}\left(2 t \cdot{ }^{t} u\right)} d u
\end{aligned}
$$


for $\zeta \in\{ \pm 1\}, \mathbf{v}(x, y, z) \in V(F), A \in \mathrm{GL}_{n-1}(F), B \in \operatorname{Sym}_{n-1}(F)$, and $\phi \in \mathcal{S}(X(F))$. Here, $d u=\prod_{i} d u_{i}$ is the Haar measure on $X(F)$ with $d u_{i}$ being the self-dual Haar measure on $F$ with respect to $\psi_{\xi}$. The Weil representation $\omega_{\psi_{\xi}}$ is unitary with respect to the inner product

$$
\left(\phi_{1}, \phi_{2}\right)=\int_{X(F)} \phi_{1}(t) \overline{\phi_{2}(t)} d t
$$

for $\phi_{1}, \phi_{2} \in \mathcal{S}(X(F))$.

2.3. Non-archimedean case. In this subsection, we assume that $F$ is non-archimedean. For a smooth representation $\Pi$ of $\widetilde{J}_{n-1}(F)$, we put

$$
\mathrm{FJ}_{\psi_{\xi}}(\Pi)=\left(\Pi \otimes \overline{\omega_{\psi_{\xi}}}\right)_{V(F)} .
$$

Here, $(\cdot)_{V(F)}$ means the maximal quotient on which $V(F)$ acts trivially. We call $\mathrm{FJ}_{\psi_{\xi}}(\Pi)$ the Fourier-Jacobi module of $\Pi$ with index $\psi_{\xi}$. We regard $\mathrm{FJ}_{\psi_{\xi}}(\Pi)$ as a representation of $\widetilde{\operatorname{Sp}}_{n-1}(F)$. Since $V(F)$ is unipotent, the correspondence $\Pi \mapsto \mathrm{FJ}_{\psi_{\xi}}(\Pi)$ is an exact functor from the category of smooth representations of $\widetilde{J}_{n-1}(F)$ to the category of smooth representations of $\widetilde{\operatorname{Sp}}_{n-1}(F)$.

Conversely, for a smooth representation $\pi$ of $\widetilde{\operatorname{Sp}}_{n-1}(F)$, one can consider the tensor product

$$
\pi \otimes \omega_{\psi_{\xi}}
$$

which is a smooth representation of $\widetilde{J}_{n-1}(F)$. The correspondence $\pi \mapsto \pi \otimes \omega_{\psi_{\xi}}$ is an exact functor from the category of smooth representations of $\widetilde{\mathrm{Sp}}_{n-1}(F)$ to the category of smooth representations of $\widetilde{J}_{n-1}(F)$.

The following proposition seems to be well-known, but we give a proof for the convenience of the readers. (For an archimedean analogue, see [49].)

Proposition 2.1. Suppose that $F$ is non-archimedean. The map

$$
\pi \mapsto \pi \otimes \omega_{\psi_{\xi}}
$$

gives a 1-1 correspondence between irreducible smooth representations of $\widetilde{\operatorname{Sp}}_{n-1}(F)$ and irreducible smooth representations of $\widetilde{J}_{n-1}(F)$ on which $Z(F)$ acts by $\psi_{\xi}$. The inverse mapping is given by the Fourier-Jacobi module $\Pi \mapsto \mathrm{FJ}_{\psi_{\xi}}(\Pi)$.

Proof. Since any smooth representation of $V(F)$ on which $Z(F)$ acts by $\psi_{\xi}$ is a direct sum of copies of the Weil representation $\omega_{\psi_{\xi}}$, for any (nonzero) smooth representation $\Pi$ of $\widetilde{J}_{n-1}$, we can write $\Pi \cong \mathcal{V} \otimes \mathcal{S}(X(F))$ as a representation of $V(F)$ for some (nonzero) vector space $\mathcal{V}$ on which $V(F)$ acts trivially. For $g \in \widetilde{\mathrm{Sp}}_{n-1}(F)$, the operator $\Pi(g) \circ\left(\mathbf{1}_{\mathcal{V}} \otimes \omega_{\psi_{\xi}}(g)^{-1}\right)$ commutes with the action of $V(F)$. By Schur's lemma, we have

$$
\Pi(g) \circ\left(\mathbf{1}_{\mathcal{V}} \otimes \omega_{\psi_{\xi}}(g)^{-1}\right)=\pi(g) \otimes \mathbf{1}_{\mathcal{S}(X(F))}
$$

for some $\pi(g) \in \operatorname{Aut}(\mathcal{V})$. Then $\pi$ gives a group homomorphism $\pi: \widetilde{\operatorname{Sp}}_{n-1}(F) \rightarrow \operatorname{Aut}(\mathcal{V})$. It is smooth since $\Pi$ and $\omega_{\psi_{\xi}}$ are smooth. We conclude that $\Pi \cong \pi \otimes \omega_{\psi_{\xi}}$ as representations of $\widetilde{J}_{n-1}(F)$. Note that $\mathrm{FJ}_{\psi_{\xi}}\left(\pi \otimes \omega_{\psi_{\xi}}\right) \cong \pi$ as representations of $\widetilde{\operatorname{Sp}}_{n-1}(F)$. In particular, $\mathrm{FJ}_{\psi_{\xi}}(\Pi) \neq 0$ for any nonzero smooth representation of $\widetilde{J}_{n-1}(F)$. Since $-\otimes \omega_{\psi_{\xi}}$ and $\mathrm{FJ}_{\psi_{\xi}}$ are exact functors, we see that $\pi$ is irreducible as a representation of $\widetilde{\operatorname{Sp}}_{n-1}(F)$ if and only if $\pi \otimes \omega_{\psi_{\xi}}$ is irreducible as a representation of $\widetilde{J}_{n-1}(F)$. 
For an irreducible smooth representation $\Pi$ of $\widetilde{\operatorname{Sp}}_{n}(F)$, we denote by $\Pi_{\psi_{\xi}}$ the maximal quotient of $\Pi$ on which $Z(F)$ acts by $\psi_{\xi}$. Then $\Pi_{\psi_{\xi}}$ is a smooth representation of $\widetilde{J}_{n-1}(F)$. By a similar argument to the proof of Proposition 2.1, we have

$$
\Pi_{\psi_{\xi}} \cong \mathrm{FJ}_{\psi_{\xi}}\left(\Pi \mid \widetilde{J}_{n-1}(F)\right) \otimes \omega_{\psi_{\xi}} .
$$

Let $\mu$ be a unitary character of $F^{\times}$. For each integer $n$, we define a character $\mu^{(n)}$ of $\widetilde{M}_{n}(F)$ by

$$
\mu^{(n)}((\mathbf{m}(A), \zeta))=\zeta^{n}\left(\frac{\alpha_{\psi}(1)}{\alpha_{\psi}(\operatorname{det} A)}\right)^{n} \mu(\operatorname{det} A) .
$$

It is also regarded as a character of $\widetilde{P}_{n}(F)$. If

$$
\tau=\mu \times \mu^{-1}=\operatorname{Ind}_{P_{(1,1)}(F)}^{\mathrm{GL}_{2}(F)}\left(\mu \otimes \mu^{-1}\right)
$$

is an irreducible parabolic induction of $\mathrm{GL}_{2}(F)$, where $P_{(1,1)}$ is the Borel subgroup of $\mathrm{GL}_{2}$ consisting of upper triangular matrices, then the degenerate principal series

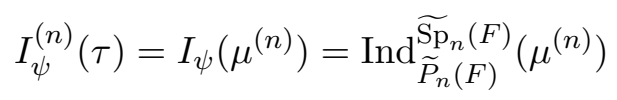

is irreducible by $[35,50]$. See also $[29$, Propositions $3.1,5.1]$.

In general, the Fourier-Jacobi module $\mathrm{FJ}_{\psi_{\xi}}\left(\Pi \mid \widetilde{J}_{n-1}(F)\right)$ is rarely irreducible since the restriction of $\Pi$ to $\widetilde{J}_{n-1}(F)$ is often reducible. However, $\mathrm{FJ}_{\psi_{\xi}}$ sends degenerate principal series of $\widetilde{\mathrm{Sp}_{n}}(F)$ to ones of $\widetilde{\operatorname{Sp}}_{n-1}(F)$.

Proposition 2.2. For any $\xi \in F^{\times}$, we have an isomorphism

$$
\mathrm{FJ}_{\psi_{\xi}}\left(I_{\psi}^{(n)}(\tau)\right) \cong I_{\psi}^{(n-1)}\left(\tau \chi_{\xi}\right)
$$

where $\tau \chi_{\xi}=\tau \otimes \chi_{\xi}$. In particular, we have

$$
\left(I_{\psi}^{(n)}(\tau)\right)_{\psi_{\xi}} \cong I_{\psi}^{(n-1)}\left(\tau \chi_{\xi}\right) \otimes \omega_{\psi_{\xi}}
$$

as $\widetilde{J}_{n-1}(F)$-modules.

Proof. See [21, Theorem 3.1] and [55].

2.4. Archimedean case. In this subsection, we consider the case where $F=\mathbb{R}$. We assume that the non-trivial unitary character $\psi$ of $\mathbb{R}$ is of the form

$$
\psi(x)=\exp (2 \pi a \sqrt{-1} x)
$$

for $x \in \mathbb{R}$ with $a>0$. Then the Weil constant $\alpha_{\psi}(t)$ is given by

$$
\alpha_{\psi}(t)= \begin{cases}\exp (\pi \sqrt{-1} / 4) & \text { if } t>0 \\ \exp (-\pi \sqrt{-1} / 4) & \text { if } t<0\end{cases}
$$

For $\xi \in \mathbb{R}^{\times}$with $\xi>0$, we define $\phi_{\xi}^{0} \in \mathcal{S}(X(\mathbb{R}))$ by

$$
\phi_{\xi}^{0}(x)=\exp \left(-2 \pi a \xi\left(x_{1}^{2}+\cdots+x_{n-1}^{2}\right)\right)
$$

for $x=\left(x_{1}, \ldots, x_{n-1}\right) \in X(\mathbb{R})$. Let

$$
K_{\infty}=\left\{\left(\begin{array}{cc}
\alpha & \beta \\
-\beta & \alpha
\end{array}\right) \mid \alpha, \beta \in \operatorname{Mat}_{n}(\mathbb{R}),{ }^{t} \alpha \beta={ }^{t} \beta \alpha,{ }^{t} \alpha \alpha+{ }^{t} \beta \beta=\mathbf{1}_{n}\right\}
$$


be the usual maximal compact subgroup of $\operatorname{Sp}_{n}(\mathbb{R})$. For $u=\left(\begin{array}{cc}\alpha & \beta \\ -\beta & \alpha\end{array}\right) \in K_{\infty}$, we write $\operatorname{det}(\alpha+$ $\sqrt{-1} \beta)=e^{\sqrt{-1} \theta}$ with $-\pi<\theta \leq \pi$, and we set

$$
\operatorname{det}^{1 / 2}(u, \zeta)=\zeta e^{\sqrt{-1} \theta / 2} .
$$

Then $\omega_{\psi_{\xi}}(u, \zeta) \phi_{\xi}^{0}=\operatorname{det}^{1 / 2}(u, \zeta) \cdot \phi_{\xi}^{0}$ for any $(u, \zeta) \in \widetilde{K}_{\infty} \cap \widetilde{\operatorname{Sp}}_{n-1}(\mathbb{R})$. In particular, $\operatorname{det}^{1 / 2}$ is a genuine character of $\widetilde{K}_{\infty}$. For an integer $l$, we set $\operatorname{det}^{l / 2}(u, \zeta)=\left(\operatorname{det}^{1 / 2}(u, \zeta)\right)^{l}$. We denote the irreducible lowest weight representation of $\widetilde{\mathrm{Sp}}_{n}(\mathbb{R})$ with lowest $\widetilde{K}_{\infty}$-type $\operatorname{det}^{l / 2}$ by $\mathcal{D}_{l / 2}^{(n)}$.

Let

$$
\mathfrak{H}_{n}=\left\{Z \in \operatorname{Mat}_{n}(\mathbb{C}) \mid{ }^{t} Z=Z, \operatorname{Im}(Z)>0\right\}
$$

be the Siegel upper half space of genus $n$. Here, for a symmetric matrix $B$, we write $B>0$ if $B$ is positive definite. Then $\operatorname{Sp}_{n}(\mathbb{R})$ acts on $\mathfrak{H}_{n}$ by

$$
g(Z)=(A Z+B)(C Z+D)^{-1}, \quad g=\left(\begin{array}{cc}
A & B \\
C & D
\end{array}\right) \in \operatorname{Sp}_{n}(\mathbb{R}) .
$$

Note that the stabilizer of $\mathbf{i}=\sqrt{-1} \cdot \mathbf{1}_{n} \in \mathfrak{H}_{n}$ in $\operatorname{Sp}_{n}(\mathbb{R})$ is equal to $K_{\infty}$. We set $j(g, Z)=$ $\operatorname{det}(C Z+D)$. Then there exists a unique automorphy factor $\widetilde{j}((g, \zeta), Z)$ of $\widetilde{\operatorname{Sp}}_{n}(\mathbb{R})$ such that $\widetilde{j}((g, \zeta), Z)^{2}=j(g, Z)$ for any $(g, \zeta) \in \widetilde{\mathrm{Sp}}_{n}(\mathbb{R})$ and $Z \in \mathfrak{H}_{n}$. The following lemma might be wellknown, but we give a proof for the convenience of the readers.

Lemma 2.3. For $A \in \mathrm{GL}_{n}(\mathbb{R})$ with $\operatorname{det} A>0, B \in \operatorname{Sym}_{n}(\mathbb{R})$, and $(u, \zeta) \in \widetilde{K}_{\infty}$, we have

$$
\widetilde{j}(\mathbf{n}(B)(\mathbf{m}(A), 1)(u, \zeta), \mathbf{i})=(\operatorname{det} A)^{-\frac{1}{2}} \operatorname{det}^{-1 / 2}(u, \zeta) .
$$

Proof. Since $\widetilde{j}((g, \zeta), Z)$ is an automorphy factor of $\widetilde{\mathrm{Sp}_{n}}(\mathbb{R})$, for any $(g, \zeta) \in \widetilde{\operatorname{Sp}}_{n}(\mathbb{R})$, the function

$$
\mathfrak{H}_{n} \rightarrow \mathbb{C}, Z \mapsto \tilde{j}((g, \zeta), Z)
$$

is holomorphic.

First, we claim that $\widetilde{j}(\mathbf{n}(B), Z)=1$ for any $B \in \operatorname{Sym}_{n}(\mathbb{R})$ and $Z \in \mathfrak{H}_{n}$. Note that $\widetilde{j}(\mathbf{n}(B), Z)^{2}=$ $j(\mathbf{n}(B), Z)=1$. Since $\mathfrak{H}_{n}$ is connected, $\widetilde{j}(\mathbf{n}(B), Z)$ is independent of $Z$. In particular, $B \mapsto$ $\widetilde{j}(\mathbf{n}(B), Z)$ gives a group homomorphism $\operatorname{Sym}_{n}(\mathbb{R}) \rightarrow\{ \pm 1\}$. Since $\operatorname{Sym}_{n}(\mathbb{R})$ is divisible, it must be the trivial character. Hence $\widetilde{j}(\mathbf{n}(B), Z)=1$.

Next, we claim that $\widetilde{j}((\mathbf{m}(A), 1), Z)=(\operatorname{det} A)^{-1 / 2}$ for $A \in \mathrm{GL}_{2}(\mathbb{R})$ with $\operatorname{det} A>0$ and $Z \in \mathfrak{H}_{n}$. Since $\mathfrak{H}_{n}$ is connected, the function $\mathfrak{H}_{n} \ni Z \mapsto(\operatorname{det} A)^{1 / 2} \widetilde{j}((\mathbf{m}(A), 1), Z) \in\{ \pm 1\}$ is a constant for any $A \in \mathrm{GL}_{2}(\mathbb{R})$ with $\operatorname{det} A>0$. In particular, the map $A \mapsto(\operatorname{det} A)^{1 / 2} \widetilde{j}((\mathbf{m}(A), 1), Z) \in\{ \pm 1\}$ gives a group homomorphism. Since $\left(e^{(1 / 2) X}\right)^{2}=e^{X}$ for $X \in \mathfrak{g l}_{n}(\mathbb{R})$, we have $(\operatorname{det} A)^{1 / 2} \widetilde{j}((\mathbf{m}(A), 1), Z)=1$ when $A=e^{X}$ for some $X \in \mathfrak{g l}_{n}(\mathbb{R})$. Since any $A \in \mathrm{GL}_{n}(\mathbb{R})$ with $\operatorname{det} A>0$ can be written as a product $A=e^{X_{1}} \cdots e^{X_{k}}$ for some $X_{1}, \ldots, X_{k} \in \mathfrak{g l}_{n}(\mathbb{R})$, we have $(\operatorname{det} A)^{1 / 2} \widetilde{j}((\mathbf{m}(A), 1), Z)=1$ for any $A \in \mathrm{GL}_{2}(\mathbb{R})$ with $\operatorname{det} A>0$.

Similarly, $\widetilde{j}\left(\left(\mathbf{1}_{2 n},-1\right), Z\right) \in\{ \pm 1\}$ is independent of $Z \in \mathfrak{H}_{n}$. Choose $A \in \mathrm{O}(n)$ such that $A^{2}=\mathbf{1}_{n}$ and $\operatorname{det} A=-1$. Then $(\mathbf{m}(A), 1)^{2}=\left(\mathbf{1}_{2 n},-1\right)$ so that $\widetilde{j}\left(\left(\mathbf{1}_{2 n},-1\right), \mathbf{i}\right)=\widetilde{j}((\mathbf{m}(A), 1), \mathbf{i})^{2}=$ $j(\mathbf{m}(A), \mathbf{i})=\operatorname{det}(A)^{-1}=-1$. Hence $\widetilde{j}\left(\left(\mathbf{1}_{2 n},-1\right), Z\right)=-1$ for any $Z \in \mathfrak{H}_{n}$.

Finally, we see that $\operatorname{det}^{1 / 2}(u, \zeta) \widetilde{j}((u, \zeta), \mathbf{i}) \in\{ \pm 1\}$ gives a group homomorphism $\widetilde{K}_{\infty} \rightarrow\{ \pm 1\}$, which factors through $K_{\infty}$. Since $K_{\infty}$ is divisible, it must be the trivial character. Hence $\widetilde{j}((u, \zeta), \mathbf{i})=$ $\operatorname{det}^{-1 / 2}(u, \zeta)$. This completes the proof. 
Let $\operatorname{Sym}_{n}^{+}(\mathbb{R})=\left\{B \in \operatorname{Sym}_{n}(\mathbb{R}) \mid B>0\right\}$. Fix an integer $k>0$. For $B \in \operatorname{Sym}_{n}^{+}(\mathbb{R})$, we define a function $W_{B}^{0}$ on $\widetilde{\mathrm{Sp}_{n}}(\mathbb{R})$ by

$$
\begin{aligned}
W_{B}^{0}(g) & =(\operatorname{det} B)^{(2 k+n) / 4} \psi(\operatorname{Tr}(B g(\mathbf{i}))) \widetilde{j}(g, \mathbf{i})^{-(2 k+n)} \\
& =\psi(\operatorname{Tr}(B z)) \operatorname{det}(B[A])^{(2 k+n) / 4} \exp (-2 \pi a \cdot \operatorname{Tr}(B[A])) \operatorname{det}^{k+(n / 2)}(\widetilde{u})
\end{aligned}
$$

for $g=\mathbf{n}(z)(\mathbf{m}(A), 1) \widetilde{u} \in \widetilde{\operatorname{Sp}}_{n}(\mathbb{R})$ with $z \in \operatorname{Sym}_{n}(\mathbb{R}), A \in \mathrm{GL}_{n}(\mathbb{R})$, $\operatorname{det} A>0$, and $\widetilde{u} \in \widetilde{K}_{\infty}$. Here, we put $B[A]={ }^{t} A B A$.

Proposition 2.4 ([29, Lemma 7.6]). For $\xi \in \mathbb{R}^{\times}$with $\xi>0$ and $B^{\prime} \in \operatorname{Sym}_{n-1}^{+}(\mathbb{R})$, we put

$$
B=\left(\begin{array}{cc}
\xi & 0 \\
0 & B^{\prime}
\end{array}\right) \in \operatorname{Sym}_{n}^{+}(\mathbb{R})
$$

Then we have

$$
\int_{X(\mathbb{R})} W_{B}^{0}\left(\mathbf{v}(x, 0,0) g^{\prime}\right) \overline{\omega_{\psi_{\xi}}\left(g^{\prime}\right) \phi_{\xi}^{0}(x)} d x=|\operatorname{det} B|^{1 / 4} e^{-2 \pi a \xi} W_{B^{\prime}}^{0}\left(g^{\prime}\right)
$$

for $g^{\prime} \in{\widetilde{\mathrm{Sp}_{n-1}}}(\mathbb{R})$.

The Lie algebras $\mathfrak{s p}_{n}(\mathbb{R})$ and $\mathfrak{k}$ of $\operatorname{Sp}_{n}(\mathbb{R})$ and $K_{\infty}$ are given by

$$
\begin{aligned}
\mathfrak{s p}_{n}(\mathbb{R}) & =\left\{X \in \operatorname{Mat}_{2 n}(\mathbb{R}) \mid{ }^{t} X\left(\begin{array}{cc}
0 & -\mathbf{1}_{n} \\
\mathbf{1}_{n} & 0
\end{array}\right)+{ }^{t} X\left(\begin{array}{cc}
0 & -\mathbf{1}_{n} \\
\mathbf{1}_{n} & 0
\end{array}\right) X=0\right\} \\
& =\left\{\left(\begin{array}{cc}
A & B \\
C & D
\end{array}\right) \in \operatorname{Mat}_{2 n}(\mathbb{R}) \mid B={ }^{t} B, C={ }^{t} C, A=-{ }^{t} D\right\}, \\
\mathfrak{k} & =\left\{\left(\begin{array}{cc}
A & B \\
-B & A
\end{array}\right) \in \operatorname{Mat}_{2 n}(\mathbb{R}) \mid{ }^{t} A=-A,{ }^{t} B=B\right\},
\end{aligned}
$$

respectively. Note that $\mathfrak{k}$ is the 1-eigenspace of the Cartan involution $\theta X=-{ }^{t} X$ on $\mathfrak{s p}_{n}(\mathbb{R})$. The $(-1)$-eigenspace is given by

$$
\mathfrak{p}=\left\{\left(\begin{array}{cc}
A & B \\
B & -A
\end{array}\right) \in \operatorname{Mat}_{2 n}(\mathbb{R}) \mid A={ }^{t} A, B={ }^{t} B\right\} .
$$

Hence $\mathfrak{s p}_{n}(\mathbb{R})=\mathfrak{k} \oplus \mathfrak{p}$. The homeomorphism

$$
\rho: \operatorname{Sp}_{n}(\mathbb{R}) / K_{\infty} \rightarrow \mathfrak{H}_{n}, g \mapsto g(\mathbf{i})
$$

induces an isomorphism

$$
d \rho: \mathfrak{s p}_{n}(\mathbb{R}) / \mathfrak{k}=\mathfrak{p} \stackrel{\sim}{\rightarrow} T_{\mathfrak{i}} \mathfrak{H}_{n} \cong \operatorname{Sym}_{n}(\mathbb{C}),
$$

where $T_{\mathbf{i}} \mathfrak{H}_{n}$ is the tangent space at $\mathbf{i}$ on $\mathfrak{H}_{n}$. This map is given by

$$
d \rho\left(\left(\begin{array}{cc}
A & B \\
B & -A
\end{array}\right)\right)=2(B+\sqrt{-1} A) .
$$

Then the complex structure (i.e., multiplication with $\sqrt{-1}$ ) on $\operatorname{Sym}_{n}(\mathbb{C})$ gives a map

$$
J: \mathfrak{p} \rightarrow \mathfrak{p},\left(\begin{array}{cc}
A & B \\
B & -A
\end{array}\right) \mapsto\left(\begin{array}{cc}
B & -A \\
-A & -B
\end{array}\right) .
$$

Let $\mathfrak{s p}_{n}(\mathbb{C}), \mathfrak{k}_{\mathbb{C}}$, and $\mathfrak{p}_{\mathbb{C}}$ be the complexifications of $\mathfrak{s p}_{n}(\mathbb{R}), \mathfrak{k}$, and $\mathfrak{p}$, respectively. We denote the $( \pm \sqrt{-1})$-eigenspace of $J$ on $\mathfrak{p}_{\mathbb{C}}$ by $\mathfrak{p}_{\mathbb{C}}^{ \pm}$. Then

$$
\mathfrak{p}_{\mathbb{C}}^{ \pm}=\left\{\left(\begin{array}{cc}
A & B \\
B & -A
\end{array}\right) \otimes 1 \pm\left(\begin{array}{cc}
-B & A \\
A & B
\end{array}\right) \otimes \sqrt{-1} \mid A, B \in \operatorname{Sym}_{n}(\mathbb{R})\right\}
$$




$$
=\left\{\left(\begin{array}{cc}
A & \pm \sqrt{-1} A \\
\pm \sqrt{-1} A & -A
\end{array}\right) \in \operatorname{Mat}_{2 n}(\mathbb{C}) \mid A \in \operatorname{Sym}_{n}(\mathbb{C})\right\} .
$$

The elements of $\mathfrak{p}_{\mathbb{C}}^{+}$and $\mathfrak{p}_{\mathbb{C}}^{-}$correspond to the linear combinations of differential operators

$$
\left\{\frac{d}{d z_{j}}\right\}_{j} \quad \text { and } \quad\left\{\frac{d}{d \overline{z_{j}}}\right\}_{j},
$$

respectively, where $z_{j}=x_{j}+\sqrt{-1} y_{j}$ are coordinates on $\mathfrak{H}_{n}$ at the point $\mathbf{i}$.

The element $X \in \mathfrak{p}$ acts on a smooth function $W$ on $\widetilde{\mathrm{Sp}_{n}}(\mathbb{R})$ by

$$
X \cdot W(g)=\left.\frac{d}{d t}\right|_{t=0} W\left(g\left(e^{t X}, 1\right)\right)
$$

for $g \in \widetilde{\mathrm{Sp}}_{n}(\mathbb{R})$. This action is extended to $\mathfrak{p}_{\mathbb{C}}$ linearly. It is easy to check that $\mathfrak{p}_{\mathbb{C}}^{-}$acts on $W_{B}^{0}$ by zero for any $B \in \operatorname{Sym}_{n}^{+}(\mathbb{R})$ (cf. [4, Lemma 7]). Hence $W_{B}^{0}$ is a lowest weight vector in $\mathcal{D}_{k+(n / 2)}^{(n)}$. Similarly, $\mathfrak{p}_{\mathbb{C}}^{-} \cap \mathfrak{s p}_{n-1}(\mathbb{C})$ acts on $\phi_{\xi}^{0}$ by zero. This fact can be proven by using the Fock model of $\omega_{\psi_{\xi}}$.

2.5. Global case. Now we let $F$ be a totally real number field, and $\psi$ be a non-trivial unitary character of $\mathbb{A} / F$. We assume that for each infinite place $v$ of $F$, there exists $a_{v} \in F_{v} \cong \mathbb{R}$ with $a_{v}>0$ such that $\psi\left(x_{v}\right)=\exp \left(2 \pi a_{v} \sqrt{-1} x_{v}\right)$ for $x_{v} \in F_{v}$. For a place $v$ of $F$, we define a maximal compact subgroup $K_{v}$ of $\operatorname{Sp}_{n}\left(F_{v}\right)$ by

$$
K_{v}= \begin{cases}\operatorname{Sp}_{n}\left(\mathfrak{o}_{v}\right) & \text { if } v \text { is non-archimedean, } \\ K_{\infty} & \text { if } v \text { is real. }\end{cases}
$$

Here, we denote by $\mathfrak{o}_{v}$ the ring of integers of $F_{v}$ when $v$ is non-archimedean. If $v$ is archimedean, we define $\left(\mathfrak{p}_{v}\right)_{\mathbb{C}}^{-} \subset \operatorname{Lie}\left(\operatorname{Sp}_{n}\left(F_{v}\right)\right) \otimes_{\mathbb{R}} \mathbb{C}$ as in the previous subsection.

Recall that a function $\varphi: \operatorname{Sp}_{n}(F) \backslash \widetilde{\operatorname{Sp}_{n}}(\mathbb{A}) \rightarrow \mathbb{C}$ is a cusp form if

- $\varphi$ is smooth and of moderate growth;

- $\varphi$ is right $\widetilde{K}$-finite, where $\widetilde{K}=\prod_{v} \widetilde{K}_{v}$;

- $\varphi$ is $\mathfrak{z}$-finite, where $\mathfrak{z}$ is the center of the universal enveloping algebra of $\operatorname{Lie}\left(\operatorname{Sp}_{n}\left(F \otimes_{\mathbb{Q}} \mathbb{R}\right)\right) \otimes_{\mathbb{R}} \mathbb{C}$;

- there exists $\delta \in\{0,1\}$ such that $\varphi((g, \zeta))=\zeta^{\delta} \varphi((g, 1))$ for any $g \in \operatorname{Sp}_{n}(\mathbb{A})$;

- For any proper $F$-parabolic subgroup $P$ of $\mathrm{Sp}_{n}$, the constant term along $P$

$$
\int_{N(F) \backslash N(\mathbb{A})} \varphi(u g) d u
$$

is zero for any $g \in \widetilde{\operatorname{Sp}}_{n}(\mathbb{A})$, where $N$ is the unipotent radical of $P$.

We say that $\varphi$ is genuine if $\delta=1$. Let $l=\left(l_{v}\right)_{v} \in \prod_{v \mid \infty} \mathbb{Z}$. We say that a cusp form $\varphi$ is holomorphic of weight $l / 2$ if

- $X_{v} \cdot \varphi=0$ for any $X_{v} \in\left(\mathfrak{p}_{v}\right)_{\mathbb{C}}^{-}$

- $\varphi\left(g \widetilde{u}_{v}\right)=\operatorname{det}^{l_{v} / 2}\left(\widetilde{u}_{v}\right) \varphi(g)$ for $g \in \widetilde{\operatorname{Sp}}_{n}(\mathbb{A})$ and $\widetilde{u}_{v} \in \widetilde{K}_{v}$

for any infinite place $v$. We denote the space of holomorphic cusp forms of weight $l / 2$ by

$$
\mathcal{S}_{l / 2}\left(\operatorname{Sp}_{n}(F) \backslash \widetilde{\operatorname{Sp}}_{n}(\mathbb{A})\right)
$$

The group $\widetilde{\operatorname{Sp}}_{n}\left(\mathbb{A}_{\text {fin }}\right)$ acts on $\mathcal{S}_{l / 2}\left(\operatorname{Sp}_{n}(F) \backslash \widetilde{\operatorname{Sp}}_{n}(\mathbb{A})\right)$ by the right translation.

Let $\tau=\otimes_{v}^{\prime} \tau_{v}$ be an irreducible unitary cuspidal automorphic representation of $\mathrm{GL}_{2}(\mathbb{A})$. Assume that 
(A1) for any finite place $v$, the local factor $\tau_{v}$ is an irreducible principal representation $\mu_{v} \times \mu_{v}^{-1}$;

(A2) for any infinite place $v$, the local factor $\tau_{v}$ is a discrete series representation with lowest weight $\pm 2 k_{v}$ where $k_{v}>0$;

(A3) the root number

$$
\varepsilon(\tau)=\varepsilon\left(\frac{1}{2}, \tau, \psi\right)=\prod_{v<\infty} \mu_{v}(-1) \cdot \prod_{v \mid \infty}(-1)^{k_{v}}
$$

is equal to 1 .

For $v<\infty$, we notice that $\left|\mu_{v}\left(\varpi_{v}\right)\right|_{v}=1$ by the Ramanujan conjecture proven in the case of Hilbert modular forms by Blasius [8], where $\varpi_{v}$ is a uniformizer of $F_{v}$. For a more general result on the Ramanujan conjecture, see [10]. We refer to $\pm 2 k=\left( \pm 2 k_{v}\right)_{v} \in \prod_{v \mid \infty} \mathbb{Z}$ as the weight of $\otimes_{v \mid \infty} \tau_{v}$.

Put $k+(n / 2)=\left(k_{v}+n / 2\right)_{v} \in \prod_{v \mid \infty} \mathbb{Z}$ for each integer $n>0$. For $v<\infty$, we let $I_{\psi_{v}}^{(n)}\left(\tau_{v}\right)=$ $\operatorname{Ind}_{\widetilde{P}_{n}\left(F_{v}\right)} \widetilde{\mathrm{Sp}}_{n}\left(F_{v}\right)\left(\mu_{v}^{(n)}\right)$ be the degenerate principal series defined in $\S 2.3$. Set $I_{\psi}^{(n)}(\tau)=\otimes_{v<\infty}^{\prime} I_{\psi_{v}}^{(n)}\left(\tau_{v}\right)$, which is an irreducible representation of $\widetilde{\mathrm{Sp}_{n}}\left(\mathbb{A}_{\mathrm{fin}}\right)$. The following is a part of the main theorem in [29].

Theorem 2.5 ([29, Theorems 1.1, 1.2]). Let $\tau=\otimes_{v}^{\prime} \tau_{v}$ be an irreducible unitary cuspidal automorphic representation of $\mathrm{GL}_{2}(\mathbb{A})$ satisfying the conditions (A1), (A2) and (A3). Then the representation $I_{\psi}^{(n)}(\tau)$ occurs in $\mathcal{S}_{k+(n / 2)}\left(\operatorname{Sp}_{n}(F) \backslash \widetilde{\operatorname{Sp}}_{n}(\mathbb{A})\right)$ with multiplicity one.

We denote the unique subrepresentation of $\mathcal{S}_{k+(n / 2)}\left(\operatorname{Sp}_{n}(F) \backslash \widetilde{\operatorname{Sp}_{n}}(\mathbb{A})\right)$ which is isomorphic to $I_{\psi}^{(n)}(\tau)$ by $\operatorname{Ik}_{\psi}^{(n)}(\tau)$, and call it the Ikeda lift of $\tau$.

Let $\xi \in F^{\times}$be a totally positive element. For $\phi \in \mathcal{S}(X(\mathbb{A}))$, the theta function $\Theta_{\psi_{\xi}}^{\phi}\left(v g^{\prime}\right)$ is defined by

$$
\Theta_{\psi_{\xi}}^{\phi}\left(\mathbf{v}(x, y, z) g^{\prime}\right)=\sum_{t \in X(F)} \psi_{\xi}\left(z+2 t \cdot{ }^{t} y+x \cdot{ }^{t} y\right) \omega_{\psi_{\xi}}\left(g^{\prime}\right) \phi(t+x)
$$

for $\mathbf{v}(x, y, z) \in V(\mathbb{A})$ and $g^{\prime} \in \widetilde{\mathrm{Sp}}_{n-1}(\mathbb{A})$. It is a genuine automorphic form on $\widetilde{J}_{n-1}(\mathbb{A})$. One can easily check that

$$
\int_{Z(\mathbb{A}) V(F) \backslash V(\mathbb{A})} \Theta_{\psi_{\xi}}^{\phi_{1}}\left(v g^{\prime}\right) \overline{\Theta_{\psi_{\xi}}^{\phi_{2}}\left(v g^{\prime}\right)} d v=\left(\phi_{1}, \phi_{2}\right)=\int_{X(\mathbb{A})} \phi_{1}(x) \overline{\phi_{2}(x)} d x
$$

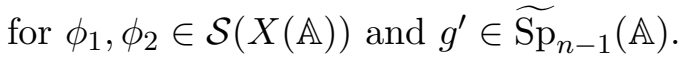

We denote by $\mathcal{S}(X(\mathbb{A}))_{\xi}$ the subspace of $\mathcal{S}(X(\mathbb{A}))$ spanned by $\phi=\otimes_{v} \phi_{v}$ such that $\phi_{v}=\phi_{\xi_{v}}^{0}$ for each infinite place $v$. For $\varphi \in \mathcal{S}_{l / 2}\left(\operatorname{Sp}_{n}(F) \backslash \widetilde{\mathrm{Sp}_{n}}(\mathbb{A})\right)$ and $\phi \in \mathcal{S}(X(\mathbb{A}))_{\xi}$, the Fourier-Jacobi coefficient associated to $(\varphi, \phi)$ is defined by

$$
\mathrm{FJ}_{\psi_{\xi}}^{\phi}\left(g^{\prime} ; \varphi\right)=\int_{V(F) \backslash V(\mathbb{A})} \varphi\left(v g^{\prime}\right) \overline{\Theta_{\psi_{\xi}}^{\phi}\left(v g^{\prime}\right)} d v .
$$

This is a cusp form on $\widetilde{\mathrm{Sp}}_{n-1}(\mathbb{A})$. (See also [19, Lemma 2.3] and [18, Theorem 8].) Moreover, by Proposition 2.4, we conclude that

$$
\mathrm{FJ}_{\psi_{\xi}}^{\phi}(\varphi) \in \mathcal{S}_{(l-1) / 2}\left(\operatorname{Sp}_{n-1}(F) \backslash \widetilde{\operatorname{Sp}}_{n-1}(\mathbb{A})\right)
$$

for any $\varphi \in \mathcal{S}_{l / 2}\left(\operatorname{Sp}_{n}(F) \backslash \widetilde{\operatorname{Sp}}_{n}(\mathbb{A})\right)$ and $\phi \in \mathcal{S}(X(\mathbb{A}))_{\xi}$. 
Proposition 2.6. For $\mathcal{F} \in \mathrm{Ik}_{\psi}^{(n)}(\tau)$ and $\phi \in \mathcal{S}(X(\mathbb{A}))_{\xi}$, we have $\mathrm{FJ}_{\psi_{\xi}}^{\phi}(\mathcal{F}) \in \mathrm{Ik}_{\psi}^{(n-1)}\left(\tau \chi_{\xi}\right)$.

Proof. We may assume that $\mathrm{FJ}_{\psi_{\xi}}^{\phi}(\mathcal{F}) \neq 0$. Let $\Pi$ be the representation of $\widetilde{\operatorname{Sp}}_{n-1}(\mathbb{A})$ generated by $\mathrm{FJ}_{\psi_{\xi}}^{\phi}(\mathcal{F})$ for $\mathcal{F} \in \mathrm{Ik}_{\psi}^{(n)}(\tau)$ and $\phi \in \mathcal{S}(X(\mathbb{A}))_{\xi}$. Since $\Pi$ is cuspidal, it is a direct sum of irreducible representations. Choose an irreducible direct summand $\pi$ of $\Pi$ and a projection $\Pi \rightarrow \pi$. Then the map

$$
\mathrm{Ik}_{\psi}^{(n)}(\tau) \otimes \overline{\omega_{\psi_{\xi}}} \stackrel{\mathrm{FJ}_{\psi_{\xi}}}{\longrightarrow} \Pi \rightarrow \pi
$$

is a (nonzero) $V\left(\mathbb{A}_{\text {fin }}\right)$-invariant map so that it factors through $\mathrm{FJ}_{\psi_{\xi}}\left(\operatorname{Ik}_{\psi}^{(n)}(\tau)\right)$. Since $\mathrm{FJ}_{\psi_{\xi}}\left(\mathrm{Ik}_{\psi}^{(n)}(\tau)\right)$ is nonzero, we have $\mathrm{FJ}_{\psi_{\xi}}\left(\mathrm{Ik}_{\psi}^{(n)}(\tau)\right)=\mathrm{Ik}_{\psi}^{(n-1)}\left(\tau \chi_{\xi}\right)$ by Proposition 2.2. Since it is irreducible, we have $\pi \cong \operatorname{Ik}_{\psi}^{(n-1)}\left(\tau \chi_{\xi}\right)$. Hence $\Pi$ is isomorphic to a direct sum of some copies of $\operatorname{Ik}_{\psi}^{(n-1)}\left(\tau \chi_{\xi}\right)$. However the Ikeda lift appears in $\mathcal{S}_{k+(n-1) / 2}\left(\operatorname{Sp}_{n-1}(F) \backslash \widetilde{\operatorname{Sp}}_{n-1}(\mathbb{A})\right)$ with multiplicity one, we see that $\Pi$ is irreducible and is equal to $\operatorname{Ik}_{\psi}^{(n-1)}\left(\tau \chi_{\xi}\right)$.

For $\varphi \in \mathcal{S}_{l / 2}\left(\operatorname{Sp}_{n}(F) \backslash \widetilde{\operatorname{Sp}}_{n}(\mathbb{A})\right)$, we define the $\xi$-th Fourier-Jacobi coefficient $\varphi_{\psi_{\xi}}$ by

$$
\varphi_{\psi_{\xi}}\left(v g^{\prime}\right)=\int_{Z(F) \backslash Z(\mathbb{A})} \varphi\left(z v g^{\prime}\right) \overline{\psi_{\xi}(z)} d z
$$

for $v \in V(\mathbb{A})$ and $g^{\prime} \in{\widetilde{\operatorname{Sp}_{n-1}}}(\mathbb{A})$. If $\Pi$ be a subspace of $\mathcal{S}_{l / 2}\left(\operatorname{Sp}_{n}(F) \backslash \widetilde{\operatorname{Sp}_{n}}(\mathbb{A})\right)$, we put

$$
\Pi_{\psi_{\xi}}=\left\{\varphi_{\psi_{\xi}} \mid \varphi \in \Pi\right\} \text {. }
$$

This is the maximal quotient of $\Pi$ on which $Z\left(\mathbb{A}_{\text {fin }}\right)$ acts by $\psi_{\xi}$.

Proposition 2.7. $\quad(1)$ For $\mathcal{F} \in \mathrm{Ik}_{\psi}^{(n)}(\tau)$, there exist $\mathcal{F}_{1}^{\prime}, \ldots, \mathcal{F}_{r}^{\prime} \in \mathrm{Ik}_{\psi}^{(n-1)}\left(\tau \chi_{\xi}\right)$ and $\phi_{1}, \ldots, \phi_{r} \in$ $\mathcal{S}(X(\mathbb{A}))_{\xi}$ such that

$$
\mathcal{F}_{\psi_{\xi}}\left(v g^{\prime}\right)=\sum_{i=1}^{r} \mathcal{F}_{i}^{\prime}\left(g^{\prime}\right) \Theta_{\psi_{\xi}}^{\phi_{i}}\left(v g^{\prime}\right)
$$

for $v \in V(\mathbb{A})$ and $g^{\prime} \in \widetilde{\operatorname{Sp}}_{n-1}(\mathbb{A})$.

(2) Suppose that $\left(\operatorname{Ik}_{\psi}^{(n)}(\tau)\right)_{\psi_{\xi}}$ is nonzero. Then for $\mathcal{F}^{\prime} \in \mathrm{Ik}_{\psi}^{(n-1)}\left(\tau \chi_{\xi}\right)$ and $\phi \in \mathcal{S}(X(\mathbb{A}))_{\xi}$, there exists $\mathcal{F} \in \mathrm{Ik}_{\psi}^{(n)}(\tau)$ such that

$$
\mathcal{F}_{\psi_{\xi}}\left(v g^{\prime}\right)=\mathcal{F}^{\prime}\left(g^{\prime}\right) \Theta_{\psi_{\xi}}^{\phi}\left(v g^{\prime}\right)
$$

for $v \in V(\mathbb{A})$ and $g^{\prime} \in \widetilde{\operatorname{Sp}}_{n-1}(\mathbb{A})$.

(3) If $n \geq 2$, then $\left(\operatorname{Ik}_{\psi}^{(n)}(\tau)\right)_{\psi_{\xi}} \neq 0$ for any totally positive $\xi \in F^{\times}$.

Proof. By [26, Proposition 1.3], for $\mathcal{F} \in \mathrm{Ik}_{\psi}^{(n)}(\tau)$, there exist $\phi_{1}, \ldots, \phi_{r} \in \mathcal{S}(X(\mathbb{A}))_{\xi}$ with $\left(\phi_{i}, \phi_{j}\right)=$ $\delta_{i, j}$ such that

$$
\mathcal{F}_{\psi_{\xi}}\left(v g^{\prime}\right)=\sum_{i=1}^{r} \mathrm{FJ}_{\psi_{\xi}}^{\phi_{i}}(\mathcal{F})\left(g^{\prime}\right) \cdot \Theta_{\psi_{\xi}}^{\phi_{i}}\left(v g^{\prime}\right)
$$

for $v \in V(\mathbb{A})$ and $g^{\prime} \in{\widetilde{\mathrm{Sp}_{n-1}}}_{n}(\mathbb{A})$. Since $\mathrm{FJ}_{\psi_{\xi}}^{\phi_{i}}(\mathcal{F})\left(g^{\prime}\right) \in \mathrm{Ik}_{\psi}^{(n-1)}\left(\tau \chi_{\xi}\right)$ by Proposition 2.6, we obtain (1). 
Therefore, there is a $\widetilde{J}_{n-1}\left(\mathbb{A}_{\text {fin }}\right)$-equivariant inclusion

$$
\left(\mathrm{Ik}_{\psi}^{(n)}(\tau)\right)_{\psi_{\xi}} \subset \mathrm{Ik}_{\psi}^{(n-1)}\left(\tau \chi_{\xi}\right) \otimes \omega_{\psi_{\xi}}
$$

If $\left(\operatorname{Ik}_{\psi}^{(n)}(\tau)\right)_{\psi_{\xi}}$ is nonzero, this inclusion must be equal since $\operatorname{Ik}_{\psi}^{(n-1)}\left(\tau \chi_{\xi}\right) \otimes \omega_{\psi_{\xi}}$ is irreducible. Hence we obtain $(2)$.

Let $\operatorname{Sym}_{n}^{+}(F)$ be the subset of $\operatorname{Sym}_{n}(F)$ consisting of symmetric matrices whose images in $\operatorname{Sym}_{n}\left(F_{v}\right)$ are positive definite for each infinite place $v$. By [29, Lemmas 8.5, 5.4], there exists $\mathcal{F} \in \operatorname{Ik}_{\psi}^{(n)}(\tau)$ such that for $B \in \operatorname{Sym}_{n}^{+}(F)$, the $B$-th Fourier coefficient

$$
W_{B}(g)=\int_{\operatorname{Sym}_{n}(F) \backslash \operatorname{Sym}_{n}(\mathbb{A})} \mathcal{F}(\mathbf{n}(X) g) \overline{\psi(\operatorname{Tr}(B X))} d X
$$

is not identically zero if and only if $L\left(1 / 2, \tau \otimes \chi_{\operatorname{det}(B)}\right) \neq 0$. When $B=\operatorname{diag}\left(\xi, \xi^{\prime}, 1, \ldots, 1\right)$ with $\xi^{\prime} \in F^{\times}$totally positive, the $B$-th Fourier coefficient $W_{B}$ is given by

$$
W_{B}(g)=\int_{Z(\mathbb{A}) \operatorname{Sym}_{n}(F) \backslash \operatorname{Sym}_{n}(\mathbb{A})} \mathcal{F}_{\psi_{\xi}}(\mathbf{n}(X) g) \overline{\psi(\operatorname{Tr}(B X))} d X .
$$

Hence the $\xi$-th Fourier-Jacobi coefficient $\mathcal{F}_{\psi_{\xi}}$ is not identically zero if $W_{B} \neq 0$. Since there exists a totally positive element $\xi^{\prime} \in F^{\times}$such that $L\left(1 / 2, \tau \otimes \chi_{\xi \xi^{\prime}}\right) \neq 0$ by [53, Théorème 4$]$, we obtain $(3)$.

\section{Local MiYaWAKi Liftings}

In this section, we define the local Miyawaki lifting, and prove basic properties.

3.1. Definition. Let $F$ be a non-archimedean local field of characteristic zero, and $\psi$ be a nontrivial additive character of $F$. For two non-negative integers $n$ and $r$, we define an embedding $\iota: \mathrm{Sp}_{n} \times \mathrm{Sp}_{r} \hookrightarrow \mathrm{Sp}_{n+r}$ by

$$
\iota\left(\left(\begin{array}{ll}
A_{1} & B_{1} \\
C_{1} & D_{1}
\end{array}\right),\left(\begin{array}{ll}
A_{2} & B_{2} \\
C_{2} & D_{2}
\end{array}\right)\right)=\left(\begin{array}{cc|cc}
A_{1} & 0 & B_{1} & 0 \\
0 & A_{2} & 0 & B_{2} \\
\hline C_{1} & 0 & D_{1} & 0 \\
0 & C_{2} & 0 & D_{2}
\end{array}\right),
$$

and we identify $\mathrm{Sp}_{n} \times \mathrm{Sp}_{r}$ with the image. For $\tau=\mu \times \mu^{-1}$ with $\mu$ being a unitary character of $F^{\times}$, we consider the local Ikeda lift $I_{\psi}^{(n+r)}(\tau)=\operatorname{Ind}_{\widetilde{P}_{n+r}(F)}^{\widetilde{\mathrm{Sp}}_{n+r}(F)}\left(\mu^{(n+r)}\right)$, which is a degenerate principal series of $\widetilde{\mathrm{Sp}}_{n+r}(F)$. For an irreducible representation $\pi$ of $\widetilde{\mathrm{Sp}}_{r}(F)$, on which the kernel $\{ \pm 1\}$ of the covering map $\widetilde{\operatorname{Sp}_{r}}(F) \rightarrow \operatorname{Sp}_{r}(F)$ acts by $( \pm 1)^{n+r}$, the maximal $\pi$-isotypic quotient of $I_{\psi}^{(n+r)}(\tau)$ is of the form

$$
\mathcal{M}_{\psi, \tau}^{(n)}(\pi) \otimes \pi
$$

for some smooth representation $\mathcal{M}_{\psi, \tau}^{(n)}(\pi)$ of $\widetilde{\operatorname{Sp}}_{n}(F)$, on which the kernel $\{ \pm 1\}$ of the covering map $\widetilde{\operatorname{Sp}_{n}}(F) \rightarrow \operatorname{Sp}_{n}(F)$ acts by $( \pm 1)^{n+r}$. We call $\mathcal{M}_{\psi, \tau}^{(n)}(\pi)$ the local Miyawaki lift of $\pi$. 
3.2. Preliminary. In this subsection, we recall some basic terminologies of representations of $\widetilde{\mathrm{Sp}}_{r}(F)$.

A parabolic induction

$$
\tau_{1}|\cdot|^{s_{1}} \times \cdots \times \tau_{l}|\cdot|^{s_{l}} \rtimes \pi_{0}:=\operatorname{Ind}_{P(F)}^{\mathrm{Sp}_{r}(F)}\left(\tau_{1}|\cdot|^{s_{1}} \otimes \cdots \otimes \tau_{l}|\cdot|^{s_{l}} \otimes \pi_{0}\right),
$$

is standard if

- $P=M N$ is a standard parabolic subgroup of $\mathrm{Sp}_{r}$ with $M \cong \mathrm{GL}_{k_{1}} \times \cdots \times \mathrm{GL}_{k_{l}} \times \mathrm{Sp}_{r_{0}}$;

- $\tau_{i}\left(\right.$ resp. $\left.\pi_{0}\right)$ is an irreducible tempered representation of $\mathrm{GL}_{k_{i}}(F)\left(\operatorname{resp} . \mathrm{Sp}_{r_{0}}(F)\right)$;

- $s_{1}, \ldots, s_{l}$ are real numbers such that $s_{1}>\cdots>s_{l}>0$.

We understand that each irreducible tempered representation of $\operatorname{Sp}_{r}(F)$ is a standard module (the case where $l=0$ and $\left.r_{0}=r\right)$. The Langlands classification asserts that any irreducible smooth representation $\pi$ of $\operatorname{Sp}_{r}(F)$ is a unique irreducible quotient of a standard module $\tau_{1}|\cdot|{ }^{s_{1}} \times \cdots \times$ $\tau_{l}|\cdot|{ }^{s_{l}} \rtimes \pi_{0}$, which is called the Langlands quotient and is denoted by $J\left(\tau_{1}|\cdot|{ }^{s_{1}}, \ldots, \tau_{l}|\cdot|{ }^{s_{l}}, \pi_{0}\right)$. For any irreducible representation $\pi$, the datum $\left(P,\left\{\tau_{i}|\cdot|{ }^{s_{i}}\right\}, \pi_{0}\right)$ is determined uniquely up to an isomorphism.

When $\pi$ is a genuine irreducible representation of $\widetilde{\mathrm{Sp}}_{r}$, one should replace the parabolic induction with

$$
\operatorname{Ind}_{\widetilde{P}(F)}^{\widetilde{\mathrm{Sp}}_{r}(F)}\left(\tau_{1, \psi}|\cdot|^{s_{1}} \otimes \cdots \otimes \tau_{l, \psi}|\cdot|{ }^{s_{l}} \otimes \pi_{0}\right)
$$

where

$$
\tau_{i, \psi}(a, \zeta)=\zeta \frac{\alpha_{\psi}(1)}{\alpha_{\psi}(\operatorname{det} a)} \tau_{i}(a)
$$

is a genuine irreducible representation of the double cover $\widetilde{\mathrm{GL}}_{k_{i}}(F)$ of $\mathrm{GL}_{k_{i}}(F)$ (which is identified with $\mathrm{GL}_{k_{i}}(F) \times\{ \pm 1\}$ as sets), and $\pi_{0}$ is a genuine irreducible representation of $\widetilde{\operatorname{Sp}}_{r_{0}}(F)$. For more precision, see [15]. We identify $\tau_{\psi}$ with $\tau$ itself, and we use the same notation $\tau_{1}|\cdot|{ }^{s_{1}} \times \cdots \times \tau_{l} \mid \cdot{ }^{s_{l}} \rtimes \pi_{0}$ and $J\left(\tau_{1}|\cdot|{ }^{s_{1}}, \ldots, \tau_{l}|\cdot|^{s_{l}}, \pi_{0}\right)$ as in the non-genuine case.

We say that an irreducible representation $\pi$ of $\widetilde{\mathrm{Sp}_{r}}(F)$ is almost tempered if $\pi=J\left(\tau_{1}|\cdot|^{s_{1}}, \ldots, \tau_{l} \mid\right.$. $\left.\mid{ }^{s_{l}}, \pi_{0}\right)$ with $0<s_{l}<\cdots<s_{1}<1 / 2$. We understand that irreducible tempered representations are almost tempered (the case where $l=0$ ). Then the standard module $\tau_{1}|\cdot|{ }^{s_{1}} \times \cdots \times \tau_{l}|\cdot|{ }^{s_{l}} \rtimes \pi_{0}$ is irreducible by Corollary B.3, so that $\pi$ is equal to this standard module.

When we consider unramified representations, we always assume that the residue characteristic of $F$ is greater than 2. Then the covering map $\widetilde{\operatorname{Sp}_{r}}(F) \rightarrow \operatorname{Sp}_{r}(F)$ splits over the maximal compact subgroup $\operatorname{Sp}_{r}(\mathfrak{o})$, where $\mathfrak{o}$ is the ring of integers of $F$. Recall that an irreducible smooth representation $\pi$ of $\operatorname{Sp}_{r}(F)$ is unramified if $\pi$ has a nonzero $\operatorname{Sp}_{r}(\mathfrak{o})$-fixed vector. If $\pi$ is unramified, then there exist unramified characters $\chi_{1}, \ldots, \chi_{r}$ such that $\pi$ is a unique unramified constituent of the

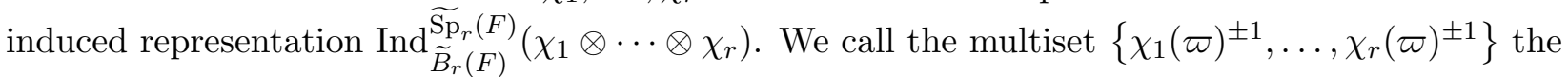
Satake parameter of $\pi$, where $\varpi$ is a uniformizer of $F$. We write $q=|\varpi|^{-1}$ for the cardinality of the residue field of $F$.

Recall that we can associate an irreducible representation $\pi$ of $\widetilde{\operatorname{Sp}_{r}}(F)$ to an $L$-parameter $\phi$, which is a self-dual representation of the Weil-Deligne group $W D_{F}=W_{F} \times \mathrm{SL}_{2}(\mathbb{C})$ of $F$. This is symplectic (resp. orthogonal) if $\pi$ is genuine (resp. not genuine). More precisely, see Appendix B. We say that an $L$-parameter $\phi$ is of good parity if $\phi$ is a sum of irreducible self-dual representations of the same type as $\phi$. The unique irreducible algebraic representation of $\mathrm{SL}_{2}(\mathbb{C})$ of dimension $d$ is denoted by $S_{d}$. 
3.3. Main local theorem. Recall that $\tau=\mu \times \mu^{-1}$ with $\mu$ being a unitary character of $F^{\times}$. Let $\chi_{-1}$ be the quadratic character associated to the extension $F(\sqrt{-1}) / F$. For a real number $a$, we denote by $[a]$ the maximal integer which is not greater than $a$. The determinant character of $\operatorname{GL}_{t}(F)$ is denoted by $\operatorname{det}_{t}$.

The following is the local main theorem.

Theorem 3.1. Let $\mu$ be a unitary character of $F^{\times}$, and $\pi$ be an irreducible representation of $\widetilde{\operatorname{Sp}_{r}}(F)$ on which $\{ \pm 1\}$ acts by $( \pm 1)^{n+r}$. Suppose that $n \geq r$.

(1) The local Miyawaki lift $\mathcal{M}_{\psi, \tau}^{(n)}(\pi)$ is nonzero and of finite length.

(2) If $\pi$ is almost tempered and unitary, then

- $\mathcal{M}_{\psi, \tau}^{(n)}(\pi)$ is irreducible;

- $\mathcal{M}_{\psi, \tau}^{(n)}(\pi) \cong\left(\mu^{\prime} \circ \operatorname{det}_{n-r}\right) \rtimes \pi$ with $\mu^{\prime}=\mu \chi_{-1}^{[(n+r) / 2]}$;

- $\mathcal{M}_{\psi, \tau}^{(n)}(\pi)$ is isomorphic to the unique irreducible quotient of the induced representation

$$
\begin{cases}\tau^{\prime}|\cdot|^{\frac{n-r-1}{2}} \times \tau^{\prime}|\cdot|^{\frac{n-r-3}{2}} \times \cdots \times \tau^{\prime}|\cdot|^{\frac{1}{2}} \rtimes \pi & \text { if } n+r \equiv 0 \bmod 2, \\ \tau^{\prime}|\cdot|^{\frac{n-r-1}{2}} \times \tau^{\prime}|\cdot|^{\frac{n-r-3}{2}} \times \cdots \times \tau^{\prime}|\cdot|^{1} \times \mu^{\prime} \rtimes \pi & \text { if } n+r \equiv 1 \bmod 2,\end{cases}
$$

where $\tau^{\prime}=\tau \otimes \chi_{-1}^{[(n+r) / 2]}=\mu^{\prime} \times \mu^{\prime-1}$.

(3) For any irreducible almost tempered unitary representations $\pi_{1}$ and $\pi_{2}$, we have

$$
\mathcal{M}_{\psi, \tau}^{(n)}\left(\pi_{1}\right) \cong \mathcal{M}_{\psi, \tau}^{(n)}\left(\pi_{2}\right) \Longrightarrow \pi_{1} \cong \pi_{2}
$$

(4) Suppose that $\pi$ is almost tempered and unitary so that $\mathcal{M}_{\psi, \tau}^{(n)}(\pi)$ is irreducible by (2). Assume further that one of the following conditions holds:

(a) The L-parameter $\phi$ does not contain $\mu^{ \pm 1} S_{d}$ for any $d \geq n-r$ with $d \equiv n-r$ mod 2 ;

(b) $n=r$ or $n=r+1$.

Set

$$
\pi^{\prime}=\mathcal{M}_{\psi, \tau}^{(r)}\left(\mathcal{M}_{\psi, \tau}^{(n)}(\pi)\right)
$$

Then all irreducible subquotients of $\pi^{\prime}$ are isomorphic to $\pi$, and the maximal semisimple quotient of $\pi^{\prime}$ is irreducible.

(5) Suppose that $\mu$ is unramified, and set $\alpha=\left(\mu \chi_{-1}^{[(n+r) / 2]}\right)(\varpi)$. If $\pi$ is an irreducible unramified representation of $\widetilde{\operatorname{Sp}}_{r}(F)$ with the Satake parameter $\left\{\beta_{1}^{ \pm 1}, \ldots, \beta_{r}^{ \pm 1}\right\}$, then $\mathcal{M}_{\psi, \tau}^{(n)}(\pi)$ has a unique irreducible unramified quotient. Its Satake parameter is equal to

$$
\left\{\beta_{1}^{ \pm 1}, \ldots, \beta_{r}^{ \pm 1}\right\} \cup\left\{\alpha^{ \pm 1} q^{\frac{n-r-1}{2}}, \alpha^{ \pm 1} q^{\frac{n-r-3}{2}}, \ldots, \alpha^{ \pm 1} q^{-\frac{n-r-1}{2}}\right\}
$$

as multisets.

As in Remark 3.9 (1) below, the condition (a) of Theorem 3.1 (4) holds when $n>2 r$ or $\pi$ is discrete series.

The assertion (2) gives the Langlands data for $\mathcal{M}_{\psi, \tau}^{(n)}(\pi)$ explicitly. In particular, it deduces (3). The proof of (5) for the non-genuine case (i.e., the case where $n+r$ is even) is [28, Proposition 3.1]. The genuine case is proven similarly.

We prove Theorem $3.1(1),(2)$ and (4) in $\S 3.5, \S 3.6$ and $\S 3.7$, respectively. 
3.4. Miyawaki liftings and degenerate induced representations. In this subsection, we show that $\mathcal{M}_{\psi, \tau}^{(n)}(\pi)$ is of finite length, and that for almost tempered $\pi$, there is a surjection

$$
\left(\mu^{\prime} \circ \operatorname{det}_{n-r}\right) \rtimes \pi \rightarrow \mathcal{M}_{\psi, \tau}^{(n)}(\pi) .
$$

We need the following lemma (see [36] and [16, Lemma 2.2]).

Lemma 3.2. Suppose that $n \geq r$. Set $\mu^{\prime}=\mu \chi_{-1}^{[(n+r) / 2]}$. The local Ikeda lift (the degenerate principal series) $I_{\psi}^{(n+r)}(\tau)=\operatorname{Ind}_{\widetilde{P}_{n+r}(F)}^{\widetilde{\mathrm{Sp}}_{n+r}(F)}\left(\mu^{(n+r)}\right)$ has an $\widetilde{\operatorname{Sp}_{n}}(F) \times \widetilde{\operatorname{Sp}_{r}}(F)$-equivariant filtration

$$
0 \subset I_{0} \subset I_{1} \subset \cdots \subset I_{r}=I_{\psi}^{(n+r)}(\tau)
$$

with successive quotients

$$
R_{t}=I_{t} / I_{t-1}=\operatorname{Ind}_{\widetilde{P}_{t+(n-r)}(F) \times \widetilde{\operatorname{Sp}}_{t}(F)} \underset{\widetilde{S p}_{r}(F)}{ }\left(\left(\mu^{\prime}\left|\operatorname{det}_{t+(n-r)}\right|^{\frac{t}{2}} \otimes \mu^{\prime}\left|\operatorname{det}_{t}\right|^{\frac{t+(n-r)}{2}}\right) \otimes C_{c}^{\infty}\left({\widetilde{\operatorname{Sp}_{r-t}}}_{r}(F)\right)\right) .
$$

Here the induction is normalized, and $\widetilde{\mathrm{Sp}}_{r-t}(F) \times{\widetilde{\mathrm{Sp}_{r-t}}}(F)$ acts on $C_{c}^{\infty}\left(\widetilde{\mathrm{Sp}}_{r-t}(F)\right)$ by

$$
\left(\left(g_{1}, g_{2}\right) \varphi\right)(x)=\varphi\left(g_{1}^{-1} \cdot x \cdot \alpha g_{2} \alpha^{-1}\right) \quad \text { with } \quad \alpha=\left(\begin{array}{cc}
\mathbf{1}_{r-t} & 0 \\
0 & -\mathbf{1}_{r-t}
\end{array}\right) \text {. }
$$

In particular,

$$
R_{0}=\operatorname{Ind}_{\widetilde{P}_{n-r}(F) \times \widetilde{\operatorname{Sp}}_{r}(F)}\left(\mu^{\prime} \circ \operatorname{det}_{n-r} \otimes C_{c}^{\infty}\left(\widetilde{\operatorname{Sp}}_{r}(F)\right)\right)
$$

Using this lemma, we have the following.

Proposition 3.3. Let $\pi$ and $\pi^{\prime}$ be smooth representations of $\widetilde{\operatorname{Sp}_{r}}(F)$ and $\widetilde{\operatorname{Sp}_{n}}(F)$, respectively. Set $\mu^{\prime}=\mu \chi_{-1}^{[(n+r) / 2]}$. Suppose that $\pi$ is irreducible, almost tempered and that

$$
\operatorname{Hom}_{\widetilde{\operatorname{Sp}}_{n}(F) \times \widetilde{\operatorname{Sp}}_{r}(F)}\left(I_{\psi}^{(n+r)}(\tau), \pi^{\prime} \otimes \pi\right) \neq 0 .
$$

Then we have

$$
\operatorname{Hom}_{\widetilde{\mathrm{Sp}}_{n}(F)}\left(\left(\mu^{\prime} \circ \operatorname{det}_{n-r}\right) \rtimes \pi, \pi^{\prime}\right) \neq 0 .
$$

Moreover, if there is an $\widetilde{\operatorname{Sp}_{n}}(F) \times \widetilde{\operatorname{Sp}_{r}}(F)$-equivariant surjection

$$
I_{\psi}^{(n+r)}(\tau) \rightarrow \pi^{\prime} \otimes \pi
$$

then there is an $\widetilde{\operatorname{Sp}_{n}}(F)$-equivariant surjection

$$
\left(\mu^{\prime} \circ \operatorname{det}_{n-r}\right) \rtimes \pi \rightarrow \pi^{\prime} .
$$

Proof. By Lemma 3.2, for some $0 \leq t \leq r$, we must have $\operatorname{Hom}_{\widetilde{\mathrm{Sp}_{n}}(F) \times \widetilde{\operatorname{Sp}_{r}}(F)}\left(R_{t}, \pi^{\prime} \otimes \pi\right) \neq 0$. By Bernstein's Frobenius reciprocity (see e.g., [9]), this Hom-space is isomorphic to the space of $\left(\widetilde{M}_{t+(n-r)}(F) \times \widetilde{M}_{t}(F)\right)$-equivariant maps

$$
\left(\mu^{\prime}\left|\operatorname{det}_{t+(n-r)}\right|^{\frac{t}{2}} \otimes \mu^{\prime}\left|\operatorname{det}_{t}\right|^{\frac{t+(n-r)}{2}}\right) \otimes C_{c}^{\infty}\left({\widetilde{\operatorname{Sp}_{r-t}}}(F)\right) \rightarrow R_{\overline{P_{t+(n-r)}(F)}}\left(\pi^{\prime}\right) \otimes R_{\overline{P_{t}(F)}}(\pi),
$$

where $R_{\overline{P_{t}(F)}}(\pi)$ is the normalized Jacquet module of $\pi$ with respect to (the double cover of) the opposite parabolic subgroup $\overline{P_{t}(F)}$ to $P_{t}(F)$. First, we assume that $t>0$. By taking the contragredient, we have

$$
\operatorname{Hom}_{\widetilde{\mathrm{GL}}_{t}(F)}\left(R_{P_{t}(F)}\left(\pi^{\vee}\right), \mu^{\prime-1}\left|\operatorname{det}_{t}\right|^{\frac{-t-(n-r)}{2}}\right) \neq 0 .
$$


Since $\pi^{\vee}$ is almost tempered, we must have $-t-(n-r)>-1$. This contradicts $n \geq r$ and $t>0$. Hence we must have $t=0$, and

$$
\operatorname{Hom}_{\widetilde{M}_{n-r}(F) \times \widetilde{\operatorname{Sp}}_{r}(F)}\left(\mu^{\prime} \circ \operatorname{det}_{n-r} \otimes C_{c}^{\infty}\left(\widetilde{\operatorname{Sp}_{r}}(F)\right), R_{\overline{P_{n-r}(F)}}\left(\pi^{\prime}\right) \otimes \pi\right) \neq 0 .
$$

Taking care of the action of $\widetilde{\operatorname{Sp}}_{r}(F) \times \widetilde{\operatorname{Sp}}_{r}(F)$ on $C_{c}^{\infty}\left(\widetilde{\operatorname{Sp}}_{r}(F)\right)$, we see that this Hom-space is isomorphic to

$$
\operatorname{Hom}_{\widetilde{M}_{n-r}(F)}\left(\mu^{\prime} \circ \operatorname{det}_{n-r} \otimes \pi, R_{\overline{P_{n-r}(F)}}\left(\pi^{\prime}\right)\right) .
$$

Using Bernstein's Frobenius reciprocity, we obtain the first assertion.

Suppose that there is an $\widetilde{\operatorname{Sp}_{n}}(F) \times \widetilde{\operatorname{Sp}}_{r}(F)$-equivariant surjection $I_{r} \rightarrow \pi^{\prime} \otimes \pi$. Then the image of $I_{0}$ is of the form $\pi_{0}^{\prime} \otimes \pi$ for some $\widetilde{\operatorname{Sp}}_{n}(F)$-subrepresentation $\pi_{0}^{\prime}$ of $\pi^{\prime}$, and it induces a surjection $I_{r} / I_{0} \rightarrow\left(\pi^{\prime} / \pi_{0}^{\prime}\right) \otimes \pi$. The above argument implies $\left(\pi^{\prime} / \pi_{0}^{\prime}\right)=0$ so that the restriction gives an $\widetilde{\operatorname{Sp}}_{n}(F) \times \widetilde{\operatorname{Sp}_{r}}(F)$-equivariant surjection $I_{0} \rightarrow \pi^{\prime} \otimes \pi$. This induces an $\widetilde{\operatorname{Sp}_{n}}(F)$-equivariant surjection $\left(\mu^{\prime} \circ \operatorname{det}_{n-r}\right) \rtimes \pi \rightarrow \pi^{\prime}$.

Let $\pi$ and $\pi^{\prime}$ be smooth representations of $\widetilde{\operatorname{Sp}_{r}}(F)$ and $\widetilde{\operatorname{Sp}_{n}}(F)$, respectively. Suppose that $\pi$ is irreducible, and that there exists a surjection

$$
I_{\psi}^{(n+r)}(\tau) \rightarrow \pi^{\prime} \otimes \pi
$$

Then Lemma 3.2 gives a filtration

$$
0 \subset \pi_{0}^{\prime} \subset \pi_{1}^{\prime} \subset \cdots \subset \pi_{r}^{\prime}=\pi^{\prime} .
$$

More precisely, the restriction of the surjection to $I_{t} \subset I_{\psi}^{(n+r)}(\tau)$ defines a subrepresentation $\pi_{t}^{\prime}$ of $\pi^{\prime}$ so that the image of $I_{t}$ is $\pi_{t}^{\prime} \otimes \pi$. By a similar argument to Proposition 3.3, one can show that the successive quotients $\pi_{t}^{\prime} / \pi_{t-1}^{\prime}$ are of finite length. Therefore $\pi^{\prime}$ must also be of finite length.

3.5. Non-vanishing of local Miyawaki liftings. Next, we show Theorem 3.1 (1). We have seen that $\mathcal{M}_{\psi, \tau}^{(n)}(\pi)$ is of finite length. We show that $\mathcal{M}_{\psi, \tau}^{(n)}(\pi) \neq 0$. In fact, we will prove that

$$
\operatorname{Hom}_{\widetilde{\operatorname{Sp}}_{n}(F) \times \widetilde{\mathrm{Sp}}_{r}(F)}\left(I_{\psi}^{(n+r)}(\tau),\left(\left(\mu^{\prime} \circ \operatorname{det}_{n-r}\right) \rtimes \pi\right) \otimes \pi\right) \neq 0
$$

for any irreducible representation $\pi$ of $\widetilde{\mathrm{Sp}_{r}}(F)$.

Using

$$
\operatorname{Sp}_{2 r}(F) \hookrightarrow \operatorname{Sp}_{n+r}(F),\left(\begin{array}{cc}
A & B \\
C & D
\end{array}\right) \mapsto\left(\begin{array}{ll|ll}
\mathbf{1}_{n-r} & & & \\
& A & & B \\
\hline & C & \mathbf{1}_{n-r} & \\
& & D
\end{array}\right),
$$

we regard $\widetilde{\operatorname{Sp}}_{2 r}(F)$ as a subgroup of $\widetilde{\operatorname{Sp}}_{n+r}(F)$. Then for $\Phi \in I_{\psi}^{(n+r)}(\tau)$, we have

$$
\left.\Phi\right|_{\widetilde{\operatorname{Sp}}_{2 r}(F)} \in \operatorname{Ind}_{\widetilde{P}_{2 r}(F)}^{\widetilde{\mathrm{Sp}}_{2 r}(F)}\left(\mu \chi_{-1}^{\left[\frac{n+r}{2}\right]}\left|\operatorname{det}_{2 r}\right|^{\frac{n-r}{2}}\right)
$$

By the theory of the doubling method [38], [43], a doubling zeta integral gives a nonzero element

$$
\mathcal{Z}_{r, r} \in \operatorname{Hom}_{\widetilde{\operatorname{Sp}_{r}}(F) \times \widetilde{\operatorname{Sp}}_{r}(F)}\left(\operatorname{Ind}_{\widetilde{P}_{2 r}(F)}^{\widetilde{\operatorname{Sp}}_{2 r}(F)}\left(\mu \chi_{-1}^{\left[\frac{n+r}{2}\right]}\left|\operatorname{det}_{2 r}\right|^{\frac{n-r}{2}}\right) \otimes(\pi \otimes \pi), \mathbb{C}\right) .
$$


Note that the embedding $\iota: \mathrm{Sp}_{r}(F) \times \mathrm{Sp}_{r}(F) \hookrightarrow \mathrm{Sp}_{2 r}(F)$ is not the usual one in this theory (see $[38])$. For $\Phi \in I_{\psi}^{(n+r)}(\tau), f \in\left(\mu^{-1} \chi_{-1}^{[-(n+r) / 2]} \circ \operatorname{det}_{n-r}\right) \rtimes \pi$, and $v \in \pi$, we consider the integral

$$
\mathcal{Z}_{r, n}(\Phi, f, v)=\int_{P_{n-r}(F) \backslash \operatorname{Sp}_{n}(F)} \mathcal{Z}_{r, r}\left(\left.(\iota(g, 1), \zeta) \Phi\right|_{\widetilde{S p}_{2 r}(F)} \otimes f(g, \zeta) \otimes v\right) d g .
$$

Proposition 3.4. The integral $\mathcal{Z}_{r, n}(\Phi, f, v)$ is well-defined and gives a nonzero element in

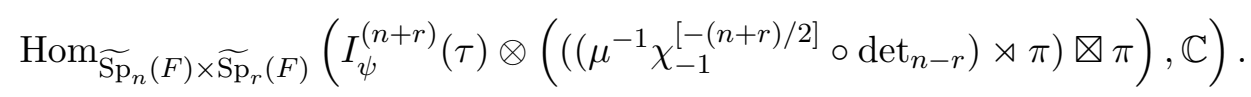

Proof. Since

$$
h \cdot \iota(u, 1) \cdot h^{-1}=\left(\begin{array}{cc|cc}
\mathbf{1}_{n-r} & * & * & * \\
0 & \mathbf{1}_{2 r} & * & 0 \\
\hline 0 & 0 & \mathbf{1}_{n-r} & 0 \\
0 & 0 & * & \mathbf{1}_{2 r}
\end{array}\right)
$$

for $u \in N_{n-r}(F) \subset \operatorname{Sp}_{n}(F)$ and $h \in{\widetilde{\operatorname{Sp}_{2 r}}}(F)$, we have $\Phi(h \cdot(\iota(u g, 1), \zeta))=\Phi(h \cdot(\iota(g, 1), \zeta))$. Similarly, we have

$$
\begin{aligned}
& \Phi\left(h \cdot\left(\iota\left(\left(\begin{array}{cc|cc}
a & 0 & 0 & 0 \\
0 & \mathbf{1}_{r} & 0 & 0 \\
\hline 0 & 0 & { }^{t} a^{-1} & 0 \\
0 & 0 & 0 & \mathbf{1}_{r}
\end{array}\right) g, 1\right), \zeta\right)\right) \otimes f\left(\left(\begin{array}{cc|cc}
a & 0 & 0 & 0 \\
0 & \mathbf{1}_{r} & 0 & 0 \\
\hline 0 & 0 & { }^{t} a^{-1} & 0 \\
0 & 0 & 0 & \mathbf{1}_{r}
\end{array}\right) g, \zeta\right) \\
& =|\operatorname{det} a|^{\frac{n+r+1}{2}} \Phi(h \cdot(\iota(g, 1), \zeta)) \otimes f(g, \zeta)
\end{aligned}
$$

for $a \in \mathrm{GL}_{n-r}(F)$. Hence we have

$$
\begin{aligned}
& \mathcal{Z}_{r, r}\left(\left.(\iota(p g, 1), \zeta) \Phi\right|_{\widetilde{\operatorname{Sp}}_{2 r}(F)} \otimes f(p g, \zeta) \otimes v\right) \\
& =\delta_{P_{n-r}}(p) \cdot \mathcal{Z}_{r, r}\left(\left.(\iota(g, 1), \zeta) \Phi\right|_{\widetilde{S p}_{2 r}(F)} \otimes f(g, \zeta) \otimes v\right)
\end{aligned}
$$

for $p \in P_{n-r}(F) \subset \operatorname{Sp}_{n}(F)$, where $\delta_{P_{n-r}}$ is the modulus character of $P_{n-r}(F)$. This shows that $\mathcal{Z}_{r, n}(\Phi, f, v)$ is well-defined.

It is easy to see that

$$
\mathcal{Z}_{r, n} \in \operatorname{Hom}_{\widetilde{\operatorname{Sp}_{n}}(F) \times \widetilde{\operatorname{Sp}}_{r}(F)}\left(I_{\psi}^{(n+r)}(\tau) \otimes\left(\left(\left(\mu^{-1} \chi_{-1}^{[-(n+r) / 2]} \circ \operatorname{det}_{n-r}\right) \rtimes \pi\right) \otimes \pi\right), \mathbb{C}\right) .
$$

Since

$$
I_{\psi}^{(n+r)}(\tau) \rightarrow \operatorname{Ind}_{\widetilde{P}_{2 r}(F)}^{\widetilde{\operatorname{Sp}}_{2 r}(F)}\left(\mu \chi_{-1}^{\left[\frac{n+r}{2}\right]}\left|\operatorname{det}_{2 r}\right|^{\frac{n-r}{2}}\right),\left.\Phi \mapsto \Phi\right|_{\widetilde{S p}_{2 r}(F)}
$$

is surjective, we see that $\mathcal{Z}_{r, n} \neq 0$.

Applying Proposition 3.4 for $\pi^{\vee}$, we conclude that

$$
\operatorname{Hom}_{\widetilde{\operatorname{Sp}_{n}}(F) \times \widetilde{\operatorname{Sp}}_{r}(F)}\left(I_{\psi}^{(n+r)}(\tau),\left(\left(\mu^{\prime} \circ \operatorname{det}_{n-r}\right) \rtimes \pi\right) \otimes \pi\right) \neq 0
$$

since $\left(\left(\mu^{\prime} \circ \operatorname{det}_{n-r}\right) \rtimes \pi\right)^{\vee} \cong\left(\mu^{-1} \chi_{-1}^{[-(n+r) / 2]} \circ \operatorname{det}_{n-r}\right) \rtimes \pi^{\vee}$. 
3.6. Irreducibility of degenerate induced representations. To show Theorem 3.1 (2), we need to prove the irreducibility of the induced representation $\left(\mu \circ \operatorname{det}_{n-r}\right) \rtimes \pi$, where $\pi$ is an irreducible almost tempered unitary representation of $\widetilde{\operatorname{Sp}}_{r}(F)$, and $\mu$ is a unitary character of $F^{\times}$. When $\pi$ is supercuspidal and $\delta=0$, the irreducibility of $\left(\mu \circ \operatorname{det}_{n-r}\right) \rtimes \pi$ was proven by Tadic $[52$, Theorem 9.1], and its Langlands data was given by Jantzen [31]. We imitate their proofs.

We first show the irreducibility of other induced representations. Let $\pi$ be an irreducible representation of $\widetilde{\operatorname{Sp}}_{r}(F)$. We set $\delta=1$ if $\pi$ is genuine, and $\delta=0$ otherwise. For a smooth representation $\Pi$ of $\widetilde{\operatorname{Sp}}_{n}(F)$, we write s.s. $(\Pi)$ for the semisimplification of $\Pi$.

Proposition 3.5. Let $\mu$ be a unitary character of $F^{\times}$, and $\pi$ be an irreducible tempered representation of $\widetilde{\operatorname{Sp}}_{r}(F)$. Suppose that the L-parameter $\phi$ for $\pi$ is of good parity (see §3.2). Then for $l \geq k-\delta$ with $l \equiv k-\delta \bmod 2$, the induced representation

$$
\mu^{-1}\left|\operatorname{det}_{k}\right|^{\frac{l}{2}} \rtimes \pi
$$

is irreducible.

Proof. We prove the proposition by induction on $k$. When $k=1$ and $l>0$, this is Corollary B.4. When $k=1$ and $l=0$ so that $\delta=1$, the irreducibility of $\mu^{-1} \rtimes \pi$ follows from Theorem B.1 (6).

Suppose that $k>1$ and that $\mu^{-1}\left|\operatorname{det}_{k}\right|^{\frac{l}{2}} \rtimes \pi$ is reducible. Let $\sigma_{1}, \ldots, \sigma_{t}$ be irreducible representations of ${\widetilde{\operatorname{Sp}_{k+r}}}_{k}(F)$ such that s.s. $\left(\mu^{-1}\left|\operatorname{det}_{k}\right|^{\frac{l}{2}} \rtimes \pi\right)=\sigma_{1} \oplus \cdots \oplus \sigma_{t}$, and that $\sigma_{1}$ is a submodule and $\sigma_{t}$ is a quotient of $\mu^{-1}\left|\operatorname{det}_{k}\right|^{\frac{l}{2}} \rtimes \pi$ :

$$
\sigma_{1} \hookrightarrow \mu^{-1}\left|\operatorname{det}_{k}\right|^{\frac{l}{2}} \rtimes \pi \rightarrow \sigma_{t} .
$$

Since the Langlands quotient appears in the standard module with multiplicity one as a subquotients, we have $\sigma_{i} \not \sigma_{t}$ for $i \neq t$.

By Proposition A.4, we have

$$
\begin{aligned}
\text { s.s. } R_{P_{1}(F)}\left(\mu^{-1}\left|\operatorname{det}_{k}\right|^{\frac{l}{2}} \rtimes \pi\right) & =\mu|\cdot|^{-\frac{l+k-1}{2}} \otimes\left(\mu^{-1}\left|\operatorname{det}_{k-1}\right|^{\frac{l-1}{2}} \rtimes \pi\right) \\
& \oplus \mu^{-1}|\cdot|^{\frac{l-k+1}{2}} \otimes\left(\mu^{-1}\left|\operatorname{det}_{k-1}\right|^{\frac{l+1}{2}} \rtimes \pi\right) \\
& \oplus \text { s.s. }\left(\bigoplus_{\lambda} \chi_{\lambda}|\cdot|^{\alpha_{\lambda}} \otimes\left(\mu^{-1}\left|\operatorname{det}_{k}\right|^{\frac{l}{2}} \rtimes \pi_{\lambda}\right)\right),
\end{aligned}
$$

where s.s. $R_{P_{1}(F)}(\pi)=\oplus_{\lambda} \chi_{\lambda}|\cdot|^{\alpha_{\lambda}} \nabla \pi_{\lambda}$ with $\chi_{\lambda}$ unitary and $\alpha_{\lambda} \in \mathbb{R}$. By the induction hypothesis, we see that $\mu^{-1}\left|\operatorname{det}_{k-1}\right|^{(l \pm 1) / 2} \rtimes \pi$ is irreducible. By Corollary B.5, we have $2 \alpha_{\lambda} \in \mathbb{Z}$ and $2 \alpha_{\lambda} \equiv$ $l-k \bmod 2$. In particular, the first two summands appear in s.s. $R_{P_{1}(F)}\left(\mu^{-1}\left|\operatorname{det}_{k}\right|^{\frac{l}{2}} \rtimes \pi\right)$ with multiplicity one.

Since $\sigma_{1} \hookrightarrow \mu^{-1}\left|\operatorname{det}_{k}\right|^{\frac{l}{2}} \rtimes \pi$ and $\sigma_{t} \hookrightarrow \mu\left|\operatorname{det}_{k}\right|^{-\frac{l}{2}} \rtimes \pi$, we have

$$
\begin{aligned}
& \text { s.s. } R_{P_{1}(F)}\left(\sigma_{i}\right) \supset \mu^{-1}|\cdot|^{\frac{l-k+1}{2}} \otimes\left(\mu^{-1}\left|\operatorname{det}_{k-1}\right|^{\frac{l+1}{2}} \rtimes \pi\right) \Longleftrightarrow i=1, \\
& \text { s.s. } R_{P_{1}(F)}\left(\sigma_{i}\right) \supset \mu|\cdot|^{-\frac{l+k-1}{2}} \otimes\left(\mu^{-1}\left|\operatorname{det}_{k-1}\right|^{\frac{l-1}{2}} \rtimes \pi\right) \Longleftrightarrow i=t .
\end{aligned}
$$

On the other hand, by Proposition A.4, we have

$$
\text { s.s. } R_{P_{k}(F)}\left(\mu^{-1}\left|\operatorname{det}_{k}\right|^{\frac{l}{2}} \rtimes \pi\right) \supset \text { s.s. }\left(\mu\left|\operatorname{det}_{k-1}\right|^{-\frac{l+1}{2}} \times \mu^{-1}|\cdot|^{\frac{l-k+1}{2}}\right) \otimes \pi \text {. }
$$

Note that $\mu\left|\operatorname{det}_{k-1}\right|^{-\frac{l+1}{2}}=\langle\mu ;-(l+k-1) / 2, \ldots,(-l+k-3) / 2\rangle$ and $\mu^{-1}|\cdot|^{\frac{l-k+1}{2}}=\left\langle\mu^{-1} ;(l-k+1) / 2\right\rangle$ in the notation in Appendix A.1. Unless $\delta=1, l=k-1$ and $\mu^{-1}=\mu$, the representation 
$\mu\left|\operatorname{det}_{k-1}\right|^{-\frac{l+1}{2}} \times \mu^{-1}|\cdot| \frac{l-k+1}{2}$ is irreducible by Theorem A.2. When it is irreducible, there exists $i$ such that

$$
\text { s.s. } R_{P_{k}(F)}\left(\sigma_{i}\right) \supset\left(\mu\left|\operatorname{det}_{k-1}\right|^{-\frac{l+1}{2}} \times \mu^{-1}|\cdot|^{\frac{l-k+1}{2}}\right) \otimes \pi .
$$

Considering the Jacquet module with respect to $P_{k} \cap P_{1}$, we see that

$$
\text { s.s. } R_{P_{1}(F)}\left(\sigma_{i}\right) \supset \mu^{-1}|\cdot|^{\frac{l-k+1}{2}} \otimes\left(\mu^{-1}\left|\operatorname{det}_{k-1}\right|^{\frac{l+1}{2}} \rtimes \pi\right) \oplus \mu|\cdot|^{-\frac{l+k-1}{2}} \otimes\left(\mu^{-1}\left|\operatorname{det}_{k-1}\right|^{\frac{l-1}{2}} \rtimes \pi\right) .
$$

This implies that $i=1$ and $i=t$. We obtain a contradiction.

Similarly, when $k \geq 3$, we have

$$
\text { s.s. } R_{P_{k}(F)}\left(\mu^{-1}\left|\operatorname{det}_{k}\right|^{\frac{l}{2}} \rtimes \pi\right) \supset\left(\mu\left|\operatorname{det}_{k-2}\right|^{-\frac{l+2}{2}} \times \mu^{-1}\left|\operatorname{det}_{2}\right|^{\frac{l-k+2}{2}}\right) \otimes \pi \text {. }
$$

The right hand side is irreducible by Theorem A.2 since $\mu\left|\operatorname{det}_{k-2}\right|^{-\frac{l+2}{2}}=\langle\mu ;-(l+k-1) / 2, \ldots,(-l+$ $k-5) / 2\rangle$ and $\mu^{-1}\left|\operatorname{det}_{2}\right|^{\frac{l-k+2}{2}}=\left\langle\mu^{-1} ;(l-k+1) / 2,(l-k+3) / 2\right\rangle$. By the same argument, we obtain a contradiction.

Therefore, the proposition is reduced to the case where $\delta=1, k=l+1=2$ and $\mu^{-1}=\mu$. We treat this case in the following lemma.

Lemma 3.6. Let $\pi$ be an irreducible genuine tempered representation of $\widetilde{\operatorname{Sp}}_{r}(F)$ so that $\delta=1$. Suppose that $\mu$ is a quadratic character, and that the L-parameter $\phi$ for $\pi$ is of good parity. Then the induced representation

$$
\mu\left|\operatorname{det}_{2}\right|^{\frac{1}{2}} \rtimes \pi
$$

is irreducible.

Proof. First, we show that $\left(\mu \circ \operatorname{det}_{3}\right) \rtimes \pi$ is irreducible in this case. By Theorem A.2 and Corollary B.4, we have

$$
\begin{aligned}
\tau|\cdot| \times \mu \rtimes \pi & \cong \mu|\cdot| \times \mu|\cdot| \times \mu \rtimes \pi \\
& \rightarrow \mu|\cdot| \times \mu\left|\operatorname{det}_{2}\right|^{\frac{1}{2}} \rtimes \pi \\
& \cong \mu\left|\operatorname{det}_{2}\right|^{\frac{1}{2}} \times \mu|\cdot| \rtimes \pi \\
& \cong \mu\left|\operatorname{det}_{2}\right|^{\frac{1}{2}} \times \mu|\cdot|^{-1} \rtimes \pi \\
& \rightarrow\left(\mu \circ \operatorname{det}_{3}\right) \rtimes \pi .
\end{aligned}
$$

Since $\pi$ is unitary, $\left(\mu \circ \operatorname{det}_{3}\right) \rtimes \pi$ is semisimple. Therefore we deduce that $\left(\mu \circ \operatorname{det}_{3}\right) \rtimes \pi \cong J(\tau|\cdot|, \mu \rtimes \pi)$.

Now we start to prove the lemma. Suppose for the sake of contradiction that $\mu\left|\operatorname{det}_{2}\right|^{\frac{1}{2}} \rtimes \pi$ is reducible. Take an irreducible submodule of $\sigma$, and an irreducible quotient $\sigma^{\prime}$ of $\mu\left|\operatorname{det}_{2}\right|^{\frac{1}{2}} \rtimes \pi$ :

$$
\sigma \hookrightarrow \mu\left|\operatorname{det}_{2}\right|^{\frac{1}{2}} \rtimes \pi \rightarrow \sigma^{\prime} .
$$

Then $\sigma \neq \sigma^{\prime}$. Moreover, we have

$$
\mu|\cdot|^{1} \rtimes \sigma \hookrightarrow \mu|\cdot|^{1} \times \mu\left|\operatorname{det}_{2}\right|^{\frac{1}{2}} \rtimes \pi \rightarrow \mu|\cdot|^{1} \rtimes \sigma^{\prime} .
$$

Since $\tau|\cdot| \times \mu \rtimes \pi \rightarrow \mu|\cdot|^{1} \times \mu\left|\operatorname{det}_{2}\right|^{\frac{1}{2}} \rtimes \pi$, we have $\mu|\cdot|{ }^{1} \rtimes \sigma^{\prime} \rightarrow\left(\mu \circ \operatorname{det}_{3}\right) \rtimes \pi$. Moreover, since any standard module has its Langlands quotient with multiplicity one as a subquotient, we have

$$
\left(\mu \circ \operatorname{det}_{3}\right) \rtimes \pi \not \subset \text { s.s. }\left(\mu|\cdot|^{1} \rtimes \sigma\right) \text {. }
$$


Using proposition A.4, we compute the Jacquet modules s.s. $R_{P_{1}(F)}\left(\mu|\cdot|{ }^{1} \times \mu\left|\operatorname{det}_{2}\right|^{\frac{1}{2}} \rtimes \pi\right)$ and s.s. $R_{P_{1}(F)}\left(\left(\mu \circ \operatorname{det}_{3}\right) \rtimes \pi\right)$. Then the sums of all irreducible representations of the form $\mu|\cdot|^{-1} \otimes \Sigma$ which appear in s.s. $R_{P_{1}(F)}\left(\mu|\cdot|^{1} \times \mu\left|\operatorname{det}_{2}\right|^{\frac{1}{2}} \rtimes \pi\right)$ and s.s. $R_{P_{1}(F)}\left(\left(\mu \circ \operatorname{det}_{3}\right) \rtimes \pi\right)$ are isomorphic to

$$
\text { s.s. }\left(\mu|\cdot|^{-1} \otimes \mu\left|\operatorname{det}_{2}\right|^{\frac{1}{2}} \rtimes \pi\right) \oplus \text { s.s. }\left(\mu|\cdot|^{-1} \bigotimes\left(\mu|\cdot|^{1} \times \mu\right) \rtimes \pi\right)
$$

and

$$
\text { s.s. }\left(\mu|\cdot|^{-1} \otimes \mu\left|\operatorname{det}_{2}\right|^{-\frac{1}{2}} \rtimes \pi\right) \oplus \text { s.s. }\left(\mu|\cdot|^{-1} \otimes \mu\left|\operatorname{det}_{2}\right|^{\frac{1}{2}} \rtimes \pi\right),
$$

respectively. Hence the difference is

$$
\mu|\cdot|^{-1} \otimes \mu|\cdot|^{\frac{1}{2}} \mathrm{St}_{2} \rtimes \pi,
$$

where $\mu|\cdot|^{\frac{1}{2}} \mathrm{St}_{2}=\langle\mu ; 1,0\rangle$ is a Steinberg representation. By Proposition B.2, we see that $\mu|\cdot| \frac{1}{2} \mathrm{St}_{2} \rtimes \pi$ is irreducible. Since

$$
\text { s.s. } R_{P_{1}(F)}\left(\mu|\cdot|^{1} \rtimes \sigma\right) \supset \mu|\cdot|^{-1} \bigotimes \sigma,
$$

we deduce that $\sigma=\mu|\cdot|^{\frac{1}{2}} \mathrm{St}_{2} \rtimes \pi$. However, we have

$$
\text { s.s. } R_{P_{1}(F)}\left(\mu|\cdot|^{\frac{1}{2}} \mathrm{St}_{2} \rtimes \pi\right) \supset \mu|\cdot|^{1} \bigotimes \mu \rtimes \pi \not \subset \text { s.s. } R_{P_{1}(F)}\left(\mu\left|\operatorname{det}_{2}\right|^{\frac{1}{2}} \rtimes \pi\right) .
$$

Therefore, $\mu|\cdot|^{\frac{1}{2}} \mathrm{St}_{2} \rtimes \pi \not \subset \mu\left|\operatorname{det}_{2}\right|^{\frac{1}{2}} \rtimes \pi$, which is a contradiction.

Now we show the following.

Theorem 3.7. Let $\pi$ be an irreducible almost tempered representation of $\widetilde{\operatorname{Sp}}_{r}(F)$, and $\mu$ be a unitary character of $F^{\times}$. Set $\tau=\mu \times \mu^{-1}$. Then there exists a surjection

$$
\left\{\begin{aligned}
\tau|\cdot|^{k-\frac{1}{2}} \times \tau|\cdot|^{k-\frac{3}{2}} \times \cdots \times \tau|\cdot|^{\frac{1}{2}} \rtimes \pi \rightarrow\left(\mu \circ \operatorname{det}_{2 k}\right) \rtimes \pi & \text { if } \delta=0, \\
\left.\tau|\cdot|\right|^{k-1} \times\left.\tau|\cdot|\right|^{k-2} \times \cdots \times \tau|\cdot|^{1} \times \mu \rtimes \pi \rightarrow\left(\mu \circ \operatorname{det}_{2 k-1}\right) \rtimes \pi & \text { if } \delta=1 .
\end{aligned}\right.
$$

In particular, if $\pi$ is unitary, then $\left(\mu \circ \operatorname{det}_{2 k-\delta}\right) \rtimes \pi$ is irreducible.

Proof. By Theorem B.1, we have

$$
\pi \cong \tau_{1} \times \cdots \times \tau_{l} \rtimes \pi_{0}
$$

where

- $\tau_{i}=|\cdot|^{s_{i}} \tau_{i}^{\prime}$ with $\tau_{i}^{\prime}$ being an irreducible discrete series representation of $\mathrm{GL}_{k_{i}}(F)$;

- $1 / 2>s_{1} \geq s_{2} \geq \cdots \geq s_{l} \geq 0$

- when $s_{i}=0$, the irreducible representation $\phi_{i}$ of $W D_{F}$ corresponding to $\tau_{i}$ is not orthogonal if $\delta=0$, and is not symplectic if $\delta=1$;

- $\pi_{0}$ is an irreducible representation of $\widetilde{\mathrm{Sp}}_{r_{0}}(F)$ whose $L$-parameter is of good parity.

The segments corresponding to $\mu^{-1}\left|\operatorname{det}_{k}\right|^{\frac{k-\delta}{2}}$ and $\mu\left|\operatorname{det}_{k}\right|^{-\frac{k-\delta}{2}}$ are $[(1-\delta) / 2, k-(1+\delta) / 2]$ and $[-k+(1+\delta) / 2,-(1-\delta) / 2]$, respectively, which contain $(1-\delta) / 2$ or $-(1-\delta) / 2$. Hence by Theorem A.2, we see that

$$
\begin{aligned}
\mu^{-1}\left|\operatorname{det}_{k}\right|^{\frac{k-\delta}{2}} \times \tau_{i} & \cong \tau_{i} \times \mu^{-1}\left|\operatorname{det}_{k}\right|^{\frac{k-\delta}{2}} \\
\mu\left|\operatorname{det}_{k}\right|^{-\frac{k-\delta}{2}} \times \tau_{i} & \cong \tau_{i} \times \mu\left|\operatorname{det}_{k}\right|^{-\frac{k-\delta}{2}}
\end{aligned}
$$

By Proposition 3.5, we have

$$
\mu^{-1}\left|\operatorname{det}_{k}\right|^{\frac{k-\delta}{2}} \rtimes \pi_{0} \cong \mu\left|\operatorname{det}_{k}\right|^{-\frac{k-\delta}{2}} \rtimes \pi_{0} .
$$


Therefore,

$$
\begin{aligned}
& \left.\tau|\cdot|\right|^{k-\frac{1+\delta}{2}} \times\left.\tau|\cdot|\right|^{k-\frac{1+\delta}{2}-1} \times \cdots \times \tau|\cdot|^{1+\delta} \times\left(\mu \circ \operatorname{det}_{\delta}\right) \rtimes \pi \\
& \rightarrow \mu\left|\operatorname{det}_{k-\delta}\right|^{\frac{k}{2}} \times \mu^{-1}\left|\operatorname{det}_{k}\right|^{\frac{k-\delta}{2}} \rtimes \pi \\
& \cong \mu\left|\operatorname{det}_{k-\delta}\right|^{\frac{k}{2}} \times \tau_{1} \times \cdots \times \tau_{l} \times \mu^{-1}\left|\operatorname{det}_{k}\right|^{\frac{k-\delta}{2}} \rtimes \pi_{0} \\
& \cong \mu\left|\operatorname{det}_{k-\delta}\right|^{\frac{k}{2}} \times \tau_{1} \times \cdots \times \tau_{l} \times \mu\left|\operatorname{det}_{k}\right|^{-\frac{k-\delta}{2}} \rtimes \pi_{0} \\
& \cong \mu\left|\operatorname{det}_{k-\delta}\right|^{\frac{k}{2}} \times \mu\left|\operatorname{det}_{k}\right|^{-\frac{k-\delta}{2}} \rtimes \pi \\
& \rightarrow\left(\mu \circ \operatorname{det}_{2 k-\delta}\right) \rtimes \pi .
\end{aligned}
$$

If $\pi$ is unitary, then $\left(\mu \circ \operatorname{det}_{2 k-\delta}\right) \rtimes \pi$ is semisimple. Therefore, by the uniqueness of the Langlands quotient, we conclude that $\left(\mu \circ \operatorname{det}_{2 k-\delta}\right) \rtimes \pi$ is irreducible.

This theorem together with Proposition 3.3 implies Theorem 3.1 (2).

3.7. Going down case. Finally, we show Theorem 3.1 (4).

Let $\pi$ be an irreducible almost tempered unitary representation of $\widetilde{\operatorname{Sp}}_{r}(F)$. Note that $\mathcal{M}_{\psi, \tau}^{(n)}(\pi)$ is irreducible by Theorem 3.1 (2). Set

$$
\pi^{\prime}=\mathcal{M}_{\psi, \tau}^{(r)}\left(\mathcal{M}_{\psi, \tau}^{(n)}(\pi)\right)
$$

Since there is a surjection $\pi^{\prime} \rightarrow \pi$, we have $\pi^{\prime} \neq 0$. Moreover, by the definition and Theorem 3.1 (2), we obtain a surjection

$$
I_{\psi}^{(n+r)}(\tau) \rightarrow\left(\left(\mu^{\prime} \circ \operatorname{det}_{n-r}\right) \rtimes \pi\right) \otimes \pi^{\prime} .
$$

By Lemma 3.2, there is $0 \leq t \leq r$ such that $\operatorname{Hom}_{\widetilde{\operatorname{Sp}_{n}}(F) \times \widetilde{\operatorname{Sp}_{r}}(F)}\left(R_{t},\left(\left(\mu^{\prime} \circ \operatorname{det}_{n-r}\right) \rtimes \pi\right) \otimes \pi^{\prime}\right) \neq 0$. By Bernstein's Frobenius reciprocity, this Hom-space is isomorphic to the space of $\left(\widetilde{M}_{t+(n-r)}(F) \times\right.$ $\widetilde{M}_{t}(F)$ )-equivariant maps

$$
\begin{aligned}
& \left(\mu^{\prime}\left|\operatorname{det}_{t+(n-r)}\right|^{\frac{t}{2}} \otimes \mu^{\prime}\left|\operatorname{det}_{t}\right|^{\frac{t+(n-r)}{2}}\right) \otimes C_{c}^{\infty}\left({\widetilde{\operatorname{Sp}_{r-t}}}(F)\right) \\
& \rightarrow R_{\overline{P_{t+(n-r)}(F)}}\left(\left(\mu^{\prime} \circ \operatorname{det}_{n-r}\right) \rtimes \pi\right) \otimes R_{\overline{P_{t}(F)}}\left(\pi^{\prime}\right) .
\end{aligned}
$$

In particular, we have a nonzero $\widetilde{\mathrm{GL}}_{t+(n-r)}(F)$-equivariant map

$$
R_{P_{t+(n-r)}(F)}\left(\left(\mu^{\prime-1} \circ \operatorname{det}_{n-r}\right) \rtimes \pi^{\vee}\right) \rightarrow \mu^{\prime-1}\left|\operatorname{det}_{t+(n-r)}\right|^{-\frac{t}{2}} .
$$

By Proposition A.4, s.s. $R_{P_{t+(n-r)}(F)}\left(\left(\mu^{\prime-1} \circ \operatorname{det}_{n-r}\right) \rtimes \pi^{\vee}\right)$ is the sum of

$$
\text { s.s. }\left(\mu^{\prime}\left|\operatorname{det}_{n-r-a}\right|^{-\frac{a}{2}} \times \mu^{\prime-1}\left|\operatorname{det}_{b}\right|^{-\frac{n-r-b}{2}} \times \tau_{\lambda}\right) \otimes\left(\mu^{\prime-1}\left|\operatorname{det}_{a-b}\right|^{-\frac{n-r-a-b}{2}} \rtimes \pi_{\lambda}\right),
$$

where $(a, b)$ runs over the pairs of integers such that $0 \leq b \leq a \leq n-r$, and $\tau_{\lambda} \otimes \pi_{\lambda}$ runs over all irreducible subquotients of $R_{P_{a-b+t}(F)}\left(\pi^{\vee}\right)$. Hence we have

$$
\text { s.s. }\left(\mu^{\prime}\left|\operatorname{det}_{n-r-a}\right|^{-\frac{a}{2}} \times \mu^{\prime-1}\left|\operatorname{det}_{b}\right|^{-\frac{n-r-b}{2}} \times \tau_{\lambda}\right) \supset \mu^{\prime-1}\left|\operatorname{det}_{t+(n-r)}\right|^{-\frac{t}{2}}
$$

for some $(a, b)$ and $\lambda$. Note that the segments corresponding to $\mu^{\prime}\left|\operatorname{det}_{n-r-a}\right|^{-\frac{a}{2}}, \mu^{\prime-1}\left|\operatorname{det}_{b}\right|^{-\frac{n-r-b}{2}}$ and $\mu^{\prime-1}\left|\operatorname{det}_{t+(n-r)}\right|^{-\frac{t}{2}}$ are $[-(n-r-1) / 2,(n-r-1) / 2-a],[-(n-r-1) / 2,-(n-r-1) / 2+b-1]$ and $[-(n-r-1) / 2-t,(n-r-1) / 2]$, respectively. If $t \geq 1$, then s.s. $R_{P_{1}(F)}\left(\pi^{\vee}\right)$ must contain a 
nonzero representation on which $\widetilde{\mathrm{GL}}_{1}(F)$ acts by $\mu^{\prime-1}|\cdot|^{-(n-r-1) / 2-t}$. This contradicts that $\pi$ is almost tempered, by Casselman's criterion (see e.g., [34, Lemma 2.4]). Hence we must have $t=0$.

Therefore, we obtain a surjection

$$
\operatorname{Ind} \underset{\widetilde{P}_{n-r}(F) \times \widetilde{\operatorname{Sp}}_{r}(F)}{\widetilde{\operatorname{Sp}}_{r}(F)}\left(\left(\mu^{\prime} \circ \operatorname{det}_{n-r}\right) \otimes C_{c}^{\infty}\left(\widetilde{\operatorname{Sp}}_{r}(F)\right)\right) \rightarrow\left(\left(\mu^{\prime} \circ \operatorname{det}_{n-r}\right) \rtimes \pi\right) \otimes \pi^{\prime}
$$

Now Theorem 3.1 (4) follows from the following lemma.

Lemma 3.8. Let $\pi$ be an irreducible almost tempered unitary representation of $\widetilde{\operatorname{Sp}}_{r}(F)$. Then the maximal $\left(\left(\mu^{\prime} \circ \operatorname{det}_{n-r}\right) \rtimes \pi\right)$-isotypic quotient of

$$
\operatorname{Ind}_{\widetilde{P}_{n-r}(F) \times \widetilde{\operatorname{Sp}}_{r}(F)}^{\widetilde{\mathrm{S}}_{n}(F) \widetilde{\mathrm{Sp}}_{r}(F)}\left(\left(\mu^{\prime} \circ \operatorname{det}_{n-r}\right) \otimes C_{c}^{\infty}\left(\widetilde{\operatorname{Sp}}_{r}(F)\right)\right)
$$

is isomorphic to $\left(\left(\mu^{\prime} \circ \operatorname{det}_{n-r}\right) \rtimes \pi\right) \otimes \Pi$, where $\Pi$ is determined so that the subrepresentation of $R_{P_{n-r}(F)}\left(\left(\mu^{\prime-1} \circ \operatorname{det}_{n-r}\right) \rtimes \pi\right)$ on which $\widetilde{\mathrm{GL}}_{n-r}(F)$ acts by $\mu^{\prime-1} \circ \operatorname{det}_{n-r}$ is isomorphic to $\left(\mu^{\prime-1} \circ\right.$ $\left.\operatorname{det}_{n-r}\right) \otimes \Pi$. Moreover, if $\pi$ satisfies one of the conditions in Theorem 3.1 (4), then s.s. $(\Pi)=\pi^{\alpha}$ for some integer $\alpha \geq 1$, and that

$$
\operatorname{dim} \operatorname{Hom}_{\widetilde{\operatorname{Sp}_{r}}(F)}(\Pi, \pi)=1 .
$$

Proof. The linear dual $\Pi^{*}$ of $\Pi$, which is not necessarily smooth, is given by

$$
\Pi^{*} \cong \operatorname{Hom}_{\widetilde{\operatorname{Sp}_{n}}(F)}\left(\operatorname{Ind}_{\widetilde{P_{n-r}}}^{\widetilde{\widetilde{P p}_{n}}(F) \times \widetilde{\widetilde{\operatorname{Sp}}_{r}}(F) \times{\widetilde{\mathrm{Sp}_{r}}}_{r}(F)}\left(\left(\mu^{\prime} \circ \operatorname{det}_{n-r}\right) \otimes C_{c}^{\infty}\left(\widetilde{\operatorname{Sp}_{r}}(F)\right)\right),\left(\mu^{\prime} \circ \operatorname{det}_{n-r}\right) \rtimes \pi\right) .
$$

As representations of $\widetilde{\operatorname{Sp}_{n}}(F)$, we have

$$
\operatorname{Ind} \underset{\widetilde{P}_{n-r}(F) \times \widetilde{\operatorname{Sp}_{r}}(F)}{\widetilde{\operatorname{Sp}}_{r}(F)}\left(\left(\mu^{\prime} \circ \operatorname{det}_{n-r}\right) \otimes C_{c}^{\infty}\left(\widetilde{\operatorname{Sp}_{r}}(F)\right)\right) \cong \operatorname{ind}_{\widetilde{P}_{n-r}^{\circ}(F)}^{\widetilde{S p}_{n}(F)}(\chi)
$$

where

- $P_{n-r}^{\circ}(F) \subset P_{n-r}(F)$ is generated by $\mathrm{GL}_{n-r}(F)$ and $N_{n-r}(F)$, so that $P_{n-r}(F) / P_{n-r}^{\circ}(F) \cong$ $\operatorname{Sp}_{r}(F)$

- ind $\widetilde{P}_{n-r}^{\circ}(F)$ is the (unnormalized) compact induction functor;

- the character $\chi: \widetilde{P}_{n-r}^{\circ}(F) \rightarrow \mathbb{C}^{\times}$is given by

$$
\chi\left(\left(\begin{array}{cc|cc}
a & * & * & * \\
0 & \mathbf{1}_{r} & * & 0 \\
\hline 0 & 0 & { }^{t} a^{-1} & 0 \\
0 & 0 & * & \mathbf{1}_{r}
\end{array}\right), \zeta\right)=\zeta^{n+r}\left(\frac{\alpha_{\psi}(1)}{\alpha_{\psi}(\operatorname{det} a)}\right)^{n+r} \mu(\operatorname{det} a)|\operatorname{det} a|^{\frac{n+r+1}{2}}
$$

Moreover, $\widetilde{\operatorname{Sp}_{r}}(F)$ acts on $\operatorname{ind}_{\widetilde{P}_{n-r}^{\circ}(F)}^{\widetilde{S p}_{n}(F)}(\chi)$ by

$$
\left(g_{r} \cdot \varphi\right)\left(g_{n}\right)=\varphi\left(\left(g_{r}^{-1}\right)^{\alpha} g_{n}\right)
$$

for $g_{r} \in \widetilde{\operatorname{Sp}_{r}}(F), g_{n} \in{\widetilde{\operatorname{Sp}_{n}}}_{n}(F)$ and $\varphi \in \operatorname{ind}_{\widetilde{P}_{n-r}^{\circ}(F)}^{\widetilde{S p}_{n}(F)}(\chi)$. Here, we set

$$
g_{r}^{\alpha}=\left(\begin{array}{cc}
\mathbf{1}_{r} & 0 \\
0 & -\mathbf{1}_{r}
\end{array}\right) g_{r}\left(\begin{array}{cc}
\mathbf{1}_{r} & 0 \\
0 & -\mathbf{1}_{r}
\end{array}\right)^{-1}
$$


and we identify $\widetilde{\mathrm{Sp}_{r}}(F)$ with the image of the composition $\widetilde{\mathrm{Sp}_{r}}(F) \hookrightarrow \widetilde{M}_{n-r}(F) \hookrightarrow \widetilde{\mathrm{Sp}_{n}}(F)$. By the Frobenius reciprocity, we have

$$
\begin{aligned}
\Pi^{*} & \cong \operatorname{Hom}_{\widetilde{\mathrm{Sp}}_{n}(F)}\left(\left(\mu^{\prime-1} \circ \operatorname{det}_{n-r}\right) \rtimes \pi^{\vee}, \operatorname{Ind}_{\widetilde{P}_{n-r}^{\circ}(F)}^{\widetilde{\mathrm{Sp}}_{n}(F)}\left(\chi^{-1} \delta_{P_{n-r}}\right)\right) \\
& \cong \operatorname{Hom}_{\widetilde{P}_{n-r}^{\circ}(F)}\left(\left.\left(\mu^{\prime-1} \circ \operatorname{det}_{n-r}\right) \rtimes \pi^{\vee}\right|_{\widetilde{P}_{n-r}^{\circ}(F)}, \chi^{-1} \delta_{P_{n-r}}\right) \\
& \cong \operatorname{Hom}_{\widetilde{\mathrm{GL}}_{n-r}(F)}\left(\left.R_{P_{n-r}(F)}\left(\left(\mu^{\prime-1} \circ \operatorname{det}_{n-r}\right) \rtimes \pi^{\vee}\right)\right|_{\widetilde{\mathrm{GL}}_{n-r}(F)}, \mu^{\prime-1} \circ \operatorname{det}_{n-r}\right) .
\end{aligned}
$$

Moreover, $\widetilde{\operatorname{Sp}}_{r}(F)$ acts on this Hom-space by

$$
\left(g_{r} \cdot \Phi\right)(f)=\Phi\left(\left(g_{r}^{-1}\right)^{\alpha} \cdot f\right)
$$

for $\Phi$ in this Hom-space, and for $f \in R_{P_{n-r}(F)}\left(\left(\mu^{\prime-1} \circ \operatorname{det}_{n-r}\right) \rtimes \pi^{\vee}\right)$. Since the representation $\left[g_{r} \mapsto \pi^{\vee}\left(g_{r}^{\alpha}\right)\right]$ is isomorphic to $\pi$, by the definition of the Jacquet module, we see that the action $f \mapsto g_{r}^{\alpha} \cdot f$ is isomorphic to $R_{P_{n-r}(F)}\left(\left(\mu^{\prime-1} \circ \operatorname{det}_{n-r}\right) \rtimes \pi\right)$. Hence

$$
\Pi^{*} \cong \operatorname{Hom}_{\widetilde{\mathrm{GL}}_{n-r}(F)}\left(\left.R_{P_{n-r}(F)}\left(\left(\mu^{\prime-1} \circ \operatorname{det}_{n-r}\right) \rtimes \pi\right)\right|_{\widetilde{\mathrm{GL}}_{n-r}(F)}, \mu^{\prime-1} \circ \operatorname{det}_{n-r}\right) .
$$

as representations of $\widetilde{\mathrm{Sp}}_{r}(F)$. This means that $\Pi$ is determined so that the subrepresentation of $R_{P_{n-r}(F)}\left(\left(\mu^{\prime-1} \circ \operatorname{det}_{n-r}\right) \rtimes \pi\right)$ on which $\widetilde{G L}_{n-r}(F)$ acts by $\mu^{\prime-1} \circ \operatorname{det}_{n-r}$ is isomorphic to $\left(\mu^{\prime-1} \circ\right.$ $\left.\operatorname{det}_{n-r}\right) \otimes \Pi$ since $R_{P_{n-r}(F)}\left(\left(\mu^{\prime-1} \circ \operatorname{det}_{n-r}\right) \rtimes \pi\right)$ is admissible.

Note that s.s. $R_{P_{n-r}(F)}\left(\left(\mu^{\prime-1} \circ \operatorname{det}_{n-r}\right) \rtimes \pi\right)$ is the sum of

$$
I_{(a, b), \lambda}=\text { s.s. }\left(\mu^{\prime}\left|\operatorname{det}_{n-r-a}\right|^{-\frac{a}{2}} \times \mu^{\prime-1}\left|\operatorname{det}_{b}\right|^{-\frac{n-r-b}{2}} \times \tau_{\lambda}\right) \otimes\left(\mu^{\prime-1}\left|\operatorname{det}_{a-b}\right|^{-\frac{n-r-a-b}{2}} \rtimes \pi_{\lambda}\right),
$$

where $(a, b)$ runs over the pairs of integers such that $0 \leq b \leq a \leq n-r$, and $\tau_{\lambda} \otimes \pi_{\lambda}$ runs over all irreducible subquotients of $R_{P_{a-b}(F)}(\pi)$. We claim that when $\pi$ satisfies (a) or (b) in Theorem 3.1 (4), if

$$
\left.I_{(a, b), \lambda}\right|_{\widetilde{\mathrm{GL}}_{n-r}(F)} \supset \mu^{\prime-1} \circ \operatorname{det}_{n-r},
$$

then $I_{(a, b), \lambda} \cong \mu^{\prime-1} \circ \operatorname{det}_{n-r} \otimes \pi$.

Note that the segments corresponding to $\mu^{\prime}\left|\operatorname{det}_{n-r-a}\right|^{-\frac{a}{2}}, \mu^{\prime-1}\left|\operatorname{det}_{b}\right|^{-\frac{n-r-b}{2}}$ and $\mu^{\prime-1} \circ \operatorname{det}_{n-r}$ are $[-(n-r-1) / 2,(n-r-1) / 2-a],[-(n-r-1) / 2,-(n-r-1) / 2+b-1]$ and $[-(n-r-1) / 2,(n-r-1) / 2]$, respectively. When the $L$-parameter $\phi$ does not contain $\mu^{ \pm 1} S_{d}$ for any $d \geq n-r$ with $d \equiv n-r$ mod 2 , by computing the Jacquet module with respect to the Borel subgroup of $\widetilde{\mathrm{GL}}_{n-r}(F)$, we see that if

$$
\text { s.s. }\left(\mu^{\prime}\left|\operatorname{det}_{n-r-a}\right|^{-\frac{a}{2}} \times \mu^{\prime-1}\left|\operatorname{det}_{b}\right|^{-\frac{n-r-b}{2}} \times \tau_{\lambda}\right) \supset \mu^{\prime-1} \circ \operatorname{det}_{n-r},
$$

then $(a, b)=(0,0)$ or $(a, b)=(n-r, n-r)$. In these cases, we have $I_{(a, b), \lambda}=\mu^{\prime \pm 1} \circ \operatorname{det}_{n-r} \otimes \pi$, as desired.

When $n=r$, there is nothing to prove. Now we assume that $n=r+1$. We take the maximal integer $a$ such that

$$
\pi \cong \underbrace{\mu^{\prime-1} \times \cdots \times \mu^{\prime-1}}_{a-1} \rtimes \pi_{0}
$$

for some irreducible representation $\pi_{0}$. Then by induction on $a$, one can show that the subrepresentation of s.s. $R_{P_{1}(F)}\left(\mu^{\prime-1} \rtimes \pi\right)$ on which $\widetilde{G L}_{1}(F)$ acts by $\mu^{\prime \pm 1}$ is isomorphic to

$$
\left(\left(\mu^{\prime} \otimes \pi\right) \oplus\left(\mu^{\prime-1} \otimes \pi\right)\right)^{\oplus a} .
$$

This proves the claim when $n=r+1$. 
When $\pi$ satisfies one of the conditions in Theorem 3.1 (4), by the claim, we see that s.s.( $\Pi)$ is of the form $\pi^{\oplus \alpha}$ for some integer $\alpha \geq 1$. Moreover, we have

$$
\begin{aligned}
& \operatorname{Hom}_{\widetilde{\operatorname{Sp}_{r}}(F)}(\Pi, \pi) \cong\left(\Pi^{*} \otimes \pi\right)^{\widetilde{\operatorname{Sp}}_{r}(F)} \\
& \cong \operatorname{Hom}_{\widetilde{\mathrm{GL}_{n-r}}}(F) \\
& \cong \operatorname{End}_{\widetilde{\mathrm{Sp}_{r}}(F)}\left(\left(\mu^{\prime-1} \circ \operatorname{det}_{n-r}\right) \rtimes \pi\right),
\end{aligned}
$$

which is one dimensional by Theorem 3.1 (2).

Remark 3.9. (1) If the L-parameter $\phi$ contains one of $\mu S_{d}$ or $\mu^{-1} S_{d}$ for some $d \geq n-r$ with $d \equiv n-r \bmod 2$, then $\phi \supset \mu S_{d} \oplus \mu^{-1} S_{d}$. By comparing the dimensions, we have

$$
2 r+1-\delta \geq 2 d \geq 2(n-r) .
$$

Hence we must have $n \leq 2 r$. In other words, when $n>2 r$, the condition (a) in Theorem 3.1 (4) always holds.

(2) In general, II in Lemma 3.8 might have irreducible subquotients other than $\pi$. For example, consider the case where $r=2, n=4$ and $\pi=\mu^{\prime-1} \mathrm{St}_{2} \rtimes \mathbf{1}_{\mathrm{Sp}_{0}(F)}$. Then one can see that

$$
\text { s.s. }(\Pi) \supset\left(\mu^{\prime-1} \circ \operatorname{det}_{2}\right) \rtimes \mathbf{1}_{\operatorname{Sp}_{0}(F)} \nsucc \pi \text {. }
$$

3.8. Seesaw identities. In the theory of theta liftings, seesaw identities are useful tools. The following proposition is an analogy for Miyawaki liftings. Recall that $J_{n-1}(F)=\operatorname{Sp}_{n-1}(F) \ltimes V_{n-1}(F)$ is a Jacobi subgroup of $\operatorname{Sp}_{n}(F)$. The center of the Heisenberg group $V_{n-1}(F)$ is denoted by $Z_{n-1}(F)$. For $\xi \in F^{\times}$, we denote the Weil representations of $\widetilde{J}_{n-1}(F)$ and $\widetilde{\operatorname{Sp}}_{r}(F)$ with respect to $\psi_{\xi}$ by $\omega_{\psi_{\xi}}^{(n-1)}$ and $\omega_{\psi_{\xi}}^{(r)}$, respectively.

Proposition 3.10 (Seesaw identity). Let $\pi$ and $\pi^{\prime}$ be irreducible representations of $\widetilde{\mathrm{Sp}}_{r}(F)$ and $\widetilde{\mathrm{Sp}}_{n-1}(F)$, on which $\{ \pm 1\}$ acts by $( \pm 1)^{r+n}$ and $( \pm 1)^{r+n-1}$, respectively. Then

$$
\operatorname{Hom}_{\widetilde{J}_{n-1}(F)}\left(\left.\mathcal{M}_{\psi, \tau}^{(n)}(\pi)\right|_{\widetilde{J}_{n-1}(F)}, \pi^{\prime} \otimes \omega_{\psi_{\xi}}^{(n-1)}\right) \cong \operatorname{Hom}_{\widetilde{\operatorname{Sp}}_{r}(F)}\left(\mathcal{M}_{\psi, \tau \chi_{\xi}}^{(r)}\left(\pi^{\prime}\right) \otimes \omega_{\psi_{\xi}}^{(r)}, \pi\right) .
$$

We shall write this property as the following seesaw diagram:

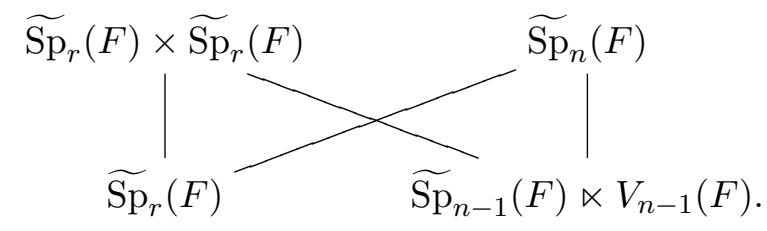

Proof. By proposition 2.2, there exists a $\widetilde{J}_{n+r-1}(F)$-surjection

$$
I_{\psi}^{(n+r)}(\tau) \rightarrow I_{\psi}^{(n+r-1)}\left(\tau \chi_{\xi}\right) \otimes \omega_{\psi_{\xi}}^{(n+r-1)} .
$$

Note that as $\widetilde{J}_{n-1}(F) \times \widetilde{\operatorname{Sp}}_{r}(F)$-modules, we have $\omega_{\psi_{\xi}}^{(n+r-1)} \cong \omega_{\psi_{\xi}}^{(n-1)} \otimes \omega_{\psi_{\xi}}^{(r)}$. Composing a surjective $\widetilde{\operatorname{Sp}}_{n-1}(F) \times \widetilde{\operatorname{Sp}}_{r}(F)-$ map

$$
I_{\psi}^{(n+r-1)}\left(\tau \chi_{\xi}\right) \rightarrow \pi^{\prime} \otimes \mathcal{M}_{\psi, \tau \chi_{\xi}}^{(r)}\left(\pi^{\prime}\right),
$$

we obtain a nonzero $\widetilde{J}_{n-1}(F) \times \widetilde{\operatorname{Sp}}_{r}(F)$-map

$$
I_{\psi}^{(n+r)}(\tau) \rightarrow\left(\pi^{\prime} \otimes \omega_{\psi_{\xi}}^{(n-1)}\right) \otimes\left(\mathcal{M}_{\psi, \tau \chi_{\xi}}^{(r)}\left(\pi^{\prime}\right) \otimes \omega_{\psi_{\xi}}^{(r)}\right)
$$


If $\operatorname{Hom}_{\widetilde{\operatorname{Sp}_{r}}(F)}\left(\mathcal{M}_{\psi, \tau \chi \xi}^{(r)}\left(\pi^{\prime}\right) \otimes \omega_{\psi_{\xi}}^{(r)}, \pi\right) \neq 0$, then we obtain a nonzero $\widetilde{J}_{n-1}(F) \times \widetilde{\operatorname{Sp}}_{r}(F)$-map

$$
I_{\psi}^{(n+r)}(\tau) \rightarrow\left(\pi^{\prime} \otimes \omega_{\psi_{\xi}}^{(n-1)}\right) \otimes \pi .
$$

By the definition of local Miyawaki liftings, it implies a nonzero $\widetilde{J}_{n-1}(F)$-map $\mathcal{M}_{\psi, \tau}^{(n)}(\pi) \rightarrow \pi^{\prime} \otimes \omega_{\psi_{\xi}}^{(n-1)}$ so that

$$
\operatorname{Hom}_{\widetilde{J}_{n-1}(F)}\left(\left.\mathcal{M}_{\psi, \tau}^{(n)}(\pi)\right|_{\widetilde{J}_{n-1}(F)}, \pi^{\prime} \otimes \omega_{\psi_{\xi}}^{(n-1)}\right) \neq 0
$$

Conversely, if $\operatorname{Hom}_{\widetilde{J}_{n-1}(F)}\left(\left.\mathcal{M}_{\psi, \tau}^{(n)}(\pi)\right|_{\widetilde{J}_{n-1}(F)}, \pi^{\prime} \otimes \omega_{\psi_{\xi}}^{(n-1)}\right) \neq 0$, then we have a nonzero $\widetilde{J}_{n-1}(F) \times$ $\widetilde{\mathrm{Sp}}_{r}(F)-\mathrm{map}$

$$
I_{\psi}^{(n+r)}(\tau) \rightarrow \mathcal{M}_{\psi, \tau}^{(n)}(\pi) \otimes \pi \rightarrow\left(\pi^{\prime} \otimes \omega_{\psi_{\xi}}^{(n-1)}\right) \otimes \pi
$$

It factors through

$$
\left(I_{\psi}^{(n+r)}(\tau)\right)_{\psi_{\xi}}
$$

which is the maximal quotient of $I_{\psi}^{(n+r)}(\tau)$ on which $Z_{n+r-1}(F)$ acts by $\psi_{\xi}$. By the proof of Proposition 2.1 together with Proposition 2.2, we see that

$$
\left(I_{\psi}^{(n+r)}(\tau)\right)_{\psi_{\xi}} \cong I_{\psi}^{(n+r-1)}\left(\tau \chi_{\xi}\right) \otimes \omega_{\psi_{\xi}}^{(n+r-1)}
$$

as $\widetilde{J}_{n+r-1}(F)$-modules. Hence we have a nonzero $\widetilde{J}_{n-1}(F) \times{\widetilde{\mathrm{Sp}_{r}}}_{r}(F)$-map

$$
I_{\psi}^{(n+r-1)}\left(\tau \chi_{\xi}\right) \otimes \omega_{\psi_{\xi}}^{(n+r-1)} \rightarrow\left(\pi^{\prime} \otimes \omega_{\psi_{\xi}}^{(n-1)}\right) \otimes \pi
$$

This implies that

$$
\operatorname{Hom}_{\widetilde{\mathrm{Sp}}_{r}(F)}\left(\mathcal{M}_{\psi, \tau \chi_{\xi}}^{(r)}\left(\pi^{\prime}\right) \otimes \omega_{\psi_{\xi}}^{(r)}, \pi\right) \neq 0 .
$$

Hence we obtain two homomorphisms

$$
\operatorname{Hom}_{\widetilde{J}_{n-1}(F)}\left(\left.\mathcal{M}_{\psi, \tau}^{(n)}(\pi)\right|_{\widetilde{J}_{n-1}(F)}, \pi^{\prime} \otimes \omega_{\psi_{\xi}}^{(n-1)}\right) \rightleftarrows \operatorname{Hom}_{\widetilde{\operatorname{Sp}}_{r}(F)}\left(\mathcal{M}_{\psi, \tau \chi_{\xi}}^{(r)}\left(\pi^{\prime}\right) \otimes \omega_{\psi_{\xi}}^{(r)}, \pi\right) .
$$

These are obtained by the following diagram of $\widetilde{J}_{n-1}(F) \times \widetilde{\operatorname{Sp}}_{r}(F)$-homomorphisms

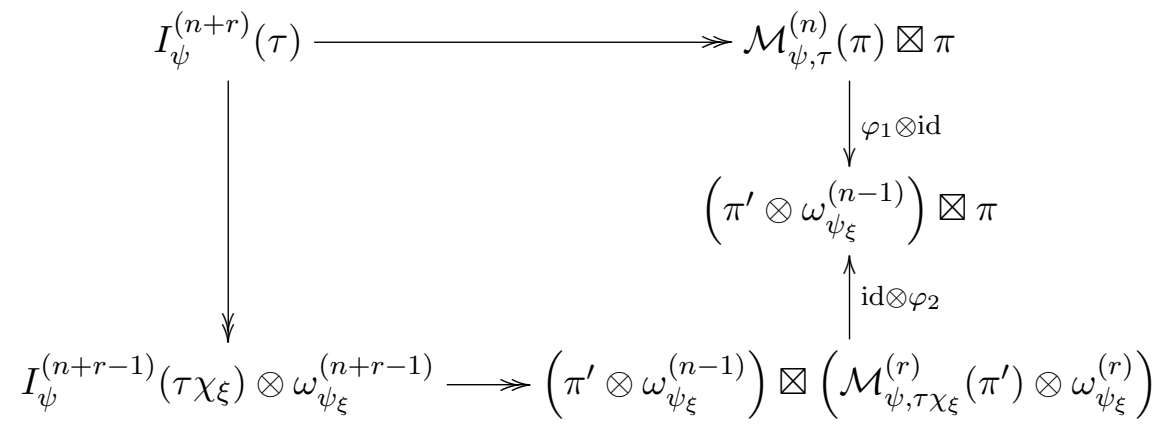

for $\varphi_{1} \in \operatorname{Hom}_{\widetilde{J}_{n-1}(F)}\left(\left.\mathcal{M}_{\psi, \tau}^{(n)}(\pi)\right|_{\widetilde{J}_{n-1}(F)}, \pi^{\prime} \otimes \omega_{\psi_{\xi}}^{(n-1)}\right)$ and $\varphi_{2} \in \operatorname{Hom}_{\widetilde{\operatorname{Sp}}_{r}(F)}\left(\mathcal{M}_{\psi, \tau \chi_{\xi}}^{(r)}\left(\pi^{\prime}\right) \otimes \omega_{\psi_{\xi}}^{(r)}, \pi\right)$. Clearly, the two maps $\varphi_{1} \mapsto \varphi_{2}$ and $\varphi_{2} \mapsto \varphi_{1}$ are inverse to each other. 


\section{Global Miyawaki liftings}

Now we let $F$ be a totally real number field, and $\psi$ be a non-trivial unitary character of $\mathbb{A} / F$. We assume that for each infinite place $v$ of $F$, there exists $a_{v} \in F_{v} \cong \mathbb{R}$ with $a_{v}>0$ such that $\psi\left(x_{v}\right)=\exp \left(2 \pi a_{v} \sqrt{-1} x_{v}\right)$ for $x_{v} \in F_{v}$.

In this section, we define the global Miyawaki liftings as the pullbacks of Ikeda liftings, and establish basic properties. One may regard the global Miyawaki liftings as an analogue of the global theta liftings, which are defined by the pullbacks of theta functions.

4.1. Definition. Let $\tau=\otimes_{v}^{\prime} \tau_{v}$ be an irreducible unitary cuspidal automorphic representation of $\mathrm{GL}_{2}(\mathbb{A})$ satisfying the conditions (A1), (A2) and (A3) in $\S 2.5$. We denote the weight of $\otimes_{v \mid \infty} \tau_{v}$ by $\pm 2 k=\left( \pm 2 k_{v}\right)_{v}$ with $k_{v}>0$. Then we have the Ikeda lift $\operatorname{Ik}_{\psi}^{(n+r)}(\tau)$, which is an irreducible

admissible representation of $\widetilde{\operatorname{Sp}}_{n+r}\left(\mathbb{A}_{\text {fin }}\right)$ occurring in the space $\mathcal{S}_{k+(n+r) / 2}\left(\operatorname{Sp}_{n+r}(F) \backslash \widetilde{\operatorname{Sp}}_{n+r}(\mathbb{A})\right)$ of holomorphic cusp forms on $\widetilde{\mathrm{Sp}}_{n+r}(\mathbb{A})$ of weight $k+(n+r) / 2$. For $\mathcal{F} \in \operatorname{Ik}_{\psi}^{(n+r)}(\tau)$ and $\varphi \in$ $\mathcal{S}_{k+(n+r) / 2}\left(\operatorname{Sp}_{r}(F) \backslash \widetilde{\operatorname{Sp}}_{r}(\mathbb{A})\right)$, consider the integral

$$
\mathcal{M}^{(n)}\left(\left(g_{n}, \zeta_{n}\right) ; \varphi, \mathcal{F}\right)=\int_{\operatorname{Sp}_{r}(F) \backslash \operatorname{Sp}_{r}(\mathbb{A})} \mathcal{F}\left(\iota\left(g_{n}, g_{r}\right), \zeta_{n} \zeta_{r}\right) \overline{\varphi\left(g_{r}, \zeta_{r}\right)} d g_{r}
$$

for $\left(g_{n}, \zeta_{n}\right) \in \widetilde{\operatorname{Sp}_{n}}(\mathbb{A})$, where $d g_{r}$ is the Tamagawa measure on $\operatorname{Sp}_{r}(F) \backslash \operatorname{Sp}_{r}(\mathbb{A})$. Note that this integral does not depend on the choice of $\zeta_{r} \in\{ \pm 1\}$, and that $\mathcal{M}^{(n)}(\varphi, \mathcal{F})$ is genuine if and only if $n+r$ is odd.

Lemma 4.1. We have

$$
\mathcal{M}^{(n)}(\varphi, \mathcal{F}) \in \mathcal{S}_{k+(n+r) / 2}\left(\operatorname{Sp}_{n}(F) \backslash \widetilde{\operatorname{Sp}_{n}}(\mathbb{A})\right)
$$

Proof. The non-trivial part is the cuspidality. Note that $\mathcal{M}^{(n)}(\varphi, \mathcal{F})$ has a Fourier expansion of the form

$$
\mathcal{M}^{(n)}\left(\left(g_{n}, \zeta_{n}\right) ; \varphi, \mathcal{F}\right)=\sum_{B \in \operatorname{Sym}_{n}^{+}(F)} W_{B}\left(g_{n}, \zeta_{n}\right)
$$

where

$$
W_{B}\left(g_{n}, \zeta_{n}\right)=\int_{\operatorname{Sym}_{n}(F) \backslash \operatorname{Sym}_{n}(\mathbb{A})} \mathcal{M}^{(n)}\left(\mathbf{n}(X)\left(g_{n}, \zeta_{n}\right) ; \varphi, \mathcal{F}\right) \overline{\psi(\operatorname{Tr}(B X))} d X .
$$

Since

$$
\mathbf{v}(0, y, z) \mapsto \psi\left(\operatorname{Tr}\left(B\left(\begin{array}{cc}
z & y \\
t^{y} & 0
\end{array}\right)\right)\right)
$$

is a non-trivial character of $Y(\mathbb{A}) Z(\mathbb{A})$ for any $B \in \operatorname{Sym}_{n}^{+}(F)$, we have

$$
\int_{Y(F) Z(F) \backslash Y(\mathbb{A}) Z(\mathbb{A})} \mathcal{M}^{(n)}\left(\mathbf{v}(0, y, z)\left(g_{n}, \zeta_{n}\right) ; \varphi, \mathcal{F}\right) d y d z=0 .
$$

Since $Y Z$ is a normal subgroup of $N_{k}$, which is the unipotent radical of the standard maximal parabolic subgroup $P_{k}$, the constant term of $\mathcal{M}^{(n)}(\varphi, \mathcal{F})$ along $P_{k}$ must be zero. Hence $\mathcal{M}^{(n)}(\varphi, \mathcal{F})$ is cuspidal. 
Let $\pi$ be an admissible (not necessarily irreducible) representation of $\widetilde{\mathrm{Sp}_{r}}\left(\mathbb{A}_{\text {fin }}\right)$ occurring in $\mathcal{S}_{k+(n+r) / 2}\left(\operatorname{Sp}_{r}(F) \backslash \widetilde{\operatorname{Sp}_{r}}(\mathbb{A})\right)$. The (global) Miyawaki lift $\mathcal{M}_{\psi, \tau}^{(n)}(\pi)$ of $\pi$ is defined by the representation of $\widetilde{\mathrm{Sp}_{n}}\left(\mathbb{A}_{\text {fin }}\right)$ generated by

$$
\left\{\mathcal{M}^{(n)}(\varphi, \mathcal{F}) \in \mathcal{S}_{k+(n+r) / 2}\left(\operatorname{Sp}_{n}(F) \backslash \widetilde{\operatorname{Sp}}_{n}(\mathbb{A})\right) \mid \mathcal{F} \in \operatorname{Ik}_{\psi}^{(n+r)}(\tau), \varphi \in \pi\right\} .
$$

4.2. A-parameters. Arthur's multiplicity formula established by Arthur [3] and Gan-Ichino [14] describes the discrete spectrum of automorphic forms on $\operatorname{Sp}_{n}(F) \backslash \widetilde{\operatorname{Sp}}_{n}(\mathbb{A})$ in terms of global $A$ parameters. In this subsection, we review Arthur's multiplicity formula for holomorphic cusp forms. For more precision, see also Appendix B.4.

A discrete global $A$-parameter for $\operatorname{Sp}_{n}(F)\left(\right.$ resp. $\left.\widetilde{\operatorname{Sp}_{n}}(F)\right)$ is a symbol

$$
\Psi=\tau_{1}\left[d_{1}\right] \boxplus \cdots \boxplus \tau_{t}\left[d_{t}\right],
$$

where $\tau_{i}$ is an irreducible cuspidal unitary automorphic representation of $\mathrm{GL}_{m_{i}}(\mathbb{A})$, and $d_{i}$ is a positive integer satisfying several conditions, e.g., $\sum_{i=1}^{t} m_{i} d_{i}$ is equal to $2 n+1$ (resp. $2 n$ ). The precise definition is given in Appendix B.4. Two $A$-parameters $\Psi=\boxplus_{i=1}^{t} \tau_{i}\left[d_{i}\right]$ and $\Psi^{\prime}=\boxplus_{i=1}^{t^{\prime}} \tau_{i}^{\prime}\left[d_{i}^{\prime}\right]$ are said to be equivalent if $t=t^{\prime}$ and there exists a permutation $\sigma \in \mathfrak{S}_{t}$ such that $d_{i}^{\prime}=d_{\sigma(i)}$ and $\tau_{i}^{\prime} \cong \tau_{\sigma(i)}$ for each $i$. We denote the set of equivalence classes of discrete global $A$-parameters for $\operatorname{Sp}_{n}(F)\left(\operatorname{resp} . \widetilde{\operatorname{Sp}}_{n}(F)\right)$ by $\Psi_{2}\left(\operatorname{Sp}_{n}(F)\right)\left(\right.$ resp. $\left.\Psi_{2}\left(\widetilde{\operatorname{Sp}}_{n}(F)\right)\right)$. We call an $A$-parameter $\Psi=\boxplus_{i=1}^{t} \tau_{i}\left[d_{i}\right]$ tempered if $d_{i}=1$ for any $i$. In this case, we write $\Psi=\boxplus_{i=1}^{t} \tau_{i}$ for simplicity.

We state Arthur's multiplicity formula ([3, Theorem 1.5.2] and [14, Theorems 1.1, 1.3]) for holomorphic cusp forms.

Theorem 4.2 (Arthur's multiplicity formula). Let $l=\left(l_{v}\right) \in \prod_{v \mid \infty} \mathbb{Z}$ with $l_{v}>0$ and $l_{v} \equiv l_{v^{\prime}} \bmod 2$ for any $v, v^{\prime} \mid \infty$.

(1) For $\Psi \in \Psi_{2}\left(\operatorname{Sp}_{n}(F)\right)$ if $l_{v}$ is even, and for $\Psi \in \Psi_{2}\left(\widetilde{\operatorname{Sp}_{n}}(F)\right)$ if $l_{v}$ is odd, there exists an $\widetilde{\operatorname{Sp}}_{n}\left(\mathbb{A}_{\text {fin }}\right)$-stable subspace $\mathcal{S}_{l / 2, \Psi}$ of $\mathcal{S}_{l / 2}\left(\operatorname{Sp}_{n}(F) \backslash \widetilde{\operatorname{Sp}}_{n}(\mathbb{A})\right)$ (possibly zero) such that

$$
\mathcal{S}_{l / 2}\left(\operatorname{Sp}_{n}(F) \backslash \widetilde{\operatorname{Sp}}_{n}(\mathbb{A})\right)=\bigoplus_{\Psi} \mathcal{S}_{l / 2, \Psi}
$$

where $\Psi$ runs over $\Psi_{2}\left(\operatorname{Sp}_{n}(F)\right)$ if $l_{v}$ is even, and over $\Psi_{2}\left(\widetilde{\operatorname{Sp}}_{n}(F)\right)$ if $l_{v}$ is odd.

(2) Suppose that $\pi=\otimes_{v<\infty}^{\prime} \pi_{v}$ is an irreducible subrepresentation of $\mathcal{S}_{l / 2, \Psi}$ with $\Psi=\boxplus_{i=1}^{t} \tau_{i}\left[d_{i}\right]$. Then for almost all $v<\infty$, the local factors $\pi_{v}$ of $\pi$ and $\tau_{i, v}$ of $\tau_{i}$ are unramified for any $i$. Moreover, if we denote the Satake parameter for $\tau_{i, v}$ by $\left\{c_{i, v, 1}, \ldots, c_{i, v, m_{i}}\right\}$, then the Satake parameter of $\pi_{v}$ is equal to

$$
\bigcup_{i=1}^{t} \bigcup_{j=1}^{m_{i}}\left\{c_{i, v, j} q^{-\frac{d_{i}-1}{2}}, c_{i, v, j} q^{-\frac{d_{i}-3}{2}}, \ldots c_{i, v, j} q^{\frac{d_{i}-1}{2}}\right\}
$$

as multisets.

(3) If $\Psi$ is a tempered A-parameter, then $\mathcal{S}_{l / 2, \Psi}$ is multiplicity-free as a representation of $\widetilde{\operatorname{Sp}}_{n}\left(\mathbb{A}_{\mathrm{fin}}\right)$.

If an admissible representation $\pi$ of $\widetilde{\operatorname{Sp}_{n}}\left(\mathbb{A}_{\text {fin }}\right)$ is contained in $\mathcal{S}_{l / 2, \Psi}$, we say that $\pi$ has an $A$ parameter $\Psi$, and $\Psi$ is the $A$-parameter for $\pi$. 
4.3. Basic properties. We establish basic properties of Miyawaki liftings. Let $\pi=\otimes_{v<\infty}^{\prime} \pi_{v}$ be an irreducible admissible representation of $\widetilde{\operatorname{Sp}_{r}}\left(\mathbb{A}_{\text {fin }}\right)$ occurring in $\mathcal{S}_{k+(n+r) / 2}\left(\operatorname{Sp}_{r}(F) \backslash \widetilde{\operatorname{Sp}_{r}}(\mathbb{A})\right)$.

First we compute the $A$-parameter for Miyawaki liftings.

Proposition 4.3. Suppose that $\mathcal{M}_{\psi, \tau}^{(n)}(\pi) \neq 0$ with $n \geq r$. If $\pi$ has an A-parameter $\Psi$, then $\mathcal{M}_{\psi, \tau}^{(n)}(\pi)$ has an A-parameter

$$
\Psi \boxplus \tau \chi_{-1}^{[(n+r) / 2]}[n-r] .
$$

Here, when $n=r$, we omit $\tau \chi_{-1}^{[(n+r) / 2]}[n-r]$ so that $\mathcal{M}_{\psi, \tau}^{(r)}(\pi)$ has the same A-parameter as $\pi$.

Proof. This follows from the computation of Satake parameters for $\mathcal{M}_{\psi_{v}, \tau_{v}}^{(n)}\left(\pi_{v}\right)$ (Theorem $\left.3.1(5)\right)$.

Miyawaki liftings have the following duality property.

Proposition 4.4. Suppose that $\mathcal{M}_{\psi, \tau}^{(n)}(\pi) \neq 0$. Then

$$
\pi \subset \mathcal{M}_{\psi, \tau}^{(r)}\left(\mathcal{M}_{\psi, \tau}^{(n)}(\pi)\right)
$$

Proof. For $\varphi_{1}, \varphi_{2} \in \mathcal{S}_{k+(n+r) / 2}\left(\operatorname{Sp}_{r}(F) \backslash \widetilde{\operatorname{Sp}}_{r}(\mathbb{A})\right)$, we define the Petersson inner product by

$$
\left\langle\varphi_{1}, \varphi_{2}\right\rangle=\int_{\operatorname{Sp}_{r}(F) \backslash \mathrm{Sp}_{r}(\mathbb{A})} \varphi_{1}(g, \zeta) \overline{\varphi_{2}(g, \zeta)} d g
$$

If $\mathcal{M}^{(n)}(\varphi, \mathcal{F}) \neq 0$ for $\varphi \in \pi$ and $\mathcal{F} \in \operatorname{Ik}_{\psi}^{(n+r)}(\tau)$, then

$$
\begin{aligned}
& \left\langle\mathcal{M}^{(r)}\left(\mathcal{M}^{(n)}(\varphi, \mathcal{F}), \mathcal{F}\right), \varphi\right\rangle \\
& =\int_{\operatorname{Sp}_{r}(F) \backslash \operatorname{Sp}_{r}(\mathbb{A})}\left(\int_{\operatorname{Sp}_{n}(F) \backslash \operatorname{Sp}_{n}(\mathbb{A})} \mathcal{F}\left(\iota\left(g_{n}, g_{r}\right), \zeta_{n} \zeta_{r}\right) \overline{\mathcal{M}^{(n)}\left(\left(g_{n}, \zeta_{n}\right) \varphi, \mathcal{F}\right)} d g_{n}\right) \overline{\varphi\left(g_{r}, \zeta_{r}\right)} d g_{r} \\
& =\left\langle\mathcal{M}^{(n)}(\varphi, \mathcal{F}), \mathcal{M}^{(n)}(\varphi, \mathcal{F})\right\rangle \neq 0 .
\end{aligned}
$$

Hence $\langle$,$\rangle is nonzero on \mathcal{M}_{\psi, \tau}^{(r)}\left(\mathcal{M}_{\psi, \tau}^{(n)}(\pi)\right) \times \pi$. This shows that $\pi \subset \mathcal{M}_{\psi, \tau}^{(r)}\left(\mathcal{M}_{\psi, \tau}^{(n)}(\pi)\right)$.

The local irreducibility (Theorem 3.1) implies the global irreducibility.

Theorem 4.5. Suppose that $\pi$ has a tempered A-parameter, and $\mathcal{M}_{\psi, \tau}^{(n)}(\pi) \neq 0$ with $n \geq r$. Then:

(1) $\mathcal{M}_{\psi, \tau}^{(n)}(\pi)$ is irreducible, and $\mathcal{M}_{\psi, \tau}^{(n)}(\pi) \cong \otimes_{v<\infty}^{\prime} \mathcal{M}_{\psi_{v}, \tau_{v}}^{(n)}\left(\pi_{v}\right)$.

(2) When $n \leq r+1$ or $n>2 r$, we have

$$
\mathcal{M}_{\psi, \tau}^{(r)}\left(\mathcal{M}_{\psi, \tau}^{(n)}(\pi)\right)=\pi
$$

Proof. Since $\pi$ has a tempered $A$-parameter, the local factor $\pi_{v}$ is almost tempered by Lemma B.8. By Theorem $3.1(2)$, the local Miyawaki lift $\mathcal{M}_{\psi_{v}, \tau_{v}}^{(n)}\left(\pi_{v}\right)$ is irreducible. Hence the $\pi$-isotypic quotient of $\operatorname{Ik}_{\psi}^{(n+r)}(\tau)$ is of the form $\pi^{\prime} \otimes \pi$ for some irreducible representation $\pi^{\prime}$ of $\widetilde{\operatorname{Sp}}_{n}\left(\mathbb{A}_{\text {fin }}\right)$. The $\widetilde{\mathrm{Sp}_{r}}\left(\mathbb{A}_{\text {fin }}\right)$-invariant surjection

$$
\mathrm{Ik}_{\psi}^{(n+r)}(\tau) \otimes \bar{\pi} \rightarrow \mathcal{M}_{\psi, \tau}^{(n)}(\pi), \mathcal{F} \otimes \bar{\varphi} \mapsto \mathcal{M}^{(n)}(\varphi, \mathcal{F})
$$


factors through a surjective $\widetilde{\operatorname{Sp}_{n}}\left(\mathbb{A}_{\text {fin }}\right)$-homomorphism $\pi^{\prime} \rightarrow \mathcal{M}_{\psi, \tau}^{(n)}(\pi)$. Since $\pi^{\prime}$ is irreducible, this map must be injective if $\mathcal{M}_{\psi, \tau}^{(n)}(\pi) \neq 0$. Hence $\mathcal{M}_{\psi, \tau}^{(n)}(\pi)$ is irreducible. Moreover, the above $\widetilde{\mathrm{Sp}_{r}}\left(\mathbb{A}_{\text {fin }}\right)$-invariant surjection implies that the local component at $v<\infty$ of $\mathcal{M}_{\psi, \tau}^{(n)}(\pi)$ is isomorphic to $\mathcal{M}_{\psi_{v}, \tau_{v}}^{(n)}\left(\pi_{v}\right)$. Hence we obtain (1).

By Proposition 4.4, we know that

$$
\mathcal{M}_{\psi, \tau}^{(r)}\left(\mathcal{M}_{\psi, \tau}^{(n)}(\pi)\right) \supset \pi
$$

By a similar argument to (1), when $r \leq n \leq r+1$ or $n>2 r$, Theorem 3.1 (4) implies that the left hand side is irreducible. Hence the inclusion is an equality, and we obtain (2).

Corollary 4.6. Suppose that $\pi$ has a tempered A-parameter $\Psi$.

(1) If $n=r$ and $\mathcal{M}_{\psi, \tau}^{(r)}(\pi) \neq 0$, then $\mathcal{M}_{\psi, \tau}^{(r)}(\pi)=\pi$ as a subrepresentation of $\mathcal{S}_{k+r}\left(\operatorname{Sp}_{r}(F) \backslash \operatorname{Sp}_{r}(\mathbb{A})\right)$.

(2) If $n=r+1$ and $\mathcal{M}_{\psi, \tau}^{(r+1)}(\pi) \neq 0$, then $\mathcal{M}_{\psi, \tau}^{(r+1)}(\pi)$ is a unique irreducible subrepresentation of $\mathcal{S}_{k+r+1 / 2}\left(\operatorname{Sp}_{r+1}(F) \backslash \widetilde{\operatorname{Sp}}_{r+1}(\mathbb{A})\right)$ whose local component at $v<\infty$ is isomorphic to $\mu_{v}^{\prime} \rtimes \pi_{v}$ with $\mu_{v}^{\prime}=\mu_{v} \chi_{-1}^{r}$.

Proof. By Theorem 3.1 (3), the local Miyawaki lift $\mathcal{M}_{\psi_{v}, \tau_{v}}^{(n)}\left(\pi_{v}\right)$ is isomorphic to $\pi_{v}$ if $n=r$, and to $\mu_{v}^{\prime} \rtimes \pi_{v}$ if $n=r+1$. By Proposition 4.3, when $n=r$ (resp. $n=r+1$ ), we see that $\mathcal{M}_{\psi, \tau}^{(n)}(\pi)$ has a tempered $A$-parameter $\Psi^{\prime}=\Psi$ (resp. $\Psi^{\prime}=\Psi \boxplus \tau \chi_{-1}^{r}$ ). Since $\mathcal{S}_{k+(n+r) / 2, \Psi^{\prime}}$ is multiplicity-free by Theorem $4.2(3)$, we have $\mathcal{M}_{\psi, \tau}^{(r)}(\pi)=\pi$ if $n=r$, and $\mathcal{M}_{\psi, \tau}^{(r+1)}(\pi)$ satisfies the desired uniqueness property if $n=r+1$.

Let $n^{\prime}=n$ or $n^{\prime}=n-1$. Fix $l, l^{\prime} \in \prod_{v \mid \infty} \mathbb{Z}$ such that $l_{v} \equiv l_{v^{\prime}} \not \equiv l_{v}^{\prime} \equiv l_{v^{\prime}}^{\prime} \bmod 2$ for any $v, v^{\prime} \mid \infty$. For $\varphi \in \mathcal{S}_{l / 2}\left(\operatorname{Sp}_{n}(F) \backslash \widetilde{\operatorname{Sp}_{n}}(\mathbb{A})\right), \varphi^{\prime} \in \mathcal{S}_{l^{\prime} / 2}\left(\operatorname{Sp}_{n^{\prime}}(F) \backslash \widetilde{\operatorname{Sp}}_{n^{\prime}}(\mathbb{A})\right)$, and $\phi \in \mathcal{S}\left(X_{n^{\prime}}(\mathbb{A})\right)$, we define a Fourier-Jacobi period $\mathcal{P}_{n, n^{\prime}, \psi_{\xi}}\left(\varphi, \overline{\varphi^{\prime}}, \phi\right)$ by the integral

$$
\begin{cases}\int_{\operatorname{Sp}_{n}(F) \backslash \operatorname{Sp}_{n}(\mathbb{A})} \varphi(g, \zeta) \overline{\varphi^{\prime}(g, \zeta)} \overline{\Theta_{\psi_{\xi}}^{\phi}(g, \zeta)} d g, & \text { if } n^{\prime}=n, \\ \int_{V_{n-1}(F) \backslash V_{n-1}(\mathbb{A})} \int_{\operatorname{Sp}_{n-1}(F) \backslash \operatorname{Sp}_{n-1}(\mathbb{A})} \varphi(v(g, \zeta)) \overline{\varphi^{\prime}(g, \zeta)} \overline{\Theta_{\psi_{\xi}}^{\phi}(v(g, \zeta))} d g d v, & \text { if } n^{\prime}=n-1 .\end{cases}
$$

Fix a totally positive element $\xi \in F^{\times}$. Recall that $\mathcal{S}(X(\mathbb{A}))_{\xi}$ is the subspace of $\mathcal{S}(X(\mathbb{A}))$ spanned by $\phi=\otimes_{v} \phi_{v}$ such that $\phi_{v}=\phi_{\xi_{v}}^{0}$ for each infinite place $v$. The local seesaw identity (Proposition 3.10 ) is a local analogue of the following result.

Proposition 4.7 (Seesaw identity). Let $\pi$ and $\pi^{\prime}$ be irreducible representations of $\widetilde{\mathrm{Sp}}_{r}\left(\mathbb{A}_{\mathrm{fin}}\right)$ and $\widetilde{\operatorname{Sp}}_{n-1}\left(\mathbb{A}_{\text {fin }}\right)$ occurring in $\mathcal{S}_{k+(n+r) / 2}\left(\operatorname{Sp}_{r}(F) \backslash \widetilde{\operatorname{Sp}_{r}}(\mathbb{A})\right)$ and $\mathcal{S}_{k+(n-1+r) / 2}\left(\operatorname{Sp}_{n-1}(F) \backslash \widetilde{\operatorname{Sp}_{n-1}}(\mathbb{A})\right)$, respectively.

(1) If there exist $\mathcal{M}^{(n)}\left(\varphi, \mathcal{F}_{1}\right) \in \mathcal{M}_{\psi, \tau}^{(n)}(\pi)$ with $\varphi \in \pi, \varphi^{\prime} \in \pi^{\prime}$, and $\phi_{1} \in \mathcal{S}\left(X_{n-1}(\mathbb{A})\right)_{\xi}$ such that

$$
\mathcal{P}_{n, n-1, \psi_{\xi}}\left(\mathcal{M}^{(n)}\left(\varphi, \mathcal{F}_{1}\right), \overline{\varphi^{\prime}}, \phi_{1}\right) \neq 0,
$$

then there exist $\mathcal{M}^{(r)}\left(\varphi^{\prime}, \mathcal{F}_{2}^{\prime}\right) \in \mathcal{M}_{\psi, \tau \chi_{\xi}}^{(r)}\left(\pi^{\prime}\right)$ and $\phi_{2} \in \mathcal{S}\left(X_{r}(\mathbb{A})\right)_{\xi}$ such that

$$
\mathcal{P}_{r, r, \psi_{\xi}}\left(\varphi, \overline{\mathcal{M}^{(r)}\left(\varphi^{\prime}, \mathcal{F}_{2}^{\prime}\right)}, \phi_{2}\right) \neq 0
$$


(2) Assume that $n+r \geq 2$. If there exist $\varphi \in \pi, \mathcal{M}^{(r)}\left(\varphi^{\prime}, \mathcal{F}_{2}^{\prime}\right) \in \mathcal{M}_{\psi, \tau \chi_{\xi}}^{(r)}\left(\pi^{\prime}\right)$ with $\varphi^{\prime} \in \pi^{\prime}$, and $\phi_{2} \in \mathcal{S}\left(X_{r}(\mathbb{A})\right)_{\xi}$ such that

$$
\mathcal{P}_{r, r, \psi_{\xi}}\left(\varphi, \overline{\mathcal{M}^{(r)}\left(\varphi^{\prime}, \mathcal{F}_{2}^{\prime}\right)}, \phi_{2}\right) \neq 0
$$

then there exist $\mathcal{M}^{(n)}\left(\varphi, \mathcal{F}_{1}\right) \in \mathcal{M}_{\psi, \tau}^{(n)}(\pi)$ and $\phi_{1} \in \mathcal{S}\left(X_{n-1}(\mathbb{A})\right)_{\xi}$ such that

$$
\mathcal{P}_{n, n-1, \psi_{\xi}}\left(\mathcal{M}^{(n)}\left(\varphi, \mathcal{F}_{1}\right), \overline{\varphi^{\prime}}, \phi_{1}\right) \neq 0 .
$$

Proof. Suppose that there exist $\mathcal{M}^{(n)}(\varphi, \mathcal{F}) \in \mathcal{M}_{\psi, \tau}^{(n)}(\pi)$ with $\varphi \in \pi, \varphi^{\prime} \in \pi^{\prime}$, and $\phi \in \mathcal{S}\left(X_{n-1}(\mathbb{A})\right)_{\xi}$ such that

It is equal to

$$
\mathcal{P}_{n, n-1, \psi_{\xi}}\left(\mathcal{M}^{(n)}(\varphi, \mathcal{F}), \overline{\varphi^{\prime}}, \phi\right) \neq 0
$$

$$
\begin{aligned}
& \left.\int_{V_{n-1}(F) \backslash V_{n-1}(\mathbb{A})} \int_{\operatorname{Sp}_{n-1}(F) \backslash \operatorname{Sp}_{n-1}(\mathbb{A})} \mathcal{F}\left(\iota(v, 1) \cdot \iota\left(g_{n-1}, g_{r}\right), \zeta_{n} \zeta_{r}\right) \overline{\varphi\left(g_{r}, \zeta_{n}\right)} d g_{r}\right) \\
& \quad \times\left(\int_{\operatorname{Sp}_{r}(F) \backslash \operatorname{Sp}_{r}(\mathbb{A})} \overline{\Theta_{\varphi^{\prime}\left(g_{n-1}, \zeta_{n}\right)}} \overline{\Theta_{\psi_{\xi}}^{\phi}\left(v\left(g_{n-1}, \zeta_{n}\right)\right)} d g_{n-1} d v .\right.
\end{aligned}
$$

First, if we compute the integral on $Z_{n-1}(F) \backslash Z_{n-1}(\mathbb{A})$, then $\mathcal{F}_{\psi_{\xi}}$ appears. By Proposition 2.7, there exist $\mathcal{F}_{1}^{\prime}, \ldots, \mathcal{F}_{r}^{\prime} \in \mathrm{Ik}_{\psi}^{(n+r-1)}\left(\tau \otimes \chi_{\xi}\right)$ and $\phi_{1}, \ldots, \phi_{r} \in \mathcal{S}\left(X_{n+r-1}(\mathbb{A})\right)_{\xi}$ such that

$$
\mathcal{F}_{\psi_{\xi}}\left(\iota(v, 1) \cdot \iota\left(g_{n-1}, g_{r}\right), \zeta_{n} \zeta_{r}\right)=\sum_{i=1}^{r} \mathcal{F}_{i}^{\prime}\left(\iota\left(g_{n-1}, g_{r}\right), \zeta_{n} \zeta_{r}\right) \Theta_{\psi_{\xi}}^{\phi_{i}}\left(\iota(v, 1) \cdot \iota\left(g_{n-1}, g_{r}\right), \zeta_{n} \zeta_{r}\right) .
$$

Note that $X_{n+r-1}(\mathbb{A}) \cong X_{n-1}(\mathbb{A}) \oplus X_{r}(\mathbb{A})$. We may assume that $\phi_{i}=\phi_{i}^{(n-1)} \otimes \phi_{i}^{(r)}$ with $\phi_{i}^{(n-1)} \in$ $\mathcal{S}\left(X_{n-1}(\mathbb{A})\right)_{\xi}$ and $\phi_{i}^{(r)} \in \mathcal{S}\left(X_{r}(\mathbb{A})\right)_{\xi}$. Then we have

$$
\Theta_{\psi_{\xi}}^{\phi_{i}}\left(\iota(v, 1) \cdot \iota\left(g_{n-1}, g_{r}\right), \zeta_{n} \zeta_{r}\right)=\Theta_{\psi_{\xi}}^{\phi_{i}^{(n-1)}}\left(v\left(g_{n-1}, \zeta_{n}\right)\right) \Theta_{\psi_{\xi}}^{\phi_{i}^{(r)}}\left(g_{r}, \zeta_{r}\right)
$$

Hence there exists $i$ such that

$$
\begin{aligned}
& \int_{\operatorname{Sp}_{n-1}(F) \backslash \operatorname{Sp}_{n-1}(\mathbb{A})} \int_{\operatorname{Sp}_{r}(F) \backslash \operatorname{Sp}_{r}(\mathbb{A})} \mathcal{F}_{i}^{\prime}\left(\iota\left(g_{n-1}, g_{r}\right), \zeta_{n} \zeta_{r}\right) \overline{\varphi\left(g_{r}, \zeta_{r}\right) \varphi^{\prime}\left(g_{n-1}, \zeta_{n}\right)} \Theta_{\psi_{\xi}}^{\phi_{i}^{(r)}}\left(g_{r}, \zeta_{r}\right) \\
& \times\left(\int_{Z_{n-1}(\mathbb{A}) V_{n-1}(F) \backslash V_{n-1}(\mathbb{A})} \Theta_{\psi_{\xi}^{\phi_{i}^{(n-1)}}}\left(v\left(g_{n-1}, \zeta_{n}\right)\right) \overline{\Theta_{\psi_{\xi}}^{\phi}\left(v\left(g_{n-1}, \zeta_{n}\right)\right)} d v\right) d g_{r} d g_{n-1} \neq 0 .
\end{aligned}
$$

The integral on $Z_{n-1}(\mathbb{A}) V_{n-1}(F) \backslash V_{n-1}(\mathbb{A})$ is equal to the inner product $\left(\phi_{i}^{(n-1)}, \phi\right)$, which does not depend on $\left(g_{n-1}, \zeta_{n}\right)$. The other integral is equal to the complex conjugate of

$$
\mathcal{P}_{r, r, \psi_{\xi}}\left(\varphi, \overline{\mathcal{M}^{(r)}\left(\varphi^{\prime}, \mathcal{F}_{i}^{\prime}\right)}, \phi_{i}^{(r)}\right)
$$

Hence we obtain (1).

Next suppose that there exist $\varphi \in \pi, \mathcal{M}^{(r)}\left(\varphi^{\prime}, \mathcal{F}^{\prime}\right) \in \mathcal{M}_{\psi, \tau \chi \xi}^{(r)}\left(\pi^{\prime}\right)$ with $\varphi^{\prime} \in \pi^{\prime}$, and $\phi_{2} \in \mathcal{S}\left(X_{r}(\mathbb{A})\right)_{\xi}$ such that

$$
\mathcal{P}_{r, r, \psi_{\xi}}\left(\varphi, \overline{\mathcal{M}^{(r)}\left(\varphi^{\prime}, \mathcal{F}^{\prime}\right)}, \phi\right) \neq 0 .
$$


Choose a nonzero vector $\phi^{\prime} \in \mathcal{S}\left(X_{n-1}(\mathbb{A})\right)_{\xi}$. Then by the same calculation above, we have

$$
\begin{aligned}
& \left(\phi^{\prime}, \phi^{\prime}\right) \overline{\mathcal{P}_{r, r, \psi_{\xi}}\left(\varphi, \overline{\mathcal{M}^{(r)}\left(\varphi^{\prime}, \mathcal{F}^{\prime}\right)}, \phi\right)} \\
& =\int_{\operatorname{Sp}_{r}(F) \backslash \operatorname{Sp}_{r}(\mathbb{A})} \int_{\operatorname{Sp}_{n-1}(F) \backslash \operatorname{Sp}_{n-1}(\mathbb{A})} \mathcal{F}^{\prime}\left(\iota\left(g_{n-1}, g_{r}\right), \zeta_{n} \zeta_{r}\right) \overline{\varphi^{\prime}\left(g_{n-1}, \zeta_{n}\right) \varphi\left(g_{r}, \zeta_{r}\right)} \Theta_{\psi_{\xi}}^{\phi}\left(g_{r}, \zeta_{r}\right) \\
& \times\left(\int_{Z_{n-1}(\mathbb{A}) V_{n-1}(F) \backslash V_{n-1}(\mathbb{A})} \Theta_{\psi_{\xi}}^{\phi^{\prime}}\left(v\left(g_{n-1}, \zeta_{n}\right) \overline{\Theta_{\psi_{\xi}}^{\phi^{\prime}}\left(v\left(g_{n-1}, \zeta_{n}\right)\right)} d v\right) d g_{n-1} d g_{r}\right. \\
& =\int_{Z_{n-1}(\mathbb{A}) V_{n-1}(F) \backslash V_{n-1}(\mathbb{A})} \int_{\operatorname{Sp}_{n-1}(F) \backslash \operatorname{Sp}_{n-1}(\mathbb{A})} \\
& \times\left(\int_{\operatorname{Sp}_{r}(F) \backslash \operatorname{Sp}_{r}(\mathbb{A})} \mathcal{F}^{\prime}\left(\iota\left(g_{n-1}, g_{r}\right), \zeta_{n} \zeta_{r}\right) \Theta_{\psi_{\xi}}^{\phi^{\prime} \otimes \phi}\left(\iota(v, 1) \cdot \iota\left(g_{n-1}, g_{r}\right), \zeta_{n} \zeta_{r}\right) \overline{\varphi\left(g_{r}, \zeta_{r}\right)} d g_{r}\right) \\
& \times \overline{\varphi^{\prime}\left(g_{n-1}, \zeta_{n}\right)} \overline{\Theta_{\psi_{\xi}}^{\phi^{\prime}}\left(v\left(g_{n-1}, \zeta_{n}\right)\right)} d g_{n-1} d v \text {. }
\end{aligned}
$$

If $n+r \geq 2$, then by Proposition $2.7(2),(3)$, there exists $\mathcal{F} \in \operatorname{Ik}_{\psi}^{(n+r)}(\tau)$ such that

$$
\mathcal{F}_{\psi_{\xi}}\left(\iota(v, 1) \cdot \iota\left(g_{n-1}, g_{r}\right), \zeta_{n} \zeta_{r}\right)=\mathcal{F}^{\prime}\left(\iota\left(g_{n-1}, g_{r}\right), \zeta_{n} \zeta_{r}\right) \Theta_{\psi_{\xi}}^{\phi^{\prime} \otimes \phi}\left(\iota(v, 1) \cdot \iota\left(g_{n-1}, g_{r}\right), \zeta_{n} \zeta_{r}\right)
$$

Then

$$
0 \neq\left(\phi^{\prime}, \phi^{\prime}\right) \overline{\mathcal{P}_{r, r, \psi_{\xi}}\left(\varphi, \overline{\mathcal{M}^{(r)}\left(\varphi^{\prime}, \mathcal{F}^{\prime}\right)}, \phi\right)}=\mathcal{P}_{n, n-1, \psi_{\xi}}\left(\mathcal{M}^{(n)}(\varphi, \mathcal{F}), \overline{\varphi^{\prime}}, \phi^{\prime}\right)
$$

Hence we obtain (2).

As an application of this seesaw identity, we have a criterion for the non-vanishing of the Miyawaki liftings for the equal rank case.

Proposition 4.8. Let $\pi$ be an irreducible representation of $\operatorname{Sp}_{r}\left(\mathbb{A}_{\text {fin }}\right)$ occurring in $\mathcal{S}_{k+r}\left(\operatorname{Sp}_{r}(F) \backslash \operatorname{Sp}_{r}(\mathbb{A})\right)$ with $r \geq 1$. Assume that $\pi$ has a tempered A-parameter, and that the $\xi$-th Fourier-Jacobi module $\mathrm{FJ}_{\psi_{\xi}}(\pi)$ is nonzero. Then the following are equivalent:

(a) $\mathcal{M}_{\psi, \tau}^{(r)}(\pi)$ is nonzero.

(b) For any irreducible representation $\pi^{\prime}$ of $\widetilde{\mathrm{Sp}}_{r-1}\left(\mathbb{A}_{\mathrm{fin}}\right)$ occurring in $\mathcal{S}_{k+r-1 / 2}\left(\operatorname{Sp}_{r-1}(F) \backslash \widetilde{\mathrm{Sp}}_{r-1}(\mathbb{A})\right)$, if $\mathcal{P}_{r, r-1, \psi_{\xi}}$ is not identically zero on $\pi \times \overline{\pi^{\prime}} \times \mathcal{S}\left(X_{r-1}(\mathbb{A})\right)_{\xi}$, then $\mathcal{P}_{r, r, \psi_{\xi}}$ is not identically zero on $\pi \times \overline{\mathcal{M}_{\psi, \tau \chi_{\xi}}^{(r)}\left(\pi^{\prime}\right)} \times \mathcal{S}\left(X_{r}(\mathbb{A})\right)_{\xi}$.

(c) There exists an irreducible representation $\pi^{\prime}$ of $\widetilde{\operatorname{Sp}}_{r-1}\left(\mathbb{A}_{\text {fin }}\right)$ occurring in $\mathcal{S}_{k+r-1 / 2}\left(\operatorname{Sp}_{r-1}(F) \backslash \widetilde{\operatorname{Sp}}_{r-1}(\mathbb{A})\right)$ such that $\mathcal{P}_{r, r, \psi_{\xi}}$ is not identically zero on $\pi \times \overline{\mathcal{M}_{\psi, \tau \chi_{\xi}}^{(r)}\left(\pi^{\prime}\right)} \times \mathcal{S}\left(X_{r}(\mathbb{A})\right)_{\xi}$.

Proof. First, we show that (a) implies (b). Suppose that $\mathcal{M}_{\psi, \tau}^{(r)}(\pi)$ is nonzero. Since $\pi$ has a tempered $A$-parameter, by Corollary $4.6(1)$, we have $\mathcal{M}_{\psi, \tau}^{(r)}(\pi)=\pi$. Then by Proposition 4.7 (1), we obtain the condition (b).

Next, we show that (b) implies (c). Since $\mathrm{FJ}_{\psi_{\xi}}(\pi) \neq 0$, the map

$$
\begin{aligned}
\pi \otimes \overline{\omega_{\psi_{\xi}}} & \rightarrow \mathcal{S}_{k+r-1 / 2}\left(\operatorname{Sp}_{r-1}(F) \backslash{\widetilde{\operatorname{Sp}_{r-1}}}(\mathbb{A})\right), \\
\varphi \otimes \bar{\phi} & \mapsto \int_{V_{r-1}(F) \backslash V_{r-1}(\mathbb{A})} \varphi\left(v g^{\prime}\right) \cdot \overline{\Theta_{\psi_{\xi}}^{\phi}\left(v g^{\prime}\right)} d v
\end{aligned}
$$


is nonzero. If we take an irreducible subrepresentation $\pi^{\prime}$ in the image of this map, then $\mathcal{P}_{r, r-1, \psi_{\xi}}$ is not identically zero on $\pi \times \overline{\pi^{\prime}} \times \mathcal{S}\left(X_{r-1}(\mathbb{A})\right)_{\xi}$. Applying (b) to $\pi^{\prime}$, we see that $\pi^{\prime}$ satisfies the condition of $(\mathrm{c})$.

Finally, we show that (c) implies (a). If $\mathcal{P}_{r, r, \psi_{\xi}}$ is not identically zero on $\pi \times \overline{\mathcal{M}_{\psi, \tau \chi_{\xi}}^{(r)}\left(\pi^{\prime}\right)} \times$ $\mathcal{S}\left(X_{r}(\mathbb{A})\right)_{\xi}$, then $\mathcal{P}_{r, r-1, \psi_{\xi}}$ is not identically zero on $\mathcal{M}_{\psi, \tau}^{(r)}(\pi) \times \overline{\pi^{\prime}} \times \mathcal{S}\left(X_{r-1}(\mathbb{A})\right)_{\xi}$ by Proposition 4.7 (2). In particular, $\mathcal{M}_{\psi, \tau}^{(r)}(\pi) \neq 0$. This completes the proof.

\section{A relation Between Miyawaki liftings and the Gan-Gross-Prasad conjecture}

In this section, we formulate a conjecture on the non-vanishing of global Miyawaki liftings. In addition, we relate this conjecture to the Gan-Gross-Prasad conjecture for the symplectic-metaplectic case.

5.1. Conjectures. Fix $\tau$ and $\psi$ as in the previous section, and a totally positive element $\xi \in$ $F^{\times}$. We denote the weight of $\otimes_{v \mid \infty} \tau_{v}$ by $\pm 2 k=\left( \pm 2 k_{v}\right)_{v}$ with $k_{v}>0$. Let $\pi$ be an irreducible representation of $\widetilde{\operatorname{Sp}_{r}}\left(\mathbb{A}_{\text {fin }}\right)$ occurring in $\mathcal{S}_{k+(n+r) / 2}\left(\operatorname{Sp}_{r}(F) \backslash \widetilde{\operatorname{Sp}_{r}}(\mathbb{A})\right)$, and $\mathcal{M}_{\psi, \tau}^{(n)}(\pi)$ be the Miyawaki lift of $\pi$. The Rankin-Selberg $L$-function attached to $\pi \times \tau \chi_{-1}^{r}$ is denoted by $L\left(s, \pi \times \tau \chi_{-1}^{r}\right)$.

Conjecture $5.1\left((M)_{r, n}\right)$. Suppose that $n \geq r$.

(1) When $n=r$, the Miyawaki lift $\mathcal{M}_{\psi, \tau}^{(r)}(\pi)$ is nonzero if and only if $L\left(1 / 2, \pi \times \tau \chi_{-1}^{r}\right) \neq 0$.

(2) When $n>r$, the Miyawaki lift $\mathcal{M}_{\psi, \tau}^{(n)}(\pi)$ is always nonzero.

Note that when $r=0$, Conjecture 5.1 is trivial since $\mathcal{M}_{\psi, \tau}^{(n)}\left(\eta^{n}\right)=\operatorname{Ik}_{\psi}^{(n)}(\tau)$, where $\eta$ is the unique non-trivial character of $\widetilde{\operatorname{Sp}_{0}}\left(\mathbb{A}_{\text {fin }}\right) \cong \mathbb{Z} / 2 \mathbb{Z}$. When $n \geq r$ and $n \equiv r \bmod 2$, it is an extension of a part of Ikeda's conjecture [28, Conjecture 5.1]. The simplest case of this conjecture (the case where $F=\mathbb{Q}, n=r=1$, and both $\tau \chi_{-1}$ and $\pi$ are unramified everywhere) was proven by Ichino [25] and Xue [57] independently. When $n \geq r$ and $n \not \equiv r \bmod 2$, some examples were given by Hayashida [22, Lemma 9.1].

In the rest of this section, we explain a relation between Conjecture 5.1 and the Gan-GrossPrasad conjecture.

5.2. Gan-Gross-Prasad conjectures. The Gan-Gross-Prasad conjecture (the GGP conjecture) [11, Conjecture 24.1] relates the non-vanishing of the Fourier-Jacobi periods to the non-vanishing of the central values of the Rankin-Selberg $L$-functions. In this subsection, we review the GGP conjecture for holomorphic cusp forms. For more details, we refer the readers to Appendix C.

Suppose that $n^{\prime}=n$ or $n^{\prime}=n-1$. Let $\pi$ and $\pi^{\prime}$ be irreducible representations of $\widetilde{\operatorname{Sp}}_{n}\left(\mathbb{A}_{\text {fin }}\right)$ and $\widetilde{\operatorname{Sp}}_{n^{\prime}}\left(\mathbb{A}_{\text {fin }}\right)$ occurring in $\mathcal{S}_{l / 2}\left(\operatorname{Sp}_{n}(F) \backslash \widetilde{\operatorname{Sp}_{n}}(\mathbb{A})\right)$ and $\mathcal{S}_{(l-1) / 2}\left(\operatorname{Sp}_{n^{\prime}}(F) \backslash \widetilde{\operatorname{Sp}}_{n^{\prime}}(\mathbb{A})\right)$, respectively. Assume that $\pi$ and $\pi^{\prime}$ have tempered $A$-parameters $\Psi=\boxplus_{i=1}^{t} \tau_{i}$ and $\Psi^{\prime}=\boxplus_{j=1}^{t^{\prime}} \tau_{j}^{\prime}$, respectively. We define the Rankin-Selberg $L$-function $L\left(s, \pi \times \pi^{\prime} \times \chi_{(-1)^{l-1} \xi}\right)$ by

$$
L\left(s, \pi \times \pi^{\prime} \times \chi_{(-1)^{l-1} \xi}\right)=\prod_{i=1}^{t} \prod_{j=1}^{t^{\prime}} L\left(s, \tau_{i} \times \tau_{j}^{\prime} \times \chi_{(-1)^{l-1} \xi}\right) .
$$

Note that $\tau_{j}^{\prime}$ is self-dual and unitary, so that $\overline{\tau_{j}^{\prime}} \cong \tau_{j}^{\prime}$ for any $j$.

Since we consider holomorphic cusp forms only, we state the GGP conjecture as the following unusual way. 
Conjecture 5.2 (Gan-Gross-Prasad conjecture). Let $\pi=\otimes_{v<\infty}^{\prime} \pi_{v}$ and $\pi^{\prime}=\otimes_{v<\infty}^{\prime} \pi_{v}^{\prime}$ be as above.

(1) If the Fourier-Jacobi period $\mathcal{P}_{n, n^{\prime}, \psi_{\xi}}$ is not identically zero on $\pi \times \overline{\pi^{\prime}} \times \mathcal{S}\left(X_{n^{\prime}}(\mathbb{A})\right)_{\xi}$, then the central value $L\left(1 / 2, \pi \times \pi^{\prime} \times \chi_{(-1)^{l-1} \xi}\right)$ is nonzero and the local Hom-space

$$
\operatorname{Hom}_{\mathrm{Sp}_{n^{\prime}}\left(F_{v}\right)}\left(\pi_{v} \otimes \overline{\pi_{v}^{\prime}} \otimes \overline{\omega_{\psi_{\xi}}}, \mathbb{C}\right)
$$

is nonzero for any $v<\infty$.

(2) When $n^{\prime}=n$, the converse of (1) holds.

For the usual statements and the relation with Conjecture 5.2, see Appendix $\mathrm{C}$ below. When $n^{\prime}=n$, Conjecture 5.2 (1) was proven by Yamana [58].

5.3. Relation between Conjectures 5.1 and 5.2. In this subsection, we show Conjecture 5.1 using the GGP conjecture and the following hypothesis.

Hypothesis 5.3. Let $\pi$ be an irreducible representation of $\widetilde{\operatorname{Sp}_{r}}\left(\mathbb{A}_{\text {fin }}\right)$ occurring in $\mathcal{S}_{l / 2}\left(\operatorname{Sp}_{r}(F) \backslash \widetilde{\operatorname{Sp}_{r}}(\mathbb{A})\right)$.

(A) Suppose that $\pi$ has a tempered A-parameter and that $\mathrm{FJ}_{\psi_{\xi}}(\pi) \neq 0$. Then there exists an irreducible representation $\pi^{\prime}$ of $\widetilde{\mathrm{Sp}}_{r-1}\left(\mathbb{A}_{\text {fin }}\right)$ occurring in $\mathcal{S}_{(l-1) / 2}\left(\operatorname{Sp}_{r-1}(F) \backslash \widetilde{\operatorname{Sp}_{r-1}}(\mathbb{A})\right)$ with tempered A-parameter such that $\mathcal{P}_{r, r-1, \psi_{\xi}}$ is not identically zero on $\pi \times \overline{\pi^{\prime}} \times \mathcal{S}\left(X_{r-1}(\mathbb{A})\right)_{\xi}$.

(B) There exists an irreducible representation $\pi^{\prime}$ of ${\widetilde{\operatorname{Sp}_{r}}}_{(}\left(\mathbb{A}_{\text {fin }}\right)$ occurring in $\mathcal{S}_{(l-1) / 2}\left(\operatorname{Sp}_{r}(F) \backslash \widetilde{\operatorname{Sp}}_{r}(\mathbb{A})\right)$ such that $\mathcal{P}_{r, r, \psi_{\xi}}$ is not identically zero on $\pi \times \overline{\pi^{\prime}} \times \mathcal{S}\left(X_{r}(\mathbb{A})\right)_{\xi}$.

(C) There exists an irreducible representation $\pi^{\prime}$ of $\widetilde{\operatorname{Sp}}_{r+1}\left(\mathbb{A}_{\text {fin }}\right)$ occurring in $\mathcal{S}_{(l+1) / 2}\left(\operatorname{Sp}_{r+1}(F) \backslash \widetilde{\operatorname{Sp}}_{r+1}(\mathbb{A})\right)$ such that $\mathcal{P}_{r+1, r, \psi_{\xi}}$ is not identically zero on $\pi^{\prime} \times \bar{\pi} \times \mathcal{S}\left(X_{r}(\mathbb{A})\right)_{\xi}$.

Remark 5.4. (1) Hypothesis 5.3 (A) may be regarded as a global analogue of [7, Lemma C.6]. It is also assumed in the theory of twisted automorphic descents. See Jiang-Zhang [32, Conjecture 2.3] and the remark after this conjecture for a nature of this expectation and some example.

(2) The main difficulty of Hypothesis $5.3(A)$ is the tempered-ness of $\pi^{\prime}$. Since all representations occurring in the space $\mathcal{S}_{(l-1) / 2}\left(\operatorname{Sp}_{1}(F) \backslash \widetilde{\operatorname{Sp}}_{1}(\mathbb{A})\right)$ have tempered A-parameters by Example B.9, Hypothesis 5.3 (A) is true when $r \leq 2$.

(3) Hypothesis 5.3 (B) means that the $L^{2}$-orthogonal complement of

$$
\left\{\varphi^{\prime} \cdot \Theta_{\xi}^{\phi} \mid \varphi^{\prime} \in \mathcal{S}_{(l-1) / 2}\left(\operatorname{Sp}_{r}(F) \backslash \widetilde{\operatorname{Sp}}_{r}(\mathbb{A})\right), \phi \in \mathcal{S}\left(X_{r}(\mathbb{A})\right)_{\xi}\right\}
$$

in $\mathcal{S}_{l / 2}\left(\operatorname{Sp}_{r}(F) \backslash \widetilde{\operatorname{Sp}}_{r}(\mathbb{A})\right)$ is equal to $\{0\}$. Theta liftings might have potential for Hypothesis $5.3(B)$.

(4) In the classical setting, Hypothesis $5.3(C)$ means the surjectivity of the $\xi$-th Fourier-Jacobi expansion of Siegel modular forms of degree $r+1$. For $r=1$, see Aoki [2] and IbukiyamaPoor-Yuen [24].

(5) In any case, Hypothesis 5.3 seems quite difficult. For the proof, it might be necessary to consider averages of Fourier-Jacobi periods (cf. see a paper of Nelson-Venkatesh [42]).

Using the GGP conjecture (Conjecture 5.2) and Hypothesis 5.3, we will show Conjecture 5.1. First, we consider the (almost) equal rank case, i.e., the case where $n=r$ or $n=r+1$.

Theorem 5.5. Assume the GGP conjecture (Conjecture 5.2) and Hypothesis 5.3 (A). Then for irreducible representations with tempered A-parameters, we have

$$
(M)_{r-1, r} \Longrightarrow(M)_{r, r} \Longrightarrow(M)_{r, r+1} \text {. }
$$


In particular, $(M)_{r, r}$ and $(M)_{r, r+1}$ for irreducible representations with tempered A-parameters are true for any $r \geq 0$.

Proof. First, we show $(M)_{r-1, r} \Longrightarrow(M)_{r, r}$. Let $\pi$ be an irreducible representation of $\widetilde{\operatorname{Sp}}_{r}\left(\mathbb{A}_{\mathrm{fin}}\right)$ occurring in $\mathcal{S}_{k+r}\left(\operatorname{Sp}_{r}(F) \backslash \operatorname{Sp}_{r}(\mathbb{A})\right)$ with tempered $A$-parameter $\Psi$. We choose a totally positive element $\xi \in F^{\times}$such that $\mathrm{FJ}_{\psi_{\xi}}(\pi) \neq 0$. By Hypothesis $5.3(\mathrm{~A})$, we can find an irreducible representation $\pi^{\prime}$ of $\widetilde{\operatorname{Sp}}_{r-1}\left(\mathbb{A}_{\text {fin }}\right)$ occurring in $\mathcal{S}_{k+r-1 / 2}\left(\operatorname{Sp}_{r-1}(F) \backslash \widetilde{\operatorname{Sp}}_{r-1}(\mathbb{A})\right)$ with tempered $A$-parameter $\Psi^{\prime}$ such that $\mathcal{P}_{r, r-1, \psi_{\xi}}$ is not identically zero on $\pi \times \overline{\pi^{\prime}} \times \mathcal{S}\left(X_{r-1}(\mathbb{A})\right)_{\xi}$.

Suppose that $\mathcal{M}_{\psi, \tau}^{(r)}(\pi) \neq 0$. Then by Proposition 4.8 (b), we see that $\mathcal{P}_{r, r, \psi_{\xi}}$ is not identically zero on $\pi \times \overline{\mathcal{M}_{\psi, \tau \chi_{\xi}}^{(r)}\left(\pi^{\prime}\right)} \times \mathcal{S}\left(X_{r}(\mathbb{A})\right)_{\xi}$. Since $\pi^{\prime}$ has a tempered $A$-parameter $\Psi^{\prime}$, by Theorem 4.5 and Proposition 4.3, the Miyawaki lifting $\mathcal{M}_{\psi, \tau \chi_{\xi}}^{(r)}\left(\pi^{\prime}\right)$ is irreducible and has a tempered $A$-parameter $\Psi^{\prime} \boxplus \tau \chi_{(-1)^{r-1} \xi}$. By the GGP conjecture (Conjecture $5.2(1)$ ), we conclude that

$$
L\left(1 / 2, \pi \times \pi^{\prime} \times \chi_{-\xi}\right) L\left(1 / 2, \pi \times\left(\tau \chi_{(-1)^{r-1} \xi}\right) \times \chi_{-\xi}\right) \neq 0,
$$

so that $L\left(1 / 2, \pi \times \tau \chi_{(-1)^{r}}\right) \neq 0$.

Conversely, suppose that $L\left(1 / 2, \pi \times \tau \chi_{(-1)^{r}}\right) \neq 0$. By $(M)_{r-1, r}$, we have $\mathcal{M}_{\psi, \tau \chi_{\xi}}^{(r)}\left(\pi^{\prime}\right) \neq 0$. Hence by Theorem 4.5 and Proposition 4.3, it is irreducible and has a tempered $A$-parameter $\Psi^{\prime} \boxplus \tau \chi_{(-1)^{r-1} \xi}$. By the GGP conjecture (Conjecture 5.2 (1), (2) and Theorem C.4), we see that $\mathcal{P}_{r, r, \psi_{\xi}}$ is not identically zero on $\pi \times \overline{\mathcal{M}_{\psi, \tau \chi_{\xi}}^{(r)}\left(\pi^{\prime}\right)} \times \mathcal{S}\left(X_{r}(\mathbb{A})\right)_{\xi}$. Hence by Proposition 4.8, we conclude that $\mathcal{M}_{\psi, \tau}^{(r)}(\pi) \neq 0$. This completes the proof of $(M)_{r-1, r} \Longrightarrow(M)_{r, r}$.

Next, we show $(M)_{r, r} \Longrightarrow(M)_{r, r+1}$. By Lemma B.10, there exists an irreducible representation $\Pi=\otimes_{v<\infty}^{\prime} \Pi_{v}$ of $\widetilde{\operatorname{Sp}}_{r+1}\left(\mathbb{A}_{\mathrm{fin}}\right)$ occurring in $\mathcal{S}_{k+r+1 / 2}\left(\operatorname{Sp}_{r+1}(F) \backslash \widetilde{\operatorname{Sp}}_{r+1}(\mathbb{A})\right)$ such that $\Pi_{v}=\mathcal{M}_{\psi_{v}, \tau_{v}}^{(r+1)}\left(\pi_{v}\right)$ for each $v<\infty$, and the $A$-parameter for $\Pi$ is equal to $\Psi \otimes \tau \chi_{-1}^{r}$. We choose a totally positive element $\xi \in F^{\times}$such that $\mathrm{FJ}_{\psi_{\xi}}(\Pi) \neq 0$. Using Hypothesis 5.3 (A), we take an irreducible representation $\pi^{\prime}$ of $\operatorname{Sp}_{r}\left(\mathbb{A}_{\text {fin }}\right)$ occurring in $\mathcal{S}_{k+r}\left(\operatorname{Sp}_{r}(F) \backslash \widetilde{\operatorname{Sp}}_{r}(\mathbb{A})\right)$ with tempered $A$-parameter $\Psi^{\prime}$ such that $\mathcal{P}_{r+1, r, \psi_{\xi}}$ is not identically zero on $\Pi \times \overline{\pi^{\prime}} \times \mathcal{S}\left(X_{r}(\mathbb{A})\right)_{\xi}$. By the GGP conjecture (Conjecture $5.2(1)$ ), we see that

$$
L\left(1 / 2, \Pi \times \pi^{\prime} \times \chi_{\xi}\right)=L\left(1 / 2, \pi \times \pi^{\prime} \times \chi_{\xi}\right) L\left(1 / 2, \pi^{\prime} \times \tau \chi_{(-1)^{r} \xi}\right)
$$

is nonzero. Since $L\left(1 / 2, \pi^{\prime} \times \tau \chi_{(-1)^{r} \xi}\right) \neq 0$, by $(M)_{r, r}$, we see that $\mathcal{M}_{\psi, \tau \chi_{\xi}}^{(r)}\left(\pi^{\prime}\right)$ is nonzero. By Corollary $4.6(1)$, it is equal to $\pi^{\prime}$ itself. Since $L\left(1 / 2, \pi \times \pi^{\prime} \times \chi_{\xi}\right) \neq 0$, by the GGP conjecture (Conjecture 5.2 (2) and Theorem C.4), we see that $\mathcal{P}_{r, r, \psi_{\xi}}$ is not identically zero on

$$
\pi \times \overline{\pi^{\prime}} \times \mathcal{S}\left(X_{r}(\mathbb{A})\right)_{\xi}=\pi \times \overline{\mathcal{M}_{\psi, \tau \chi \xi}^{(r)}\left(\pi^{\prime}\right)} \times \mathcal{S}\left(X_{r}(\mathbb{A})\right)_{\xi} .
$$

Then by the seesaw identity (Proposition $4.7(2)$ ), we see that $\mathcal{P}_{r+1, r, \psi_{\xi}}$ is not identically zero on $\mathcal{M}_{\psi, \tau}^{(r+1)}(\pi) \times \overline{\pi^{\prime}} \times \mathcal{S}\left(X_{r}(\mathbb{A})\right)_{\xi}$. In particular we have $\mathcal{M}_{\psi, \tau}^{(r+1)}(\pi) \neq 0$. This completes the proof of $(M)_{r, r} \Longrightarrow(M)_{r, r+1}$.

Corollary 5.6. The conjecture $(M)_{1,1}$ is true.

Proof. Note that any irreducible representation occurring in $\mathcal{S}_{k+1}\left(\operatorname{Sp}_{1}(F) \backslash \operatorname{Sp}_{1}(\mathbb{A})\right)$ has a tempered $A$-parameter (Remark B.9). The conjecture $(M)_{0,1}$ and Hypothesis 5.3 (A) for $r=1$ are trivial. 
The GGP conjecture for $n=n^{\prime}=1$ is known (see e.g., [12, Proof of Theorem 7.1], [44, Proposition 4.1, Theorem 4.5] and [56]). Hence we have $(M)_{1,1}$.

Next, we consider the going-up case, i.e., the case where $n>r+1$.

Theorem 5.7. Suppose that $n \geq r+2$.

(1) Assuming Hypothesis 5.3 (B), we have

$$
(M)_{r, n-1} \Longrightarrow(M)_{r, n}
$$

(2) Assuming Hypothesis $5.3(C)$, we have

$$
(M)_{r+1, n} \Longrightarrow(M)_{r, n} \text {. }
$$

In particular, assuming one of Hypothesis $5.3(B)$ or $(C)$, the conjecture $(M)_{r, n}$ for $n \geq r+1$ is reduced to $(M)_{r, r+1}$ or $(M)_{n-1, n}$.

Proof. We prove only the first assertion. The proof of the second one is similar.

Let $\pi$ be an irreducible representation of $\widetilde{\operatorname{Sp}}_{r}\left(\mathbb{A}_{\text {fin }}\right)$ occurring in $\mathcal{S}_{k+(n+r) / 2}\left(\operatorname{Sp}_{r}(F) \backslash \widetilde{\operatorname{Sp}_{r}}(\mathbb{A})\right)$. Using Hypothesis $5.3(\mathrm{~B})$, we choose an irreducible representation $\pi^{\prime}$ of $\widetilde{\operatorname{Sp}}_{r}\left(\mathbb{A}_{\text {fin }}\right)$ which appears in $\mathcal{S}_{k+(n+r-1) / 2}\left(\operatorname{Sp}_{r}(F) \backslash \widetilde{\operatorname{Sp}}_{r}(\mathbb{A})\right)$ such that $\mathcal{P}_{r, r, \psi_{\xi}}$ is not identically zero on $\pi \times \overline{\pi^{\prime}} \times \mathcal{S}\left(X_{r}(\mathbb{A})\right)_{\xi}$. Applying $(M)_{r, n-1}$ to $\pi^{\prime}$, we have $\Pi^{\prime}=\mathcal{M}_{\psi, \tau \chi_{\xi}}^{(n-1)}\left(\pi^{\prime}\right) \neq 0$. By Proposition 4.4, we have $\pi^{\prime} \subset$ $\mathcal{M}_{\psi, \tau \chi_{\xi}}^{(r)}\left(\Pi^{\prime}\right)$. Hence $\mathcal{P}_{r, r, \psi_{\xi}}$ is not identically zero on $\pi \times \overline{\mathcal{M}_{\psi, \tau \chi_{\xi}}^{(r)}\left(\Pi^{\prime}\right)} \times \mathcal{S}\left(X_{r}(\mathbb{A})\right)_{\xi}$. By the seesaw identity (Proposition $4.7(2)$ ), we see that $\mathcal{P}_{n, n-1, \psi_{\xi}}$ is not identically zero on $\mathcal{M}_{\psi, \tau}^{(n)}(\pi) \times \overline{\Pi^{\prime}} \times$ $\mathcal{S}\left(X_{n-1}(\mathbb{A})\right)_{\xi}$. In particular, we have $\mathcal{M}_{\psi, \tau}^{(n)}(\pi) \neq 0$.

\section{Appendix A. JaCquet modules of RePRESEntations of Metaplectic Groups}

Let $F$ be a non-archimedean local field of characteristic zero. In this appendix, we recall computations of Jacquet modules of induced representations of $\mathrm{GL}_{k}(F)$ or $\widetilde{\operatorname{Sp}}_{n}(F)$.

A.1. Induced representations of general linear groups. Let $P(F)=M(F) N(F)$ be a parabolic subgroup of $\mathrm{GL}_{k}(F)$ containing the Borel subgroup consisting of upper triangular matrices. Then the Levi part $M(F)$ is of the form $\mathrm{GL}_{k_{1}}(F) \times \cdots \times \mathrm{GL}_{k_{l}}(F)$ with $k_{1}+\cdots+k_{l}=k$. For representations $\tau_{1}, \ldots, \tau_{l}$ of $\mathrm{GL}_{k_{1}}(F), \ldots, \mathrm{GL}_{k_{l}}(F)$, respectively, we denote the normalized induced representation by

$$
\tau_{1} \times \cdots \times \tau_{l}=\operatorname{Ind}_{P(F)}^{\mathrm{GL}_{k}(F)}\left(\tau_{1} \otimes \cdots \otimes \tau_{l}\right) .
$$

A segment is a symbol $[x, y]$, where $x, y \in \mathbb{R}$ with $x-y \in \mathbb{Z}$. We identify $[x, y]$ as the set $\{x, x-1, \ldots, y\}$ if $x \geq y$, and $\{x, x+1, \ldots, y\}$ if $x \leq y$. Let $\rho$ be an irreducible unitary supercuspidal representation of $\mathrm{GL}_{d}(F)$. Then the normalized induced representation

$$
\rho|\cdot|{ }^{x} \times \cdots \times \rho|\cdot|^{y}
$$

has a unique irreducible subrepresentation, which is denoted by

$$
\langle\rho ; x, \ldots, y\rangle \text {. }
$$

If $x \geq y$, this is called a Steinberg representation, which is an essentially discrete series representation of $\mathrm{GL}_{d(|x-y|+1)}(F)$. If $x<y$, this is called a Speh representation. For example, if $\rho=\mu$ be a unitary character (i.e., $d=1$ ) and $x<y$, then $\langle\mu ; x, \ldots, y\rangle=\mu\left|\operatorname{det}_{y-x+1}\right|^{(x+y) / 2}$ is a character of $\mathrm{GL}_{y-x+1}(F)$, where we denote by $\operatorname{det}_{k}$ the determinant character of $\mathrm{GL}_{k}(F)$.

Definition A.1. Let $[x, y]$ and $\left[x^{\prime}, y^{\prime}\right]$ be two segments. 
(1) When $(x-y)\left(x-y^{\prime}\right) \geq 0$, we say that $[x, y]$ and $\left[x^{\prime}, y^{\prime}\right]$ are linked if $[x, y] \not \subset\left[x^{\prime}, y^{\prime}\right],\left[x^{\prime}, y^{\prime}\right] \not \subset$ $[x, y]$ as sets, and $[x, y] \cup\left[x, y^{\prime}\right]$ is also a segment.

(2) When $(x-y)\left(x^{\prime}-y^{\prime}\right)<0$, we say that $[x, y]$ and $\left[x^{\prime}, y^{\prime}\right]$ are linked if $[y, x]$ and $\left[x^{\prime}, y^{\prime}\right]$ are linked, and $x, y \notin\left[x^{\prime}, y^{\prime}\right]$ and $x^{\prime}, y^{\prime} \notin[x, y]$.

The linked-ness gives an irreducibility criterion for induced representations.

Theorem A.2 (Zelevinsky [59, Theorems 4.2, 9.7], Møglin-Waldspurger [41]). Let $[x, y]$ and $\left[x^{\prime}, y^{\prime}\right]$ be segments, and let $\rho$ and $\rho^{\prime}$ be irreducible unitary supercuspidal representations of $\mathrm{GL}_{d}(F)$ and $\mathrm{GL}_{d^{\prime}}(F)$, respectively. Then the induced representation

$$
\langle\rho ; x, \ldots, y\rangle \times\left\langle\rho^{\prime} ; x^{\prime}, \ldots, y^{\prime}\right\rangle
$$

is irreducible unless $[x, y]$ are $\left[x^{\prime}, y^{\prime}\right]$ are linked, and $\rho \cong \rho^{\prime}$.

For a partition $\left(k_{1}, k_{2}\right)$ of $k$, we denote by $R_{\left(k_{1}, k_{2}\right)}$ the normalized Jacquet functor of representations of $\mathrm{GL}_{k}(F)$ with respect to the standard maximal parabolic subgroup $P(F)=M(F) N(F)$ with $M(F) \cong \mathrm{GL}_{k_{1}}(F) \times \mathrm{GL}_{k_{2}}(F)$. The Jacquet module of $\langle\rho ; x, \ldots, y\rangle$ is computed by Zelevinsky.

Proposition A.3 ([59, Propositions 3.4, 9.5]). Let $\rho$ be an irreducible unitary supercuspidal representation of $\mathrm{GL}_{d}(F)$. Suppose that $x \neq y$ and set $k=d(|x-y|+1)$. Then $R_{\left(k_{1}, k_{2}\right)}(\langle\rho ; x, \ldots, y\rangle)=0$ unless $k_{1} \equiv 0 \bmod d$. If $k_{1}=d m$ with $1 \leq m \leq|x-y|$, we have

$$
R_{\left(k_{1}, k_{2}\right)}(\langle\rho ; x, \ldots, y\rangle)=\langle\rho ; x, \ldots, x-\epsilon(m-1)\rangle \otimes\langle\rho ; x-\epsilon m, \ldots, y\rangle,
$$

where $\epsilon \in\{ \pm 1\}$ is defined so that $\epsilon(x-y)>0$.

A.2. Representations of double covers of general linear groups. Recall that for $a \in F^{\times}$, the Weil constant $\alpha_{\psi}(a)$ is an eighth root of unity, and satisfies that

$$
\frac{\alpha_{\psi}(a) \alpha_{\psi}(b)}{\alpha_{\psi}(1) \alpha_{\psi}(a b)}=\langle a, b\rangle
$$

for $a, b \in F$, where the right hand side is the Hilbert symbol. In particular,

$$
\left(\frac{\alpha_{\psi}(1)}{\alpha_{\psi}(a)}\right)^{2}=\chi_{-1}(a)
$$

for $a \in F^{\times}$, where $\chi_{-1}$ is the quadratic character associated to $F(\sqrt{-1}) / F$.

A double cover of $\mathrm{GL}_{k}(F)$ is given by

$$
\widetilde{\mathrm{GL}}_{k}(F)=\mathrm{GL}_{k}(F) \times\{ \pm 1\}
$$

with group law

$$
\left(g_{1}, \epsilon_{1}\right) \cdot\left(g_{2}, \epsilon_{2}\right)=\left(g_{1} g_{2}, \epsilon_{1} \epsilon_{2} \cdot\left\langle\operatorname{det} g_{1}, \operatorname{det} g_{2}\right\rangle\right)
$$

Let $\operatorname{Irr}\left(\mathrm{GL}_{k}(F)\right)$ (resp. $\left.\operatorname{Irr}\left(\widetilde{\mathrm{GL}}_{k}(F)\right)\right)$ be the set of equivalence classes of irreducible representations of $\mathrm{GL}_{k}(F)$ (resp. the set of equivalence classes of irreducible genuine representations of $\widetilde{\mathrm{GL}}_{k}(F)$ ). For an irreducible representation $\tau$ of $\mathrm{GL}_{k}(F)$ and $(g, \epsilon) \in \widetilde{\mathrm{GL}}_{k}(F)$, we set

$$
\tau_{\psi}(g, \epsilon)=\epsilon \frac{\alpha_{\psi}(1)}{\alpha_{\psi}(\operatorname{det} g)} \tau(g) .
$$

Then $\tau_{\psi}$ is irreducible and genuine, and the map $\tau \mapsto \tau_{\psi}$ gives a bijection $\operatorname{Irr}\left(\mathrm{GL}_{k}(F)\right) \rightarrow \operatorname{Irr}\left(\widetilde{\mathrm{GL}}_{k}(F)\right)$. For a more precise representation theory for $\widetilde{\mathrm{GL}}_{k}(F)$, see $[20, \S 4.1]$. 
A.3. Induced representations of symplectic and metaplectic groups. We denote by $R_{P_{t}(F)}$ the normalized Jacquet functor of representations of $\widetilde{\mathrm{Sp}}_{r}(F)$ with respect to the maximal parabolic subgroup $\widetilde{P}_{t}(F)$. For a smooth representation $\Pi$ of $\widetilde{\mathrm{Sp}}_{r}(F)$, we write s.s.( $\left.\Pi\right)$ for the semisimplification of $\Pi$.

Proposition A.4. Let $\pi$ be a representation of $\widetilde{\operatorname{Sp}_{r}}(F)$, $\mu$ be a unitary character of $F^{\times}$, and $\alpha \in \mathbb{C}$. Then s.s. $R_{P_{t}(F)}\left(\mu\left|\operatorname{det}_{k}\right|^{\alpha} \rtimes \pi\right)$ is isomorphic to the direct sum of

$$
\text { s.s. }\left(\mu^{-1}\left|\operatorname{det}_{k-a}\right|^{-\alpha-\frac{a}{2}} \times \mu\left|\operatorname{det}_{b}\right|^{\alpha-\frac{k-b}{2}} \times \tau_{\lambda}\right) \otimes\left(\mu\left|\operatorname{det}_{a-b}\right|^{\alpha-\frac{k-a-b}{2}} \rtimes \pi_{\lambda}\right),
$$

where $(a, b)$ runs over the pairs of integers such that $0 \leq b \leq a \leq k$ and $a-b \geq k-t$, and $\tau_{\lambda} \otimes \pi_{\lambda}$ runs over all irreducible subquotients of $R_{P_{a-b-k+t}(F)}(\pi)$ (with multiplicity).

Proof. This follows from Tadić's formula [51], [20, Proposition 4,5].

\section{Appendix B. Local Langlands CORRespondence And ARThur's multiplicity formula}

In this appendix, we summarize the local Langlands correspondence and Arthur's multiplicity formula.

B.1. Local Langlands correspondence. We recall the local Langlands correspondence (LLC) for $\operatorname{Sp}_{n}(F)$ and $\widetilde{\operatorname{Sp}}_{n}(F)$. Let $F$ be a local field of characteristic zero. Fix a non-trivial unitary character $\psi$ of $F$. We denote by $W_{F}$ and $W D_{F}$ the Weil group and the Weil-Deligne group of $F$, respectively, i.e.,

$$
W D_{F}= \begin{cases}W_{F} \times \mathrm{SL}_{2}(\mathbb{C}) & \text { if } F \text { is non-archimedean, } \\ W_{F} & \text { if } F \text { is archimedean. }\end{cases}
$$

A representation of $W D_{F}$ is a homomorphism $\phi: W D_{F} \rightarrow \mathrm{GL}_{N}(\mathbb{C})$ such that

- $\phi($ Frob) is semi-simple if $F$ is non-archimedean;

- $\phi \mid W_{F}$ is smooth if $F$ is non-archimedean, and $\phi$ is continuous if $F$ is archimedean;

- $\phi \mid \mathrm{SL}_{2}(\mathbb{C})$ is algebraic.

Here, Frob $\in W_{F}$ is a (geometric) Frobenius element if $F$ is non-archimedean. When $F$ is a nonarchimedean local field of residue characteristic $p>2$, we call a representation $\phi$ of $W D_{F}$ unramified if $\phi$ is trivial on $I_{F} \times \mathrm{SL}_{2}(\mathbb{C})$, where $I_{F}$ is the inertia subgroup of $W_{F}$.

Set

$$
\begin{aligned}
& \Phi\left(\operatorname{Sp}_{n}(F)\right)=\left\{\phi: W D_{F} \rightarrow \mathrm{SO}_{2 n+1}(\mathbb{C})\right\} / \cong \\
& \Phi\left(\widetilde{\operatorname{Sp}}_{n}(F)\right)=\left\{\phi: W D_{F} \rightarrow \operatorname{Sp}_{n}(\mathbb{C})\right\} / \cong
\end{aligned}
$$

For $G_{n}=\operatorname{Sp}_{n}$ or $G_{n}={\widetilde{\mathrm{Sp}_{n}}}_{n}$, we call an element in $\Phi\left(G_{n}(F)\right)$ an $L$-parameter for $G(F)$. When $G_{n}=\operatorname{Sp}_{n}\left(\right.$ resp. $\left.G_{n}={\widetilde{\mathrm{Sp}_{n}}}\right)$, any $\phi \in \Phi\left(G_{n}(F)\right)$ can be decomposed into a direct sum

$$
\phi=m_{1} \phi_{1} \oplus \cdots \oplus m_{t} \phi_{t} \oplus \phi^{\prime} \oplus\left(\phi^{\prime}\right)^{\vee},
$$

where $\phi_{1}, \ldots, \phi_{t}$ are distinct irreducible orthogonal (resp. symplectic) representations of $W D_{F}, m_{i}$ is the multiplicity of $\phi_{i}$ in $\phi$, and $\phi^{\prime}$ is a sum of irreducible representations of $W D_{F}$ which are not orthogonal (resp. symplectic). We define the component group $A_{\phi}$ of $\phi$ by

$$
A_{\phi}=\bigoplus_{i=1}^{t}(\mathbb{Z} / 2 \mathbb{Z}) a_{i}
$$


Namely, $A_{\phi}$ is a free $\mathbb{Z} / 2 \mathbb{Z}$-module of rank $t$, and $\left\{a_{1}, \ldots, a_{t}\right\}$ is a basis of $A_{\phi}$ with $a_{i}$ associated to $\phi_{i}$. For $a=a_{i_{1}}+\cdots+a_{i_{k}} \in A_{\phi}$ with $1 \leq i_{1}<\cdots<i_{k} \leq t$, put

$$
\phi^{a}=\phi_{i_{1}} \oplus \cdots \oplus \phi_{i_{k}} .
$$

We call $z_{\phi}=\sum_{i=1}^{t} m_{i} a_{i} \in A_{\phi}$ the central element of $A_{\phi}$. We denote the Pontryagin dual of $A_{\phi}$ by $\widehat{A_{\phi}}=\left\{\eta: A_{\phi} \rightarrow\{ \pm 1\}\right\}$.

For $\phi \in \Phi\left(\operatorname{Sp}_{n}(F)\right)$ (resp. $\phi \in \Phi\left(\widetilde{\operatorname{Sp}}_{n}(F)\right)$ ), we say that:

- $\phi$ is of good parity if $\phi$ is a direct sum of irreducible orthogonal (resp. symplectic) representations;

- $\phi$ is tempered if $\phi\left(W_{F}\right)$ is bounded;

- $\phi$ is almost tempered if each irreducible constituent $\phi_{i}$ of $\phi$ is of the form $\phi_{i}=\phi_{i}^{\prime}|\cdot|{ }^{s_{i}}$ such that $\phi_{i}^{\prime}\left(W_{F}\right)$ is bounded and $-1 / 2<s_{i}<1 / 2$.

The set of equivalence classes of irreducible representations of $\widetilde{\operatorname{Sp}_{n}}(F)$ which are genuine (resp. not genuine) is denoted by $\operatorname{Irr}\left(\widetilde{\operatorname{Sp}}_{n}(F)\right)$ (resp. $\left.\operatorname{Irr}\left(\operatorname{Sp}_{n}(F)\right)\right)$. The LLC classifies $\operatorname{Irr}\left(G_{n}(F)\right)$ by $\Phi\left(G_{n}(F)\right)$ for $G_{n}=\widetilde{\mathrm{Sp}}_{n}$ or $G_{n}=\mathrm{Sp}_{n}$.

Theorem B.1 $([37,1,3,15])$. Let $G_{n}={\widetilde{\mathrm{Sp}_{n}}}_{\text {or }} G_{n}=\mathrm{Sp}_{n}$.

(1) There is a canonical surjective map

$$
\operatorname{Irr}\left(G_{n}(F)\right) \rightarrow \Phi\left(G_{n}(F)\right) .
$$

For $\phi \in \Phi\left(G_{n}(F)\right)$, we denote the inverse image of $\phi$ by $\Pi_{\phi}$, and call it the L-packet of $\phi$.

(2) There exists an injective map

$$
\Pi_{\phi} \hookrightarrow \widehat{A_{\phi}} .
$$

This is surjective if $G_{n}=\widetilde{\mathrm{Sp}}_{n}$. When $G_{n}=\mathrm{Sp}_{n}$, the image of this map is equal to

$$
\left\{\eta \in \widehat{A_{\phi}} \mid \eta\left(z_{\phi}\right)=1\right\} \text {. }
$$

When $\pi \in \Pi_{\phi}$ corresponds to $\eta \in \widehat{A_{\phi}}$, we call the pair $(\phi, \eta)$ the L-parameter for $\pi$.

(3) When $F$ is a non-archimedean local field of residue characteristic $p>2$, an irreducible representation $\pi$ is unramified if and only if its L-parameter $(\phi, \eta)$ satisfies that $\phi$ is unramified and $\eta=\mathbf{1}$.

(4) $\pi \in \Pi_{\phi}$ is (almost) tempered if and only if $\phi$ is (almost) tempered.

(5) If $\pi \in \Pi_{\phi}$ is discrete series, then $\phi \in \Phi\left(G_{n}(F)\right)$ is of good parity.

(6) If $\phi$ is tempered, then one can decompose

$$
\phi=\phi_{\tau} \oplus \phi_{0} \oplus \phi_{\tau}^{\vee}
$$

where

- $\phi_{0} \in \Phi\left(G_{n_{0}}(F)\right)$ is of good parity;

- $\phi_{\tau}$ is a sum of irreducible representations which are not orthogonal when $G_{n}=\operatorname{Sp}_{n}(F)$, and are not symplectic when $G_{n}=\widetilde{\operatorname{Sp}}_{n}(F)$.

Let $\tau$ be the irreducible (tempered) representation of $\mathrm{GL}_{k}(F)$ corresponding to $\phi_{\tau}$. Then for $\pi_{0} \in \Pi_{\phi_{0}}$, the induced representation $\tau \rtimes \pi_{0}$ is irreducible, and the L-packet $\Pi_{\phi}$ is given by

$$
\Pi_{\phi}=\left\{\tau \rtimes \pi_{0} \mid \pi_{0} \in \Pi_{\phi_{0}}\right\} .
$$

If the $L$-parameter for $\pi_{0}$ is $\left(\phi_{0}, \eta_{0}\right)$, then the one for $\tau \rtimes \pi_{0}$ is $\left(\phi, \eta_{0}\right)$, where we regard $\eta_{0}$ as a character of $A_{\phi}$ via the canonical identification $A_{\phi}=A_{\phi_{0}}$. 
(7) If $\pi=J\left(\tau_{1}|\cdot|{ }^{s_{1}}, \ldots, \tau_{t}|\cdot|{ }^{s_{t}}, \pi_{0}\right)$, then the L-parameter $(\phi, \eta)$ for $\pi$ is given by

$$
\phi=\phi_{1}|\cdot|^{s_{1}} \oplus \cdots \oplus \phi_{t}|\cdot|^{s_{t}} \oplus \phi_{0} \oplus \phi_{t}^{\vee}|\cdot|^{-s_{t}} \oplus \cdots \oplus \phi_{1}^{\vee}|\cdot|^{-s_{1}}
$$

and $\eta=\eta_{0}$, where $\left(\phi_{0}, \eta_{0}\right)$ is the L-parameter for $\pi_{0}$, and $\phi_{i}$ is the (tempered) representation of $W D_{F}$ corresponding to $\tau_{i}$ for $i=1, \ldots, t$.

B.2. Irreducibility criterion for standard modules. In this subsection, we assume that $F$ is non-archimedean. For each representation $\phi$ of $W D_{F}$, one can consider the $L$-function $L(s, \phi)$ attached to $\phi$. We recall a criterion for the irreducibility of standard modules in terms of analytic properties of $L$-functions.

Proposition B.2. Let $\pi_{0}\left(\right.$ resp. $\left.\tau_{i}\right)$ be an irreducible tempered representation of $\widetilde{\operatorname{Sp}}_{r_{0}}(F)\left(r e s p . \mathrm{GL}_{k_{i}}(F)\right)$ with the L-parameter $\left(\phi_{0}, \eta_{0}\right)$ (resp. with associated representation $\phi_{i}$ of $\left.W D_{F}\right)$, and $s_{1}, \ldots, s_{t}$ be real numbers such that $s_{1}>\cdots>s_{t}>0$. Assume the following:

- If $\pi_{0}$ is not genuine, then

$$
\begin{aligned}
& \left(\prod_{i=1}^{t} L\left(s-2 s_{i}, \phi_{i}^{\vee}, \wedge^{2}\right) L\left(s-s_{i}, \phi_{0} \otimes \phi_{i}^{\vee}\right)\right) \\
& \times\left(\prod_{1 \leq i<j \leq t} L\left(s-s_{i}+s_{j}, \phi_{i}^{\vee} \otimes \phi_{j}\right) L\left(s-s_{i}-s_{j}, \phi_{i}^{\vee} \otimes \phi_{j}^{\vee}\right)\right)
\end{aligned}
$$

is regular at $s=1$.

- If $\pi_{0}$ is genuine, then

$$
\begin{aligned}
& \left(\prod_{i=1}^{t} L\left(s-2 s_{i}, \phi_{i}^{\vee}, \operatorname{Sym}^{2}\right) L\left(s-s_{i}, \phi_{0} \otimes \phi_{i}^{\vee}\right)\right) \\
& \times\left(\prod_{1 \leq i<j \leq t} L\left(s-s_{i}+s_{j}, \phi_{i}^{\vee} \otimes \phi_{j}\right) L\left(s-s_{i}-s_{j}, \phi_{i}^{\vee} \otimes \phi_{j}^{\vee}\right)\right)
\end{aligned}
$$

is regular at $s=1$.

Then the standard module $\tau_{1}|\cdot|{ }^{s_{1}} \times \cdots \times \tau_{t}|\cdot|{ }^{s_{t}} \rtimes \pi_{0}$ is irreducible.

Proof. Note that our assumptions are equivalent that the adjoint $L$-function $L(s, \phi, \mathrm{Ad})$ is regular at $s=1$, where $(\phi, \eta)$ is the $L$-parameter for $J\left(\tau_{1}|\cdot|{ }^{s_{1}}, \ldots,\left.\tau_{t}|\cdot|\right|^{s_{t}}, \pi_{0}\right)$. The non-genuine case is a result of Heiermann [23] together with a conjecture of Gross-Prasad-Rallis (proven in [13, Appendix B]). The genuine case is [5, Theorem 3.13].

This proposition has several corollaries.

Corollary B.3. If $\pi$ is irreducible and almost tempered, then $\pi$ is isomorphic to an irreducible standard module.

Proof. This immediately follows from Proposition B.2.

Corollary B.4. Let $\mu$ be a unitary character of $F^{\times}$. Set $\delta=0$ if $G_{n}=\operatorname{Sp}_{n}(F)$, and $\delta=1$ if $G_{n}=\widetilde{\operatorname{Sp}}_{n}(F)$. Suppose that $\phi \in \Phi\left(G_{n}(F)\right)$ is of good parity. Then for any $\pi \in \Pi_{\phi}$ and for any positive integer a with $a \not \equiv \delta \bmod 2$, the standard module

$$
\mu|\cdot|^{\frac{a}{2}} \rtimes \pi
$$

is irreducible. 
Proof. Since $\phi$ is of good parity and $a \not \equiv \delta \bmod 2$, we see that $L\left(s-a / 2, \phi \otimes \mu^{-1}\right)$ is regular at $s=1$. If $\delta=0$, then $L\left(s-a, \mu^{-1}, \wedge^{2}\right)=1$, which is entire. If $\delta=1$, then $L\left(s-a, \mu^{-1}, \operatorname{Sym}^{2}\right)=L\left(s-a, \mu^{-2}\right)$, which is regular at $s=1$ when $a \neq 1$. By Proposition B.2, we obtain the corollary.

Corollary B.5. Let $\pi \in \Pi_{\phi}$ with $\phi$ of good parity, If s.s. $R_{P_{1}(F)}(\pi)$ contains $\chi|\cdot|{ }^{\alpha} \otimes \pi_{0}$ for some unitary character $\chi$ and $\alpha \in \mathbb{R}$, then $2 \alpha \in \mathbb{Z}$ and $2 \alpha \equiv \delta \bmod 2$.

Proof. First, we assume that $\pi$ is discrete series. Replacing $\pi_{0}$ if necessary, we may assume that

$$
\pi \hookrightarrow \chi|\cdot|^{\alpha} \rtimes \pi_{0} .
$$

By Casselman's criterion (see e.g., [34, Lemma 2.4]), we see that

- $\alpha>0$

- $\pi_{0}$ is discrete series;

- the standard module $\chi|\cdot|^{\alpha} \rtimes \pi_{0}$ is reducible.

In particular, if $\pi_{0} \in \Pi_{\phi_{0}}$, then $L\left(s-\alpha, \phi_{0} \otimes \chi^{-1}\right)$ has a pole at $s=1$, or $L\left(s-2 \alpha, \chi^{-1}, \wedge^{2}\right)$ (resp. $L\left(s-2 \alpha, \chi^{-1}, \mathrm{Sym}^{2}\right)$ ) has a pole at $s=1$ when $\delta=0(\operatorname{resp} . \delta=1)$. Since $\phi_{0}$ is of good parity, this condition implies that $2 \alpha \in \mathbb{Z}$ and $2 \alpha \equiv \delta \bmod 2$.

In general, if $\pi \in \Pi_{\phi}$ with $\phi$ of good parity, then

$$
\pi \hookrightarrow \tau_{1} \times \cdots \times \tau_{r} \rtimes \pi_{0},
$$

where

- $\tau_{i}$ is an irreducible discrete series representation of $\mathrm{GL}_{k_{i}}(F)$ to which the irreducible representation $\phi_{i}$ of $W D_{F}$ corresponding is orthogonal if $\delta=0$, and symplectic if $\delta=1$;

- $\pi_{0}$ is an irreducible discrete series representation of ${\widetilde{\mathrm{Sp}_{n}}}_{n_{0}}(F)$.

Then the assertion follows from Tadić's formula [51] [20, Proposition 4,5].

B.3. Lowest weight modules. In this subsection, we assume that $F=\mathbb{R}$ and $\psi(x)=\exp (2 \pi a \sqrt{-1} x)$ for some $a>0$. Recall that we denote the irreducible lowest weight representation of $\widetilde{\mathrm{Sp}}_{n}(\mathbb{R})$ with lowest $\widetilde{K}_{\infty}$-type $\operatorname{det}^{l / 2}$ by $\mathcal{D}_{l / 2}^{(n)}$. We determine the $L$-parameters for $\mathcal{D}_{l / 2}^{(n)}$ when it is discrete series, which is equivalent that $l>2 n$. Note that the infinitesimal character of $\mathcal{D}_{l / 2}^{(n)}$ is equal to

$$
\left(\frac{l}{2}-1, \frac{l}{2}-2, \ldots, \frac{l}{2}-n\right)
$$

Recall that the Weil group $W_{\mathbb{R}}$ of $\mathbb{R}$ is of the form

$$
W_{\mathbb{R}}=\mathbb{C}^{\times} \cup \mathbb{C}^{\times} j
$$

with the group low

$$
j^{2}=-1, \quad j z j^{-1}=\bar{z}
$$

for $z \in \mathbb{C}^{\times}$. Let sgn be the sign character of $W_{\mathbb{R}}$ defined by $\operatorname{sgn}(j)=-1$ and $\operatorname{sgn}(z)=1$ for $z \in \mathbb{C}^{\times}$. For each positive integer $k$, we define a 2-dimensional irreducible representation $\rho_{k}: W_{\mathbb{R}} \rightarrow \mathrm{GL}_{2}(\mathbb{C})$ by

$$
\rho_{k}(j)=\left(\begin{array}{cc}
0 & (-1)^{k} \\
1 & 0
\end{array}\right), \quad \rho_{k}\left(r e^{\sqrt{-1} \theta}\right)=\left(\begin{array}{cc}
e^{k \sqrt{-1} \theta} & 0 \\
0 & e^{-k \sqrt{-1} \theta}
\end{array}\right)
$$

for $r>0$ and $\theta \in \mathbb{R} / 2 \pi \mathbb{Z}$. Note that (a conjugate of) the image of $\rho_{k}$ is contained in $\mathrm{O}(2, \mathbb{C})$ if $k$ is even, and in $\mathrm{SL}_{2}(\mathbb{C})$ if $k$ is odd. 
For an integer $l>2 n$, we put

$$
\phi_{l}^{(n)}= \begin{cases}\rho_{l-2} \oplus \rho_{l-4} \oplus \cdots \oplus \rho_{l-2 n} \oplus \operatorname{sgn}^{n} & \text { if } l \equiv 0 \bmod 2, \\ \rho_{l-2} \oplus \rho_{l-4} \oplus \cdots \oplus \rho_{l-2 n} & \text { if } l \equiv 1 \bmod 2 .\end{cases}
$$

Then $\phi_{l}^{(n)} \in \Phi\left(\operatorname{Sp}_{n}(\mathbb{R})\right)$ if $l$ is even, and $\phi_{l}^{(n)} \in \Phi\left(\widetilde{\operatorname{Sp}_{n}}(\mathbb{R})\right)$ if $l$ is odd. When $\phi=\phi_{l}^{(n)}$, the component group $A_{\phi}$ is given by

$$
A_{\phi}=\left((\mathbb{Z} / 2 \mathbb{Z}) e_{l-2} \oplus(\mathbb{Z} / 2 \mathbb{Z}) e_{l-4} \oplus \cdots \oplus(\mathbb{Z} / 2 \mathbb{Z}) e_{l-2 n}\right)+(\mathbb{Z} / 2 \mathbb{Z}) z_{\phi},
$$

where $e_{k} \in A_{\phi}$ is the element associated to $\rho_{k} \subset \phi$.

Proposition B.6. Suppose that $l>2 n$. Then the L-parameter for $\mathcal{D}_{l / 2}^{(n)}$ is equal to $\left(\phi_{l}^{(n)}, \eta_{l}^{(n)}\right)$, where $\eta_{l}^{(n)}$ is determined by $\eta_{l}^{(n)}\left(e_{l-2 i}\right)=(-1)^{i-1}$ for $i=1, \ldots, n$.

Proof. Set $\phi=\phi_{l}^{(n)}$. Then the $L$-packet $\Pi_{\phi}$ is the set of discrete series representations with infinitesimal character

$$
\left(\frac{l}{2}-1, \frac{l}{2}-2, \ldots, \frac{l}{2}-n\right)
$$

Hence $\mathcal{D}_{l / 2}^{(n)} \in \Pi_{\phi}$. The description of $\eta_{l}^{(n)}$ can be obtained by a similar way to $[6$, Appendix A] using Schmid's character identity ([46, (9.4) Theorem] and [47, Theorem (b)]). We omit the detail.

B.4. Arthur's multiplicity formula. In this subsection, we let $F$ be a totally real number field, and $\psi$ be a non-trivial unitary character of $\mathbb{A} / F$. A discrete global $A$-parameter for $\operatorname{Sp}_{n}(F)$ (resp. $\left.\widetilde{\operatorname{Sp}}_{n}(F)\right)$ is a symbol

where

$$
\Psi=\tau_{1}\left[d_{1}\right] \boxplus \cdots \boxplus \tau_{t}\left[d_{t}\right]
$$

- $\tau_{i}$ is an irreducible cuspidal unitary automorphic representation of $\mathrm{GL}_{m_{i}}(\mathbb{A})$;

- $d_{i}$ is a positive integer such that $\sum_{i=1}^{t} m_{i} d_{i}$ is equal to $2 n+1$ (resp. $2 n$ );

- if $d_{i}$ is odd (resp. even), then $L\left(s, \tau_{i}, \mathrm{Sym}^{2}\right)$ has a pole at $s=1$;

- if $d_{i}$ is even (resp. odd), then $L\left(s, \tau_{i}, \wedge^{2}\right)$ has a pole at $s=1$;

- the central character $\omega_{i}$ of $\tau_{i}$ satisfies that $\omega_{1}^{d_{1}} \cdots \omega_{l}^{d_{l}}=\mathbf{1}$;

- if $i \neq j$ and $\tau_{i} \cong \tau_{j}$, then $d_{i} \neq d_{j}$.

Two $A$-parameters $\Psi=\boxplus_{i=1}^{t} \tau_{i}\left[d_{i}\right]$ and $\Psi^{\prime}=\boxplus_{i=1}^{t^{\prime}} \tau_{i}^{\prime}\left[d_{i}^{\prime}\right]$ are said to be equivalent if $t=t^{\prime}$ and there exists a permutation $\sigma \in \mathfrak{S}_{t}$ such that $d_{i}^{\prime}=d_{\sigma(i)}$ and $\tau_{i}^{\prime} \cong \tau_{\sigma(i)}$ for each $i$. We denote the set of equivalence classes of discrete global $A$-parameters for $\operatorname{Sp}_{n}(F)$ (resp. $\widetilde{\operatorname{Sp}}_{n}(F)$ ) by $\Psi_{2}\left(\operatorname{Sp}_{n}(F)\right.$ ) (resp. $\left.\Psi_{2}\left({\widetilde{\mathrm{Sp}_{n}}}_{n}(F)\right)\right)$. We call an $A$-parameter $\Psi=\boxplus_{i=1}^{t} \tau_{i}\left[d_{i}\right]$ tempered if $d_{i}=1$ for any $i$. In this case, we write $\Psi=\boxplus_{i=1}^{t} \tau_{i}$ for simplicity.

Let $\Psi=\boxplus_{i=1}^{t} \tau_{i}$ be a tempered $A$-parameter for $\operatorname{Sp}_{n}(F)$ (resp. $\widetilde{\mathrm{Sp}_{n}}(F)$ ). For each place $v$ of $F$, we denote the representation of $W D_{F_{v}}$ corresponding to $\tau_{i, v}$ by $\phi_{i, v}$, and put $\Psi_{v}=\oplus_{i=1}^{t} \phi_{i, v}$. By $[3$, Theorem 1.4.2], we have $\Psi_{v} \in \Phi\left(\operatorname{Sp}_{n}\left(F_{v}\right)\right)$ (resp. $\Psi_{v} \in \Phi\left(\widetilde{\operatorname{Sp}}_{n}\left(F_{v}\right)\right)$ ). We define a global $A$-packet $\Pi_{\Psi}$ by

$$
\Pi_{\Psi}=\left\{\pi=\otimes_{v}^{\prime} \pi_{v} \mid \pi_{v} \in \Pi_{\Psi_{v}}, \pi_{v} \text { is unramified for almost all } v\right\} .
$$

For a tempered $A$-parameter $\Psi=\boxplus_{i=1}^{t} \tau_{i}$, the global component group $A_{\Psi}$ of $\Psi$ is defined by

$$
A_{\Psi}=\bigoplus_{i=1}^{t}(\mathbb{Z} / 2 \mathbb{Z}) \alpha_{i}
$$


Namely, $A_{\Psi}$ is a free $\mathbb{Z} / 2 \mathbb{Z}$-module of rank $t$, and $\left\{\alpha_{1}, \ldots, \alpha_{t}\right\}$ is a basis of $A_{\Psi}$ with $\alpha_{i}$ associated to $\tau_{i}$. There exists a localization map $A_{\Psi} \ni \alpha \mapsto \alpha_{v} \in A_{\Psi_{v}}$, so that we have a diagonal map

$$
\Delta: A_{\Psi} \rightarrow \prod_{v} A_{\Psi_{v}} .
$$

Let $\mathcal{A}_{2}\left(\widetilde{\mathrm{Sp}_{n}}(\mathbb{A})\right)\left(\right.$ resp. $\mathcal{A}_{2}\left(\operatorname{Sp}_{n}(\mathbb{A})\right)$ ) be the set of square-integrable automorphic forms on $\widetilde{\mathrm{Sp}_{n}}(\mathbb{A})$ which are genuine (resp. not genuine). This is an $\widetilde{\operatorname{Sp}}_{n}\left(\mathbb{A}_{\text {fin }}\right) \times \prod_{v \mid \infty}\left(\mathfrak{s p} n(\mathbb{C}), \widetilde{K}_{\infty}\right)$-module. Arthur's multiplicity formula classifies $\mathcal{A}_{2}\left(\widetilde{\mathrm{Sp}_{n}}(\mathbb{A})\right)\left(\operatorname{resp} . \mathcal{A}_{2}\left(\operatorname{Sp}_{n}(\mathbb{A})\right)\right)$ in terms of $\Psi_{2}\left(\widetilde{\operatorname{Sp}}_{n}(F)\right)\left(\operatorname{resp} . \Psi_{2}\left(\operatorname{Sp}_{n}(F)\right)\right.$.

Theorem B.7 (Arthur's multiplicity formula ([3, Theorem 1.5.2], [14, Theorems 1.1, 1.3])). Let $G=\mathrm{Sp}_{n}$ or $G={\widetilde{\mathrm{Sp}_{n}}}_{\text {. }}$

(1) For each $\Psi \in \Psi_{2}(G(F))$, there exists an $\widetilde{\operatorname{Sp}}_{n}\left(\mathbb{A}_{\mathrm{fin}}\right) \times \prod_{v \mid \infty}\left(\mathfrak{s p},(\mathbb{C}), \widetilde{K}_{\infty}\right)$-submodule $\mathcal{A}_{2, \Psi}$ of $\mathcal{A}_{2}(G(\mathbb{A}))$ such that

$$
\mathcal{A}_{2}(G(\mathbb{A}))=\bigoplus_{\Psi \in \Psi_{2}(G(F))} \mathcal{A}_{2, \Psi}
$$

(2) Suppose that $\pi=\otimes_{v}^{\prime} \pi_{v}$ is an irreducible subrepresentation of $\mathcal{A}_{2, \Psi}$ with $\Psi=\boxplus_{i=1}^{t} \tau_{i}\left[d_{i}\right]$. Then for almost all $v<\infty$, the local factors $\pi_{v}$ of $\pi$ and $\tau_{i, v}$ of $\tau_{i}$ are unramified for any $i$. Moreover, if we denote the Satake parameter for $\tau_{i, v}$ by $\left\{c_{i, v, 1}, \ldots, c_{i, v, m_{i}}\right\}$, then the Satake parameter of $\pi_{v}$ is equal to

$$
\bigcup_{i=1}^{t} \bigcup_{j=1}^{m_{i}}\left\{c_{i, v, j} q^{-\frac{d_{i}-1}{2}}, c_{i, v, j} q^{-\frac{d_{i}-3}{2}}, \ldots, c_{i, v, j} q^{\frac{d_{i}-1}{2}}\right\}
$$

as multisets.

(3) When $\Psi=\boxplus_{i=1}^{t} \tau_{i}$ is a tempered A-parameter, $\mathcal{A}_{2, \Psi}$ is the multiplicity-free direct sum of representations $\pi=\otimes_{v}^{\prime} \pi_{v} \in \Pi_{\Psi}$ such that the character $\eta_{v}$ of $A_{\Psi_{v}}$ associated to $\pi_{v}$ satisfies the equation

$$
\left(\prod_{v} \eta_{v}\right) \circ \Delta\left(\alpha_{i}\right)= \begin{cases}1 & \text { if } G=\mathrm{Sp}_{n}, \\ \varepsilon\left(\tau_{i}\right) & \text { if } G={\widetilde{\mathrm{Sp}_{n}}}_{n} .\end{cases}
$$

Here, $\varepsilon\left(\tau_{i}\right)=\varepsilon\left(1 / 2, \tau_{i}, \psi\right) \in\{ \pm 1\}$ is the global root number attached to the symplectic representation $\tau_{i}$.

If a representation $\pi$ of $\widetilde{\operatorname{Sp}_{n}}(\mathbb{A})$ is contained in $\mathcal{A}_{2, \Psi}$, we say that $\pi$ has an $A$-parameter $\Psi$, and $\Psi$ is the $A$-parameter for $\pi$. We note that the following.

Lemma B.8. If $\Psi$ is a tempered A-parameter, and $\pi=\otimes_{v}^{\prime} \pi_{v}$ is an irreducible subrepresentation of $\mathcal{A}_{2, \Psi}$, then the local factors $\pi_{v}$ are almost tempered and unitary for all $v$.

Proof. Since any representation $\pi$ contained in $\mathcal{A}_{2, \Psi}$ is unitary, its local factors $\pi_{v}$ are unitary for all $v$. By the toward Ramanujan conjecture (see [30, (2.5) Corollary] and [45, Appendix]), we see that $\pi_{v}$ are almost tempered for all $v$.

Let $l=\left(l_{v}\right)_{v} \in \prod_{v \mid \infty} \mathbb{Z}$ with $l_{v}>0$ and $l_{v} \equiv l_{v^{\prime}} \bmod 2$ for any $v, v^{\prime} \mid \infty$. For $\Psi \in \Psi_{2}\left(\operatorname{Sp}_{n}(F)\right)$ if all $l_{v}$ are even, and for $\Psi \in \Psi_{2}\left(\widetilde{\operatorname{Sp}}_{n}(F)\right)$ if all $l_{v}$ are odd, we set

$$
\mathcal{S}_{l / 2, \Psi}=\mathcal{A}_{2, \Psi} \cap \mathcal{S}_{l / 2}\left(\operatorname{Sp}_{n}(F) \backslash \widetilde{\operatorname{Sp}}_{n}(\mathbb{A})\right) .
$$

Hence

$$
\mathcal{S}_{l / 2}\left(\operatorname{Sp}_{n}(F) \backslash \widetilde{\operatorname{Sp}}_{n}(\mathbb{A})\right) \cong \bigoplus_{\Psi \in \Psi_{2}(G(F))} \mathcal{S}_{l / 2, \Psi}
$$


By Proposition B.6, if $\Psi$ is tempered and $\mathcal{S}_{l / 2, \Psi}$ is nonzero, then $\Psi_{v}=\phi_{l_{v}}^{(n)}$ for each $v \mid \infty$. The subspace $\mathcal{S}_{l / 2, \Psi}$ is often zero.

Example B.9. Suppose that $n=1$. There is a unique non-tempered A-parameter $\mathbf{1}[3]$ for $\operatorname{Sp}_{1}(F)$, where $\mathbf{1}$ is the trivial representation of $\mathrm{GL}_{1}(\mathbb{A})$. The associated subspace $\mathcal{A}_{2, \mathbf{1}[3]}$ is the space of constant functions, i.e., $\mathcal{A}_{2, \mathbf{1}[3]}$ is the trivial $\mathrm{Sp}_{1}\left(\mathbb{A}_{\mathrm{fin}}\right) \times \prod_{v \mid \infty}\left(\mathfrak{s p}_{1}(\mathbb{C}), K_{\infty}\right)$-module. On the other hand, the non-tempered A-parameters for $\widetilde{\operatorname{Sp}}_{1}(F)$ are of the form $\chi_{\xi}[2]$. The associated space $\mathcal{A}_{2, \chi_{\xi}[2]}$ is isomorphic to the even Weil representation $\omega_{\psi_{\xi}}^{+}$as $\widetilde{\operatorname{Sp}}_{1}\left(\mathbb{A}_{\text {fin }}\right) \times \prod_{v \mid \infty}\left(\mathfrak{s p}_{1}(\mathbb{C}), \widetilde{K}_{\infty}\right)$-modules. Therefore, for $\Psi=\mathbf{1}[3]$ or $\Psi=\chi_{\xi}[2]$, we have $\mathcal{S}_{l / 2, \Psi}=0$ since the trivial representation and the even Weil representations are not cuspidal. In other words, if $n=1$ and $\mathcal{S}_{l / 2, \Psi} \neq 0$, then $\Psi$ is tempered.

To show Theorem 5.5, we need the following lemma.

Lemma B.10. Let $\tau=\otimes_{v}^{\prime} \tau_{v}$ be an irreducible unitary cuspidal automorphic representation of $\mathrm{GL}_{2}(\mathbb{A})$ satisfying the conditions (A1), (A2) and (A3) in §2.5. We denote the weight of $\otimes_{v \mid \infty} \tau_{v}$ by $\pm 2 k=\left( \pm 2 k_{v}\right)_{v}$ with $k_{v}>0$. Let $\Psi=\boxplus_{i=1}^{t} \tau_{i}$ be a tempered A-parameter for $\widetilde{\operatorname{Sp}}_{r}(F)$. Assume that for each infinite place $v \mid \infty$, the local factor $\Psi_{v}$ is equal to $\phi_{l_{v}}^{(r)}$ with $l_{v}=2 k_{v}+2 r+1$. Then $\Psi^{\prime}=\Psi \boxplus \tau \chi_{-1}^{r}$ is a tempered A-parameter for $\widetilde{\operatorname{Sp}}_{r+1}(F)$. Moreover, for any irreducible subrepresentation $\pi=\otimes_{v<\infty}^{\prime} \pi_{v}$ of $\mathcal{S}_{l / 2, \Psi}$, there exists an irreducible subrepresentation $\pi^{\prime}=\otimes_{v<\infty}^{\prime} \pi_{v}^{\prime}$ of $\mathcal{S}_{l / 2, \Psi^{\prime}}$ such that for each finite place $v<\infty$, the L-parameters $\left(\Psi_{v}, \eta_{v}\right)$ and $\left(\Psi_{v}^{\prime}, \eta_{v}^{\prime}\right)$ for $\pi$ and $\pi^{\prime}$, respectively, are related by $\eta_{v}^{\prime}=\eta_{v}$ under the canonical identification $A_{\Psi_{v}^{\prime}}=A_{\Psi_{v}}$.

Proof. For $v \mid \infty$, the local factor $\Psi_{v}=\phi_{l_{v}}^{(r)}$ does not contain $\rho_{2 k_{v}-1}$, which is the local factor of $\tau \chi_{-1}^{r}$ at $v$. Since the central character of $\tau \chi_{-1}^{r}$ is trivial, the exterior square $L$-function $L\left(s, \tau \chi_{-1}^{r}, \wedge^{2}\right)$ has a pole at $s=1$ by [33, Corollary 7.5]. Hence $\Psi^{\prime}=\Psi \boxplus \tau \chi_{-1}^{r}$ is a tempered $A$-parameter for $\widetilde{\mathrm{Sp}}_{r+1}(F)$.

Let $\pi=\otimes_{v<\infty}^{\prime} \pi_{v}$ be an irreducible subrepresentation of $\mathcal{S}_{l / 2, \Psi}$, and define $\pi^{\prime}=\otimes_{v<\infty}^{\prime} \pi_{v}^{\prime}$ so that the $L$-parameter for $\pi_{v}^{\prime}$ is equal to $\left(\Psi_{v}^{\prime}, \eta_{v}\right)$ for each $v<\infty$, where $\left(\Psi_{v}, \eta_{v}\right)$ is the $L$-parameter for $\pi_{v}$. To show that $\pi^{\prime}$ is a subrepresentation of $\mathcal{S}_{l / 2, \Psi^{\prime}}$, we use Arthur's multiplicity formula (Theorem B.7 (3)). Note that the $L$-parameters $\left(\phi_{l_{v} / 2}^{(r)}, \eta_{l_{v}}\right)$ and $\left(\phi_{l_{v} / 2}^{(r+1)}, \eta_{l_{v}}^{\prime}\right)$ for $\mathcal{D}_{l_{v} / 2}^{(r)}$ and $\mathcal{D}_{l_{v} / 2}^{(r+1)}$ are related by $\eta_{l_{v}}^{\prime} \mid A_{\phi_{l_{v} / 2}^{(r)}}=\eta_{l_{v}}$ and $\eta_{l_{v}}^{\prime}\left(a_{v}\right)=(-1)^{r}$, where $a_{v} \in A_{\phi_{l_{v} / 2}^{(r+1)}}$ is the element associated to $\rho_{2 k_{v}-1}$. Hence for the element $\alpha_{i} \in A_{\Psi} \subset A_{\Psi^{\prime}}$ associated to $\tau_{i}$, we have

$$
\left(\prod_{v<\infty} \eta_{v}^{\prime}\left(\alpha_{i, v}\right)\right)\left(\prod_{v \mid \infty} \eta_{l_{v}}^{\prime}\left(\alpha_{i, v}\right)\right)=\left(\prod_{v<\infty} \eta_{v}\left(\alpha_{i, v}\right)\right)\left(\prod_{v \mid \infty} \eta_{l_{v}}\left(\alpha_{i, v}\right)\right)=\varepsilon\left(\tau_{i}\right)
$$

and for the element $\alpha \in A_{\Psi^{\prime}}$ associated to $\tau \chi_{-1}^{r}$, we have

$$
\begin{aligned}
& \left(\prod_{v<\infty} \eta_{v}^{\prime}\left(\alpha_{v}\right)\right)\left(\prod_{v \mid \infty} \eta_{l_{v}}^{\prime}\left(\alpha_{v}\right)\right)=\left(\prod_{v \mid \infty}(-1)^{r}\right)=\left(\prod_{v \mid \infty} \chi_{-1, v}^{r}(-1)\right) \\
& =\left(\prod_{v<\infty}\left(\mu_{v} \chi_{-1, v}^{r}\right)(-1)\right)\left(\prod_{v \mid \infty}(-1)^{k_{v}}\right)=\varepsilon\left(\tau \chi_{-1}^{r}\right) .
\end{aligned}
$$

Here, we use the condition (A3) on $\tau$. By Arthur's multiplicity formula (Theorem B.7 (3)), we see that $\pi^{\prime}$ occurs in $\mathcal{S}_{l / 2, \Psi^{\prime}}$. 


\section{Appendix C. Gan-Gross-Prasad Conjectures}

The Gan-Gross-Prasad conjecture (the GGP conjecture) predicts a relation between the nonvanishing of the Fourier-Jacobi periods with the non-vanishing of the central values of the RankinSelberg $L$-functions. In this appendix, we review the statements of the GGP conjecture, its refined version and its local version. As an application of local Miyawaki liftings, we prove a new case of the local GGP conjecture in $\S$ C.3.

C.1. Global GGP conjectures. Let $F$ be a totally real number field, and $\psi$ be a non-trivial unitary character of $\mathbb{A} / F$. Recall that for $\xi \in F^{\times}$, the Weil representation $\omega_{\psi_{\xi}}$ of $\widetilde{J}_{n}(\mathbb{A})=\widetilde{\mathrm{Sp}}_{n}(\mathbb{A}) \ltimes$ $V_{n}(\mathbb{A})$ is realized on $\mathcal{S}\left(X_{n}(\mathbb{A})\right)$. For $\phi \in \mathcal{S}\left(X_{n}(\mathbb{A})\right)$, we define the theta function by

$$
\Theta_{\psi_{\xi}}^{\phi}\left(v g^{\prime}\right)=\sum_{x \in X_{n}(F)} \omega_{\psi_{\xi}}\left(v g^{\prime}\right) \phi(x)
$$

for $v \in V_{n}(\mathbb{A})$ and $g^{\prime} \in \widetilde{\mathrm{Sp}_{n}}(\mathbb{A})$. This is a genuine automorphic form on $\widetilde{J}_{n}(\mathbb{A})$.

Suppose that $n^{\prime}=n$ or $n^{\prime}=n-1$. Let $\varphi$ and $\varphi^{\prime}$ be cusp forms on $\widetilde{\operatorname{Sp}}_{n}(\mathbb{A})$ and $\widetilde{\operatorname{Sp}_{n^{\prime}}}(\mathbb{A})$, respectively. Assume that exactly one of $\varphi$ or $\varphi^{\prime}$ is genuine. Then for $\phi \in \mathcal{S}\left(X_{n^{\prime}}(\mathbb{A})\right)$, we define the Fourier-Jacobi period $\mathcal{P}_{n, n^{\prime}, \psi_{\xi}}\left(\varphi, \varphi^{\prime}, \phi\right)$ by

$$
\begin{aligned}
\mathcal{P}_{n, n, \psi_{\xi}}\left(\varphi, \varphi^{\prime}, \phi\right) & =\int_{\operatorname{Sp}_{n}(F) \backslash \operatorname{Sp}_{n}(\mathbb{A})} \varphi(g, \zeta) \varphi^{\prime}(g, \zeta) \overline{\Theta_{\psi_{\xi}}^{\phi}(g, \zeta)} d g \\
\mathcal{P}_{n, n-1, \psi_{\xi}}\left(\varphi, \varphi^{\prime}, \phi\right) & =\int_{V_{n-1}(F) \backslash V_{n-1}(\mathbb{A})} \int_{\operatorname{Sp}_{n-1}(F) \backslash \operatorname{Sp}_{n-1}(\mathbb{A})} \varphi(v(g, \zeta)) \varphi^{\prime}(g, \zeta) \overline{\Theta_{\psi_{\xi}}^{\phi}(v(g, \zeta))} d g d v .
\end{aligned}
$$

The GGP conjecture is stated as follows:

Conjecture C.1 (Gan-Gross-Prasad conjecture [11, Conjecture 24.1]). Suppose that $n^{\prime}=n$ or $n^{\prime}=n-1$. Let $\pi=\otimes_{v}^{\prime} \pi_{v}$ and $\pi^{\prime}=\otimes_{v}^{\prime} \pi_{v}^{\prime}$ be irreducible cuspidal automorphic representations of $\widetilde{\operatorname{Sp}_{n}}(\mathbb{A})$ and $\widetilde{\mathrm{Sp}_{n^{\prime}}}(\mathbb{A})$, respectively. Assume that exactly one of $\pi$ or $\pi^{\prime}$ is genuine, and that the A-parameters for $\pi$ and $\pi^{\prime}$ are tempered. Then the following are equivalent:

(1) The Fourier-Jacobi period $\mathcal{P}_{n, n^{\prime}, \psi_{\xi}}$ is not identically zero on $\pi \times \pi^{\prime} \times \mathcal{S}\left(X_{n^{\prime}}(\mathbb{A})\right)$.

(2) The central value $L\left(1 / 2, \pi \times \pi^{\prime} \times \chi_{\xi}\right)$ is nonzero and the local Hom-space

$$
\operatorname{Hom}_{\mathrm{Sp}_{n^{\prime}}\left(F_{v}\right)}\left(\pi_{v} \otimes \pi_{v}^{\prime} \otimes \overline{\omega_{\psi_{\xi, v}}}, \mathbb{C}\right)
$$

is nonzero for any place $v$ of $F$.

For the case where $n^{\prime}=n$, Yamana [58, Theorem 1.1] proved that (1) implies (2). See also [58, Remark 6.6]. Conjecture C.1 implies Conjecture 5.2 (1) since the $A$-parameter for $\overline{\pi^{\prime}}$ is given by

$$
\begin{cases}\Psi^{\prime}=\boxplus_{j=1}^{t^{\prime}} \tau_{j}^{\prime} & \text { if } \pi^{\prime} \text { is not genuine, } \\ \Psi^{\prime} \otimes \chi_{-1}=\boxplus_{j=1}^{t^{\prime}}\left(\tau_{j}^{\prime} \otimes \chi_{-1}\right) & \text { if } \pi^{\prime} \text { is genuine, }\end{cases}
$$

where $\Psi^{\prime}=\boxplus_{j=1}^{t^{\prime}} \tau_{j}^{\prime}$ is the $A$-parameter for $\pi^{\prime}$.

To obtain Conjecture 5.2 (2), we need a refined version of Conjecture C.1. To state this, we define a local period integral. For each place $v$, we fix a Haar measure on $\operatorname{Sp}_{n^{\prime}}\left(F_{v}\right)$ such that $\operatorname{vol}\left(\operatorname{Sp}_{n^{\prime}}\left(\mathfrak{o}_{v}\right) ; d g_{v}^{\prime}\right)=1$ for almost all $v$, and that $d g^{\prime}=\prod_{v} d g_{v}^{\prime}$ is the Tamagawa measure on $\operatorname{Sp}_{n^{\prime}}(\mathbb{A})$. Fix an $\operatorname{Sp}_{n}\left(F_{v}\right)$-invariant inner product $\langle,\rangle_{\pi_{v}}$ of $\pi_{v} \times \pi_{v}$ such that

$$
\int_{\operatorname{Sp}_{n}(F) \backslash \operatorname{Sp}_{n}(\mathbb{A})} \varphi_{1}(g) \overline{\varphi_{2}(g)} d g=\prod_{v}\left\langle\varphi_{1, v}, \varphi_{2, v}\right\rangle_{\pi_{v}}
$$


for $\varphi_{1}=\otimes_{v} \varphi_{1, v}, \varphi_{2}=\otimes_{v} \varphi_{2, v} \in \pi$. Similarly, fix an $\operatorname{Sp}_{n^{\prime}}\left(F_{v}\right)$-invariant inner product $\langle,\rangle_{\pi_{v}^{\prime}}$ of $\pi_{v}^{\prime} \times \pi_{v}^{\prime}$. Recall that $\omega_{\psi_{\xi, v}}$ has a $\widetilde{J}_{n^{\prime}}\left(F_{v}\right)$-invariant pairing

$$
\left(\phi_{1, v}, \phi_{2, v}\right)_{v}=\int_{X_{n^{\prime}}\left(F_{v}\right)} \phi_{1, v}\left(x_{v}\right) \overline{\phi_{2, v}\left(x_{v}\right)} d x_{v}
$$

which satisfies that $\left(\phi_{1}, \phi_{2}\right)=\prod_{v}\left(\phi_{1, v}, \phi_{2, v}\right)_{v}$ for $\phi_{1}=\otimes_{v} \phi_{1, v}, \phi_{2}=\otimes_{v} \phi_{2, v} \in \mathcal{S}\left(X_{n^{\prime}}(\mathbb{A})\right)$. For $\varphi_{v} \in \pi_{v}, \varphi_{v}^{\prime} \in \pi_{v}^{\prime}$ and $\phi_{v} \in \mathcal{S}\left(X_{n^{\prime}}\left(F_{v}\right)\right)$, we define a local period integral by

$$
\begin{aligned}
& \alpha_{v}\left(\varphi_{v}, \varphi_{v}^{\prime}, \phi_{v}\right) \\
& = \begin{cases}\int_{\operatorname{Sp}_{n}\left(F_{v}\right)}\left\langle\pi_{v}(g) \varphi_{v}, \varphi_{v}\right\rangle_{\pi_{v}}\left\langle\pi_{v}^{\prime}(g) \varphi_{v}^{\prime}, \varphi_{v}^{\prime}\right\rangle_{\pi_{v}^{\prime}} \overline{\left(\omega_{\psi_{\xi, v}}(g) \phi_{v}, \phi_{v}\right)_{v}} d g & \text { if } n^{\prime}=n, \\
\int_{V_{n-1}\left(F_{v}\right)} \int_{\operatorname{Sp}_{n-1}\left(F_{v}\right)}\left\langle\pi_{v}(u g) \varphi_{v}, \varphi_{v}\right\rangle_{\pi_{v}}\left\langle\pi_{v}^{\prime}(g) \varphi_{v}^{\prime}, \varphi_{v}^{\prime}\right\rangle_{\pi_{v}^{\prime}} \overline{\left(\omega_{\psi_{\xi, v}}(u g) \phi_{v}, \phi_{v}\right)_{v}} d g d u & \text { if } n^{\prime}=n-1 .\end{cases}
\end{aligned}
$$

This integral is absolutely convergent by [56, Proposition 2.2.1].

The refined version of the GGP conjecture is stated as follows:

Conjecture C.2 (Xue [56, Conjecture 2.3.1]). Let $\pi=\otimes_{v}^{\prime} \pi_{v}$ and $\pi^{\prime}=\otimes_{v}^{\prime} \pi_{v}^{\prime}$ be irreducible cuspidal automorphic representations of $\widetilde{\mathrm{Sp}}_{n}(\mathbb{A})$ and $\widetilde{\mathrm{Sp}}_{n^{\prime}}(\mathbb{A})$ with tempered $A$-parameters $\Psi$ and $\Psi^{\prime}$, respectively. Assume that exactly one of $\pi$ or $\pi^{\prime}$ is genuine. Fix a sufficiently large finite set $S$ of places of $F$ containing all "bad" places.

(1) For each place $v$, the local Hom-space $\operatorname{Hom}_{\mathrm{Sp}_{n^{\prime}}\left(F_{v}\right)}\left(\pi_{v} \otimes \pi_{v}^{\prime} \otimes \overline{\omega_{\psi_{\xi, v}}}, \mathbb{C}\right)$ is nonzero if and only if $\alpha_{v}\left(\varphi_{v}, \varphi_{v}^{\prime}, \phi_{v}\right) \neq 0$ for some $\varphi_{v} \in \pi_{v}, \varphi_{v}^{\prime} \in \pi_{v}^{\prime}$ and $\phi_{v} \in \omega_{\psi_{\xi, v}}$.

(2) Let $\varphi=\otimes_{v} \varphi_{v} \in \pi, \varphi^{\prime}=\otimes_{v} \varphi_{v}^{\prime} \in \pi^{\prime}$, and $\phi=\otimes_{v} \phi_{v} \in \mathcal{S}\left(X_{n^{\prime}}(\mathbb{A})\right)$ be factorizable elements. Then there exists a constant $\Delta^{S}$, which is an explicit product of partial zeta values, such that

$$
\begin{aligned}
& \left|\mathcal{P}_{n, n^{\prime}, \psi_{\xi}}\left(\varphi, \varphi^{\prime}, \phi\right)\right|^{2} \\
& =\left.\frac{2 \Delta^{S}}{\left|A_{\Psi}\right|\left|A_{\Psi^{\prime}}\right|} \frac{L^{S}\left(s, \pi \times \pi^{\prime} \times \chi_{\xi}\right)}{L^{S}\left(s+\frac{1}{2}, \pi, \mathrm{Ad}\right) L^{S}\left(s+\frac{1}{2}, \pi, \mathrm{Ad}\right)}\right|_{s=\frac{1}{2}} \times \prod_{v \in S} \alpha_{v}\left(\varphi_{v}, \varphi_{v}^{\prime}, \phi_{v}\right) .
\end{aligned}
$$

Here, $L^{S}\left(s, \pi \times \pi^{\prime} \times \chi_{\xi}\right)$ is the partial Rankin-Selberg L-function, and $L^{S}(s, \pi, \mathrm{Ad})$ and $L^{S}\left(s, \pi^{\prime}, \mathrm{Ad}\right)$ are the partial adjoint $L$-functions of $\pi$ and $\pi^{\prime}$, respectively.

Conjecture C.2 together with the following lemma implies Conjecture 5.2 (2).

Lemma C.3. Assume that $n^{\prime}=n$. Let $v$ be a real place of $F$, and $\xi$ be a positive real number (in $\left.F_{v}\right)$. Then for nonzero lowest weight vectors $\varphi_{l / 2} \in \mathcal{D}_{l / 2}^{(n)}, \varphi_{(l-1) / 2} \in \mathcal{D}_{(l-1) / 2}^{(n)}$, and $\phi_{\xi}^{0} \in \omega_{\psi_{\xi, v}}$, we have $\alpha_{v}\left(\varphi_{l / 2}, \overline{\varphi_{(l-1) / 2}}, \phi_{\xi}^{0}\right) \neq 0$.

Proof. Set $\pi_{v}=\mathcal{D}_{l / 2}^{(n)}$ and $\pi_{v}^{\prime}=\mathcal{D}_{(l-1) / 2}^{(n)}$. Note that the subspace of $\mathcal{D}_{(l-1) / 2}^{(n)} \otimes \omega_{\psi_{\xi, v}}$ on which $\widetilde{K}_{\infty}$ acts by $\operatorname{det}^{l / 2}$ is one dimension, and is spanned by $\varphi_{(l-1) / 2} \otimes \phi_{\xi}^{0}$. Since $\operatorname{Hom}_{\widetilde{S p}_{n}(\mathbb{R})}\left(\mathcal{D}_{l / 2}^{(n)}, \mathcal{D}_{(l-1) / 2}^{(n)} \otimes \omega_{\psi_{\xi, v}}\right) \neq$ 0 , we may regard $\varphi_{(l-1) / 2} \otimes \phi_{\xi}^{0}$ as a lowest weight vector of $\mathcal{D}_{l / 2}^{(n)} \subset \mathcal{D}_{(l-1) / 2}^{(n)} \otimes \omega_{\psi_{\xi, v}}$. Hence we can regard

$$
\left\langle\pi_{v}^{\prime}(g) \varphi_{(l-1) / 2}, \varphi_{(l-1) / 2}\right\rangle_{\pi_{v}^{\prime}}\left(\omega_{\psi_{\xi, v}}(g) \phi_{\xi}^{0}, \phi_{\xi}^{0}\right)_{v}
$$

as a (nonzero) matrix coefficient of $\mathcal{D}_{l / 2}^{(n)}$ on which the left-right translation of $\widetilde{K}_{\infty} \times \widetilde{K}_{\infty}$ is equal to $\operatorname{det}^{l / 2} \otimes \operatorname{det}^{l / 2}$. Since such a matrix coefficient is a scalar multiple of $\left\langle\pi_{v}(g) \varphi_{l / 2}, \varphi_{l / 2}\right\rangle_{\pi_{v}}$, we have $\alpha_{v}\left(\varphi_{l / 2}, \overline{\varphi_{(l-1) / 2}}, \phi_{\xi}^{0}\right) \neq 0$. 
C.2. Local GGP conjecture. In this subsection, we let $F$ be a non-archimedean local field of characteristic zero. Fix a non-trivial additive character $\psi$ of $F$ and an element $\xi \in F^{\times}$. Let $\pi_{1}$ and $\pi_{2}$ be irreducible representations of $\widetilde{\mathrm{Sp}}_{r}(F)$ and $\widetilde{\mathrm{Sp}}_{r-1}(F)$, respectively. Assume that exactly one of them is genuine. We set

$$
d_{r, r-1, \xi}\left(\pi_{1}, \pi_{2}\right)=\operatorname{dim} \operatorname{Hom}_{J_{r-1}(F)}\left(\pi_{1} \otimes \pi_{2} \otimes \overline{\omega_{\psi_{\xi}}^{(r-1)}}, \mathbb{C}\right) .
$$

Similarly, let $\pi_{1}^{\prime}$ and $\pi_{2}^{\prime}$ be irreducible representations of $\widetilde{\operatorname{Sp}_{n}}(F)$. Assume that exactly one of them is genuine. We set

$$
d_{n, n, \xi}\left(\pi_{1}^{\prime}, \pi_{2}^{\prime}\right)=\operatorname{dim} \operatorname{Hom}_{\operatorname{Sp}_{n}(F)}\left(\pi_{1}^{\prime} \otimes \pi_{2}^{\prime} \otimes \overline{\omega_{\psi_{\xi}}^{(n)}}, \mathbb{C}\right) .
$$

The multiplicity one theorem proven by Sun [48] and Gan-Gross-Prasad [11, Corollary 16.2] asserts that

for any $\pi_{1}, \pi_{2}, \pi_{1}^{\prime}, \pi_{2}^{\prime}$.

$$
d_{r, r-1, \xi}\left(\pi_{1}, \pi_{2}\right) \leq 1, \quad d_{n, n, \xi}\left(\pi_{1}^{\prime}, \pi_{2}^{\prime}\right) \leq 1
$$

For a symplectic representation $\phi$ of $W D_{F}$, we denote the root number attached to $\phi$ by $\varepsilon(\phi)=$ $\varepsilon(1 / 2, \phi, \psi)$. This value does not depend on $\psi$, and is in $\{ \pm 1\}$. The local GGP conjecture for the symplectic-metaplectic case (proven by the author [5]) gives complete descriptions of $d_{r, r-1, \xi}\left(\pi_{1}, \pi_{2}\right)$ and $d_{n, n, \xi}\left(\pi_{1}^{\prime}, \pi_{2}^{\prime}\right)$ in terms of internal structures of $L$-packets when all $\pi_{1}, \pi_{2}, \pi_{1}^{\prime}, \pi_{2}^{\prime}$ are almost tempered.

Theorem C.4 ([5, Theorem 1.3, Corollary 1.4], [11, Proposition 18.1]). Assume that all $\pi_{1}, \pi_{2}, \pi_{1}^{\prime}, \pi_{2}^{\prime}$ are almost tempered. Let $\left(\phi_{1}, \eta_{1}\right),\left(\phi_{2}, \eta_{2}\right),\left(\phi_{1}^{\prime}, \eta_{1}^{\prime}\right),\left(\phi_{2}^{\prime}, \eta_{2}^{\prime}\right)$ be the L-parameters for $\pi_{1}, \pi_{2}, \pi_{1}^{\prime}, \pi_{2}^{\prime}$, respectively. Then $d_{r, r-1, \xi}\left(\pi_{1}, \pi_{2}\right) \neq 0$ if and only if

$$
\left\{\begin{array}{l}
\eta_{1}(a)=\varepsilon\left(\phi_{1}^{a} \otimes \phi_{2} \otimes \chi_{\xi}\right) \varepsilon\left(\phi_{1} \otimes \phi_{2} \otimes \chi_{\xi}\right)^{\operatorname{det}(a)} \operatorname{det}\left(\phi_{1}^{a}\right)\left((-1)^{\frac{1}{2} \operatorname{dim}\left(\phi_{2}\right)} \xi\right), \\
\eta_{2}(b)=\varepsilon\left(\phi_{1} \otimes \phi_{2}^{b} \otimes \chi_{\xi}\right) \varepsilon\left(\phi_{2}^{b}\right) \chi_{\xi}(-1)^{\frac{1}{2} \operatorname{dim}\left(\phi_{2}^{b}\right)}
\end{array}\right.
$$

for $a \in A_{\phi_{1}}$ and $b \in A_{\phi_{2}}$, and $d_{n, n, \xi}\left(\pi_{1}^{\prime}, \pi_{2}^{\prime}\right) \neq 0$ if and only if

$$
\left\{\begin{array}{l}
\eta_{1}^{\prime}(a)=\varepsilon\left(\phi_{1}^{\prime a} \otimes \phi_{2}^{\prime} \otimes \chi_{\xi}\right) \varepsilon\left(\phi_{1}^{\prime} \otimes \phi_{2}^{\prime} \otimes \chi_{\xi}\right)^{\operatorname{det}(a)} \operatorname{det}\left(\phi_{1}^{\prime a}\right)\left((-1)^{\frac{1}{2} \operatorname{dim}\left(\phi_{2}^{\prime}\right)} \xi\right), \\
\eta_{2}^{\prime}(b)=\varepsilon\left(\phi_{1}^{\prime} \otimes \phi_{2}^{\prime b} \otimes \chi_{\xi}\right) \varepsilon\left(\phi_{2}^{\prime b}\right) \chi_{\xi}(-1)^{\frac{1}{2} \operatorname{dim}\left({\phi^{\prime}}_{2}^{b}\right)}
\end{array}\right.
$$

for $a \in A_{\phi_{1}^{\prime}}$ and $b \in A_{\phi_{2}^{\prime}}$.

C.3. Application of local Miyawaki liftings. As in Proposition 3.10, local Miyawaki liftings satisfy a seesaw identity. Using this, one can describe $d_{n, n, \xi}\left(\pi_{1}^{\prime}, \pi_{2}^{\prime}\right)$ for a new case. Recall that the unique irreducible algebraic representation of $\mathrm{SL}_{2}(\mathbb{C})$ of dimension $d$ is denoted by $S_{d}$.

Theorem C.5. Let $\pi_{1}$ and $\pi_{2}$ be irreducible almost tempered unitary representations of $\widetilde{\operatorname{Sp}}_{r}(F)$ and $\widetilde{\operatorname{Sp}}_{r-1}(F)$, on which $\{ \pm 1\}$ acts by $( \pm 1)^{n+r}$ and $( \pm 1)^{n+r-1}$, respectively. Fix a unitary character $\mu$ of $F^{\times}$.

(1) Assume one of the following conditions:

- The L-parameter $\phi_{1}$ for $\pi_{1}$ does not contain $\mu^{ \pm 1} S_{d}$ for any $d \geq n-r$ with $d \equiv n-$ $r \bmod 2$;

- $n=r$ or $n=r+1$.

Then we have

$$
d_{n, n, \xi}\left(\left(\mu \chi_{-1}^{n+r-1} \circ \operatorname{det}_{n-r}\right) \rtimes \pi_{1},\left(\mu \chi_{\xi} \circ \operatorname{det}_{n-r+1}\right) \rtimes \pi_{2}\right)=d_{r, r-1, \xi}\left(\pi_{1}, \pi_{2}\right) .
$$

In particular, $d_{n, n, \xi}\left(\left(\mu \chi_{-1}^{n+r-1} \circ \operatorname{det}_{n-r}\right) \rtimes \pi_{1},\left(\mu \chi_{\xi} \circ \operatorname{det}_{n-r+1}\right) \rtimes \pi_{2}\right)$ can be described in terms of internal structures of the L-packets for $\pi_{1}$ and $\pi_{2}$. 
(2) Set $\mu^{\prime}=\mu \chi_{-1}^{[(n+r-1) / 2]}$ and $\tau^{\prime}=\mu^{\prime} \times \mu^{\prime-1}$. If an irreducible representation $\pi_{1}^{\prime}$ of $\widetilde{\operatorname{Sp}}_{n}(F)$ satisfies that $d_{n, n, \xi}\left(\pi_{1}^{\prime},\left(\mu \chi_{\xi} \circ \operatorname{det}_{n-r+1}\right) \rtimes \pi_{2}\right) \neq 0$, then $\mathcal{M}_{\psi, \tau^{\prime}}^{(r)}\left(\pi_{1}^{\prime}\right) \neq 0$.

Proof. Note that $\mu \chi_{-1}^{n+r-1}=\mu^{\prime} \chi_{-1}^{[(n+r) / 2]}$. By Theorem 3.1 (2), (4) and the seesaw identity (Proposition 3.10), we have

$$
\begin{aligned}
& d_{n, n, \xi}\left(\left(\mu \chi_{-1}^{n+r-1} \circ \operatorname{det}_{n-r}\right) \rtimes \pi_{1},\left(\mu \chi_{\xi} \circ \operatorname{det}_{n-r+1}\right) \rtimes \pi_{2}\right) \neq 0 \\
& \Longleftrightarrow d_{n, n, \xi}\left(\mathcal{M}_{\psi, \tau^{\prime}}^{(n)}\left(\pi_{1}\right), \mathcal{M}_{\psi, \tau^{\prime} \chi_{\xi}}^{(n)}\left(\pi_{2}\right)\right) \neq 0 \\
& \Longleftrightarrow \operatorname{Hom}_{\widetilde{S p}_{n}(F)}\left(\mathcal{M}_{\psi, \tau^{\prime} \chi_{\xi}}^{(n)}\left(\pi_{2}^{\vee}\right) \otimes \omega_{\psi_{\xi}}^{(n)}, \mathcal{M}_{\psi, \tau^{\prime}}^{(n)}\left(\pi_{1}\right)\right) \neq 0 \\
& \Longleftrightarrow \operatorname{Hom}_{\widetilde{J}_{r-1}}\left(\mathcal{M}_{\psi, \tau^{\prime}}^{(r)}\left(\mathcal{M}_{\psi, \tau^{\prime}}^{(n)}\left(\pi_{1}\right)\right), \pi_{2}^{\vee} \otimes \omega_{\psi_{\xi}}^{(r-1)}\right) \neq 0 \\
& \Longleftrightarrow \operatorname{Hom}_{\widetilde{J}_{r-1}}\left(\pi_{1}, \pi_{2}^{\vee} \otimes \omega_{\psi_{\xi}}^{(r-1)}\right) \neq 0 \\
& \Longleftrightarrow d_{r, r-1, \xi}\left(\pi_{1}, \pi_{2}\right) \neq 0 .
\end{aligned}
$$

Hence we obtain (1).

When $d_{n, n, \xi}\left(\pi_{1}^{\prime},\left(\mu \chi_{\xi} \circ \operatorname{det}_{n-r+1}\right) \rtimes \pi_{2}\right) \neq 0$, the seesaw identity implies that

$$
\operatorname{Hom}_{\widetilde{J}_{r-1}}\left(\mathcal{M}_{\psi, \tau^{\prime}}^{(r)}\left(\pi_{1}^{\prime}\right), \pi_{2}^{\vee} \otimes \omega_{\psi}^{(r-1)}\right) \neq 0 .
$$

In particular, $\mathcal{M}_{\psi, \tau^{\prime}}^{(r)}\left(\pi_{1}^{\prime}\right) \neq 0$. Hence we have $(2)$.

\section{REFERENCES}

[1] J. Adams and D. Barbasch, Genuine representations of the metaplectic group, Compositio Math. 113 (1998), no. 1, 23-66.

[2] H. Aoki, Estimating Siegel modular forms of genus 2 using Jacobi forms, J. Math. Kyoto Univ. 40 (2000), no. $3,581-588$.

[3] J. Arthur, The endoscopic classification of representations. Orthogonal and symplectic groups, American Mathematical Society Colloquium Publications, 61. American Mathematical Society, Providence, RI, 2013. xviii+590 pp.

[4] M. Asgari and R. Schmidt, Siegel modular forms and representations, Manuscripta Math. 104 (2001), no. $2,173-200$.

[5] H. Atobe, The local theta correspondence and the local Gan-Gross-Prasad conjecture for the symplecticmetaplectic case, Math. Ann. 371 (2018), no. 1-2, 225-295.

[6] H. Atobe, On the non-vanishing of theta liftings of tempered representations of $\mathrm{U}(p, q)$, arXiv:1610.07794v1.

[7] H. Atobe and W. T. Gan, Local theta correspondence of tempered representations and Langlands parameters, Invent. Math. 210 (2017), no. 2, 341-415.

[8] D. Blasius, Hilbert modular forms and the Ramanujan conjecture, Noncommutative geometry and number theory, 35-56, Aspects Math., E37, Friedr. Vieweg, Wiesbaden, 2006.

[9] C. Bushnell, Representations of reductive p-adic groups: localization of Hecke algebras and applications, J. London Math. Soc. (2) 63 (2001), no. 2, 364-386.

[10] A. Caraiani, Local-global compatibility and the action of monodromy on nearby cycles, Duke Math. J. 161 (2012), no. 12, 2311-2413.

[11] W. T. Gan, B. H. Gross and D. Prasad, Symplectic local root numbers, central critical L-values, and restriction problems in the representation theory of classical groups, Astérisque. No. 346 (2012), 1-109.

[12] W. T. Gan and N. Gurevich, Restrictions of Saito-Kurokawa representations. With an appendix by Gordan Savin, Contemp. Math., 488, Israel Math. Conf. Proc., Automorphic forms and L-functions I. Global aspects, 95-124, Amer. Math. Soc., Providence, RI, 2009.

[13] W. T. Gan and A. Ichino, The Gross-Prasad conjecture and local theta correspondence, Invent. Math. 206 (2016), no. 3, 705-799. 
[14] W. T. Gan and A. Ichino, The Shimura-Waldspurger correspondence for $\mathrm{Mp}_{2 n}$, Ann. of Math. (2) 188 (2018), no. 3, 965-1016.

[15] W. T. Gan and G. Savin, Representations of metaplectic groups I: epsilon dichotomy and local Langlands correspondence, Compos. Math. 148 (2012), no. 6, 1655-1694.

[16] W. T. Gan and S. Takeda, On the Howe duality conjecture in classical theta correspondence, Advances in the theory of automorphic forms and their L-functions, 105-117, Contemp. Math., 664, Amer. Math. Soc., Providence, RI, 2016.

[17] P. Garrett and B. Heim, Hecke duality of Ikeda lifts, J. Number Theory 146 (2015), 171-186.

[18] D. Ginzburg, S. Rallis and D. Soudry, On explicit lifts of cusp forms from GL $_{m}$ to classical groups, Ann. of Math. (2) 150 (1999), no. 3, 807-866.

[19] D. Ginzburg, S. Rallis and D. Soudry, On Fourier coefficients of automorphic forms of symplectic groups, Manuscripta Math. 111 (2003), no. 1, 1-16.

[20] M. Hanzer and G. Muić, Parabolic induction and Jacquet functors for metaplectic groups, J. Algebra 323 (2010), no. 1, 241-260.

[21] M. Hanzer and G. Savin, Eisenstein series arising from Jordan algebras, Canadian Journal of Mathematics, http://dx.doi.org/10.4153/CJM-2018-033-2.

[22] S. Hayashida, Lifting from two elliptic modular forms to Siegel modular forms of half-integral weight of even degree, Doc. Math. 21 (2016), 125-196.

[23] V. Heiermann, A note on standard modules and Vogan L-packets, Manuscripta Math. 150 (2016), no. 3-4, $571-583$.

[24] T. Ibukiyama, C. Poor, and D. S. Yuen, Jacobi forms that characterize paramodular forms, Abh. Math. Semin. Univ. Hambg. 83 (2013), no. 1, 111-128.

[25] A. Ichino, Pullbacks of Saito-Kurokawa lifts, Invent. Math. 162 (2005), no. 3, 551-647.

[26] T. Ikeda, On the theory of Jacobi forms and Fourier-Jacobi coefficients of Eisenstein series, J. Math. Kyoto Univ. 34 (1994), no. 3, 615-636.

[27] T. Ikeda, On the lifting of elliptic cusp forms to Siegel cusp forms of degree 2n, Ann. of Math. (2) 154 (2001), no. 3, 641-681.

[28] T. Ikeda, Pullback of the lifting of elliptic cusp forms and Miyawaki's conjecture, Duke Math. J. 131 (2006), no. 3, 469-497.

[29] T. Ikeda and S. Yamana, On the lifting of Hilbert cusp forms to Hilbert-Siegel cusp forms, Ann. Sci. Ec. Norm. Sup. (4), to appear.

[30] H. Jacquet and J. A. Shalika, On Euler products and the classification of automorphic representations. I, Amer. J. Math. 103 (1981), no. 3, 499-558.

[31] C. Jantzen, Degenerate principal series for symplectic and odd-orthogonal groups, Mem. Amer. Math. Soc. 124 (1996), no. 590, viii+100 pp.

[32] D. Jiang and L. Zhang, Arthur parameters and cuspidal automorphic modules of classical groups, arXiv:1508.03205v3.

[33] P. K. Kewat and R. Raghunathan, On the local and global exterior square L-functions of $\mathrm{GL}_{n}$, Math. Res. Lett. 19 (2012), no. 4, 785-804.

[34] T. Konno, A note on the Langlands classification and irreducibility of induced representations of p-adic groups, Kyushu J. Math. 57 (2003), no. 2, 383-409.

[35] S. S. Kudla and S. Rallis, Ramified degenerate principal series representations for $\operatorname{Sp}(n)$, Israel J. Math. 78 (1992), no. 2-3, 209-256.

[36] S. S. Kudla and S. Rallis, On first occurrence in the local theta correspondence, Automorphic representations, L-functions and applications: progress and prospects, 273-308, Ohio State Univ. Math. Res. Inst. Publ., 11, de Gruyter, Berlin, 2005.

[37] R. P. Langlands, On the classification of irreducible representations of real algebraic groups, Representation theory and harmonic analysis on semisimple Lie groups, 101-170, Math. Surveys Monogr., 31, Amer. Math. Soc., Providence, RI, 1989.

[38] E. Lapid and S. Rallis, On the local factors of representations of classical groups, Automorphic representations, L-functions and applications: progress and prospects, 309-359, Ohio State Univ. Math. Res. Inst. Publ., 11, de Gruyter, Berlin, 2005.

[39] I. Miyawaki, Numerical examples of Siegel cusp forms of degree 3 and their zeta-functions, Mem. Fac. Sci. Kyushu Univ. Ser. A 46 (1992), no. 2, 307-339.

[40] C. Møglin, M.-F. Vignéras and J.-L. Waldspurger, Correspondances de Howe sur un corps p-adique, Lecture Notes in Mathematics, 1291. Springer-Verlag, Berlin, 1987. viii+163 pp. 
[41] C. Møglin and J.-L. Waldspurger, Le spectre résiduel de GL(n), Ann. Sci. École Norm. Sup. (4) 22 (1989), no. 4, 605-674.

[42] P. D. Nelson and A. Venkatesh, The orbit method and analysis of automorphic forms, arXiv:1805.07750v2.

[43] I. I. Piatetski-Shapiro and S. Rallis, L-functions for the classical groups, Explicit constructions of automorphic L-functions, Lecture Notes in Mathematics 1254, Springer-Verlag, Berlin, 1987, pp. 1-52.

[44] Y. Qiu, Periods of Saito-Kurokawa representations, Int. Math. Res. Not. IMRN 2014 (2014), no. 24, $6698-6755$.

[45] Z. Rudnick and P. Sarnak, Zeros of principal L-functions and random matrix theory, Duke Math. J. 81 (1996), no. 2, 269-322.

[46] W. Schmid, On the characters of the discrete series. The Hermitian symmetric case, Invent. Math. 30 (1975), no. 1, 47-144.

[47] W. Schmid, Two character identities for semisimple Lie groups, Non-commutative harmonic analysis (Actes Colloq., Marseille-Luminy, 1976), pp. 196-225. Lecture Notes in Math., Vol. 587, Springer, Berlin, 1977.

[48] B. Sun, Multiplicity one theorems for Fourier-Jacobi models, Amer. J. Math. 134 (2012), no. 6, 16551678.

[49] B. Sun, On representations of real Jacobi groups, Sci. China Math. 55 (2012), no. 3, 541-555.

[50] W. J. Sweet, Jr., Functional equations of p-adic zeta integrals and representations of the metaplectic group, preprint (1995).

[51] M. Tadić, Structure arising from induction and Jacquet modules of representations of classical p-adic groups, J. Algebra 177 (1995), no. 1, 1-33.

[52] M. Tadić, On reducibility of parabolic induction, Israel J. Math. 107 (1998), 29-91.

[53] J.-L. Waldspurger, Correspondances de Shimura et quaternions, Forum Math. 3 (1991), no. 3, $219-307$.

[54] N. R. Wallach, On the constant term of a square integrable automorphic form, Operator algebras and group representations, Vol. II (Neptun, 1980), 227-237, Monogr. Stud. Math., 18, Pitman, Boston, MA, 1984.

[55] M. H. Weissman, The Fourier-Jacobi map and small representations, Represent. Theory 7 (2003), 275299

[56] H. Xue, Refined global Gan-Gross-Prasad conjecture for Fourier-Jacobi periods on symplectic groups, Compos. Math. 153 (2017), no. 1, 68-131.

[57] H. Xue, Central values of degree six L-functions, J. Number Theory 203 (2019), 350-359.

[58] S. Yamana, Periods of automorphic forms: the trilinear case, J. Inst. Math. Jussieu 17 (2018), no. 1, 59-74.

[59] A. V. Zelevinsky, Induced representations of reductive $\mathfrak{p}$-adic groups. II. On irreducible representations of GL(n), Ann. Sci. École Norm. Sup. (4) 13 (1980), no. 2, 165-210.

Department of Mathematics, Hokkaido University, Kita 10, Nishi 8, Kita-Ku, Sapporo, Hokkaido, 060-0810, JAPAN

Email address: atobe@math.sci.hokudai.ac.jp 